\title{
Two Reliable Methods for Solving the $(3+1)$-Dimensional Space-Time Fractional Jimbo-Miwa Equation
}

\author{
Sekson Sirisubtawee, ${ }^{1,2}$ Sanoe Koonprasert, ${ }^{1,2}$ \\ Chaowanee Khaopant, ${ }^{1}$ and Wanassanun Porka ${ }^{1}$ \\ ${ }^{1}$ Department of Mathematics, Faculty of Applied Science, King Mongkut's University of Technology North Bangkok, \\ Bangkok 10800, Thailand \\ ${ }^{2}$ Centre of Excellence in Mathematics, CHE, Si Ayutthaya Road, Bangkok 10400, Thailand
}

Correspondence should be addressed to Sekson Sirisubtawee; sekson.s@sci.kmutnb.ac.th

Received 26 June 2017; Accepted 17 August 2017; Published 22 October 2017

Academic Editor: Kishin Sadarangani

Copyright (C) 2017 Sekson Sirisubtawee et al. This is an open access article distributed under the Creative Commons Attribution License, which permits unrestricted use, distribution, and reproduction in any medium, provided the original work is properly cited.

\begin{abstract}
We investigate methods for obtaining exact solutions of the $(3+1)$-dimensional nonlinear space-time fractional Jimbo-Miwa equation in the sense of the modified Riemann-Liouville derivative. The methods employed to analytically solve the equation are the $\left(G^{\prime} / G, 1 / G\right)$-expansion method and the novel $\left(G^{\prime} / G\right)$-expansion method. To the best of our knowledge, there are no researchers who have applied these methods to obtain exact solutions of the equation. The application of the methods is simple, elegant, efficient, and trustworthy. In particular, applying the novel $\left(G^{\prime} / G\right)$-expansion method to the equation, we obtain more exact solutions than using other existing methods such as the $\left(G^{\prime} / G\right)$-expansion method and the $\exp (-\Phi(\xi))$-expansion method. The exact solutions of the equation, obtained using the two methods, can be categorized in terms of hyperbolic, trigonometric, and rational functions. Some of the results obtained by the two methods are new and reported here for the first time. In addition, the obtained exact explicit solutions of the equation characterize many physical meanings such as soliton solitary wave solutions, periodic wave solutions, and singular multiple-soliton solutions.
\end{abstract}

\section{Introduction}

Various phenomena such as shallow water waves and multicellular biological dynamics arising in the nonlinear physical sciences [1,2], engineering [3, 4], and biology [5] can be modeled by a class of integrable nonlinear evolution equations which can be expressed in terms of nonlinear partial differential equations (NPDEs) of integer orders. Consequently, study of traveling wave solutions of NPDEs plays a significant role in the investigation of behaviors of nonlinear phenomena. Due to the efficiency, reliability, and easy use of symbolic software packages such as Maple or Mathematica, many powerful methods have been constructed and developed to analytically solve NPDEs with their aid. Over the last few decades, exact solutions, analytical approximate solutions, and numerical solutions of NPDEs have been successfully obtained. The methods for obtaining exact explicit solutions of NPDEs are, for example, the $\left(G^{\prime} / G\right)$-expansion method [6], the tanhfunction method $[7,8]$, the exp-function method $[9,10]$, the $F$-expansion method [11], Hirota's direct method $[12,13]$, Kudryashov method [14, 15], and so on. Examples of the methods for obtaining analytical approximate solutions to NPDEs are the variational iteration method $[16,17]$ (VIM), the Adomian decomposition method $[18,19](\mathrm{ADM})$, and the homotopy perturbation method [20, 21] (HPM). In addition, the examples of useful methods for solving NPDEs numerically are the generalized finite difference method [22], the finite volume method [23], and the finite element method [24].

Since fractional derivatives [25] such as the RiemannLiouville derivative and the Caputo derivative can describe the memory and hereditary properties of materials and processes which is different from ordinary derivatives, fractional differential equations (FDEs), which are associated with 
fractional derivatives and the generalization of the classical differential equations of integer orders, are expansively used to model various complex phenomena in many study fields such as physics [26], engineering [27], finance [28], and biology [29]. It has been found that the above-mentioned methods with their improvements (see, e.g., [30-33]) are also widely applicable to solve FDEs. Searching for exact explicit solutions to nonlinear fractional partial differential equations (NFPDEs) is a research field of active interest. Nowadays, many approaches with the help of symbolic software packages have been developed to efficiently provide exact solutions of NFPDEs, for example, the improved extended tanhcoth method [34], the improved generalized exp-function method [35], the fractional Riccati expansion method [36], the $\left(G^{\prime} / G, 1 / G\right)$-expansion method [37-41], and the novel $\left(G^{\prime} / G\right)$-expansion method [42-45]. The common idea of these mentioned methods is based on the homogeneous balance principle.

The $(3+1)$-dimensional Jimbo-Miwa equation, which was introduced by Jimbo and Miwa [46], is written as

$$
2 u_{y t}+3 u_{y} u_{x x}+3 u_{x} u_{x y}-3 u_{x z}+u_{x x x y}=0,
$$

which is the second member of integrable systems of the wellknown Kadomtsev-Petviashvili (KP) hierarchy [47, 48]. The Jimbo-Miwa equation in (1) is employed to describe particular interesting $(3+1)$-dimensional traveling waves in physics. According to the valuable literature obtaining exact solutions of (1) by several methods (see, e.g., [49-52]), the equation has a variety of solutions with distinct structures such as single-soliton solutions, multiple-soliton solutions, periodic wave solutions, and traveling wave solutions. The aim of this article is to apply the $\left(G^{\prime} / G, 1 / G\right)$-expansion method and the novel $\left(G^{\prime} / G\right)$-expansion method to solve the $(3+1)$ dimensional space-time fractional Jimbo-Miwa equation in the sense of Jumarie's modified Riemann-Liouville derivative. To the best of our knowledge, there are no researchers who have applied these methods to the fractional Jimbo-Miwa equation to obtain exact solutions. Some new exact solutions of the equation are reported for the first time. The rest of this paper is organized as follows. In Section 2, the definition of Jumarie's modified Riemann-Liouville derivative and some of its properties are given. Additionally, the descriptions of the $\left(G^{\prime} / G, 1 / G\right)$ and the novel $\left(G^{\prime} / G\right)$-expansion methods are provided. In Section 3, we illustrate the application of the two methods to the $(3+1)$-dimensional space-time fractional Jimbo-Miwa equation. In Section 4, we provide graphs and physical explanations of some selected exact solutions of the equation obtained by the two methods. Some conclusions and discussions which are relevant to the obtained results using the mentioned methods are given in Section 5.

\section{Mathematical Preliminaries}

In this section, we will provide fundamental concepts required in this paper for obtaining exact explicit solutions of the $(3+1)$-dimensional space-time fractional Jimbo-Miwa equation using the $\left(G^{\prime} / G, 1 / G\right)$-expansion method and the novel $\left(G^{\prime} / G\right)$-expansion method. We first give a definition and vital properties of the modified RiemannLiouville derivative defined by Jumarie. Then we describe the $\left(G^{\prime} / G, 1 / G\right)$-expansion method and the novel $\left(G^{\prime} / G\right)$ expansion method to obtain exact analytical solutions of nonlinear FDEs associated with the mentioned fractional derivative.

2.1. Jumarie's Modified Riemann-Liouville Derivative and Its Properties. The definition of the modified Riemann-Liouville derivative and its properties, which will be employed in this papers, are given as follows.

Definition 1. Assume that $f: \mathbb{R} \rightarrow \mathbb{R}, t \rightarrow f(t)$ denote a continuous (but not necessarily first-order differentiable) function. Jumarie's modified Riemann-Liouville derivative of order $\alpha$ is defined by the following expression $[53,54]$ :

$$
D_{t}^{\alpha} f(t)= \begin{cases}\frac{1}{\Gamma(-\alpha)} \int_{0}^{t}(t-\xi)^{-\alpha-1} f(\xi) d \xi, & \alpha<0, \\ \frac{1}{\Gamma(1-\alpha)} \frac{d}{d t} \int_{0}^{t}(t-\xi)^{-\alpha}[f(\xi)-f(0)] d \xi, & 0<\alpha<1, \\ \left(f^{(\alpha-n)}(t)\right)^{(n)}, & n \leq \alpha<n+1, n \geq 1,\end{cases}
$$

in which $\Gamma(\cdot)$ is the gamma function defined by

$$
\Gamma(\alpha)=\lim _{n \rightarrow \infty} \frac{n ! n^{\alpha}}{\alpha(\alpha+1)(\alpha+2) \cdots(\alpha+n)} .
$$

Some important properties of Jumarie's modified Riemann-Liouville derivative of order $\alpha$ are summarized as follows:

$$
\begin{aligned}
D_{t}^{\alpha} c=0, \quad \text { where } c \text { is a constant, } \\
D_{t}^{\alpha} t^{\gamma}=\frac{\Gamma(\gamma+1)}{\Gamma(\gamma+1-\alpha)} t^{\gamma-\alpha}, \quad \gamma>0,
\end{aligned}
$$

$$
\begin{aligned}
D_{t}^{\alpha}(c f(t)) & =c D_{t}^{\alpha} f(t), \\
D_{t}^{\alpha}(f(t) g(t)) & =g(t) D_{t}^{\alpha} f(t)+f(t) D_{t}^{\alpha} g(t), \\
D_{t}^{\alpha} f(g(t)) & =f_{g}^{\prime}(g(t)) D_{t}^{\alpha} g(t), \\
D_{t}^{\alpha} f(g(t)) & =D_{g}^{\alpha} f(g(t))\left(g_{t}^{\prime}\right)^{\alpha} .
\end{aligned}
$$

Remark 2. Properties (7)-(9) are direct results [53-55] from using the fractional Leibniz rule, the fractional Barrow's formula, and the relation $D_{t}^{\alpha} f(t) \cong \Gamma(\alpha+1) D_{t} f(t)$. Property 
(7) requires only that the functions $f$ and $g$ are continuous (not necessarily differentiable) functions. The use of property (8) requires that the function $f$ is differentiable with respect to the function $g$ and the function $g$ is continuous (not necessarily differentiable). On the other hand, property (9) needs the function $f$ to be continuous (not necessarily differentiable) with respect to the function $g$ and the function $g$ differentiable with respect to $t$. In particular, property (8) will be utilized in our work.

2.2. Descriptions of the $\left(G^{\prime} / G, 1 / G\right)$-Expansion Method and the Novel $\left(G^{\prime} / G\right)$-Expansion Method. In this section, the descriptions of the $\left(G^{\prime} / G, 1 / G\right)$-expansion method and the novel $\left(G^{\prime} / G\right)$-expansion method are concisely given. Consider a nonlinear fractional evolution partial differential equation in four independent variables $x, y, z$, and $t$ as follows:

$$
\begin{gathered}
F\left(u, D_{t}^{\alpha} u, D_{x}^{\eta} u, D_{y}^{\beta} u, D_{z}^{\gamma} u, D_{t}^{\alpha} D_{t}^{\alpha} u, D_{t}^{\alpha} D_{x}^{\eta} u, D_{t}^{\alpha} D_{y}^{\beta} u\right. \\
\left.D_{t}^{\alpha} D_{z}^{\gamma} u, D_{x}^{\eta} D_{y}^{\beta} u, \ldots\right)=0, \quad 0<\alpha, \eta, \beta, \gamma<1
\end{gathered}
$$

where $D_{t}^{\alpha} u, D_{x}^{\eta} u, D_{y}^{\beta} u$, and $D_{z}^{\gamma} u$ are Jumarie's modified Riemann-Liouville derivatives of a dependent variable $u$ with respect to $t, x, y$, and $z$. $F$ is a polynomial of the unknown function $u=u(x, y, z, t)$ and its various partial derivatives in which the highest order derivatives and nonlinear terms are involved. The first common step of the two methods is to convert the NFPDE in (10) into an ordinary differential equation (ODE) with a fractional complex transformation [56-58] by using a traveling wave variable $\xi$. We suppose that

$$
\begin{aligned}
U(\xi) & =u(x, y, z, t), \\
\xi & =\frac{x^{\eta}}{\Gamma(1+\eta)}+\frac{y^{\beta}}{\Gamma(1+\beta)}+\frac{z^{\gamma}}{\Gamma(1+\gamma)}-\frac{V t^{\alpha}}{\Gamma(1+\alpha)},
\end{aligned}
$$

where $V$ is a nonzero arbitrary constant. With the transformation in (11) and an integration with respect to $\xi$ (if possible), (10) is reduced to an ODE for $U=U(\xi)$ as follows:

$$
P\left(U, U^{\prime}, U^{\prime \prime}, U^{\prime \prime \prime}, \ldots\right)=0
$$

where $P$ is a function of $U(\xi)$ and its various derivatives. The prime notation $\left(^{\prime}\right)$ denotes the derivative with respect to $\xi$. Next we provide the main steps of the $\left(G^{\prime} / G, 1 / G\right)$-expansion method and then the novel $\left(G^{\prime} / G\right)$-expansion method for solving the ODE in (12) as demonstrated below.

2.2.1. The $\left(G^{\prime} / G, 1 / G\right)$-Expansion Method. The following relevant concepts required before providing the main steps of the $\left(G^{\prime} / G, 1 / G\right)$-expansion method are introduced below. Consider the following second-order linear ODE:

$$
G^{\prime \prime}(\xi)+\lambda G(\xi)=\mu,
$$

where the prime notation $\left(^{\prime}\right)$ denotes the derivative with respect to $\xi$ and where $\lambda, \mu$ are constants. Next we set

$$
\begin{gathered}
\phi(\xi)=\frac{G^{\prime}(\xi)}{G(\xi)}, \\
\psi(\xi)=\frac{1}{G(\xi)} .
\end{gathered}
$$

Equations (13) and (14) can be transformed into the system of two nonlinear ordinary differential equations as follows:

$$
\begin{aligned}
& \phi^{\prime}=-\phi^{2}+\mu \psi-\lambda, \\
& \psi^{\prime}=-\phi \psi .
\end{aligned}
$$

The solutions of (13) can be categorized into the following three cases.

Case 1. If $\lambda<0$, then the general solution of (13) is of the form

$$
G(\xi)=A_{1} \sinh (\xi \sqrt{-\lambda})+A_{2} \cosh (\xi \sqrt{-\lambda})+\frac{\mu}{\lambda},
$$

and we have

$$
\psi^{2}=\frac{-\lambda}{\lambda^{2} \sigma_{1}+\mu^{2}}\left(\phi^{2}-2 \mu \psi+\lambda\right),
$$

where $A_{1}$ and $A_{2}$ are arbitrary constants and $\sigma_{1}=A_{1}^{2}-A_{2}^{2}$.

Case 2. If $\lambda>0$, then the general solution of (13) can be given as

$$
G(\xi)=A_{1} \sin (\xi \sqrt{\lambda})+A_{2} \cos (\xi \sqrt{\lambda})+\frac{\mu}{\lambda},
$$

and we have the following associated relation:

$$
\psi^{2}=\frac{\lambda}{\lambda^{2} \sigma_{2}-\mu^{2}}\left(\phi^{2}-2 \mu \psi+\lambda\right),
$$

where $A_{1}$ and $A_{2}$ are arbitrary constants and $\sigma_{2}=A_{1}^{2}+A_{2}^{2}$.

Case 3. If $\lambda=0$, then the general solution of (13) can be written as

$$
G(\xi)=\frac{\mu}{2} \xi^{2}+A_{1} \xi+A_{2}
$$

and the corresponding relation is

$$
\psi^{2}=\frac{1}{A_{1}^{2}-2 \mu A_{2}}\left(\phi^{2}-2 \mu \psi\right),
$$

where $A_{1}$ and $A_{2}$ are arbitrary constants.

The main steps of the $\left(G^{\prime} / G, 1 / G\right)$-expansion method [37-41] are as follows.

Step 1. Suppose that the solution to (12) can be expressed by a polynomial in the two variables $\phi$ and $\psi$ as follows:

$$
U(\xi)=a_{0}+\sum_{j=1}^{N} a_{j} \phi^{j}+\sum_{j=1}^{N} b_{j} \phi^{j-1} \psi,
$$


where $a_{0}, a_{j}$, and $b_{j}(j=1,2, \ldots, N)$ are constants to be determined later with $a_{N}^{2}+b_{N}^{2} \neq 0$ and where the functions $\phi=\phi(\xi)$ and $\psi=\psi(\xi)$ are implicitly associated with (13) using the relations in (14).

Step 2. Determine the positive integer $N$ in (22) by inserting (22) into (12) and then using the homogeneous balance between the highest order derivatives and the nonlinear terms in (12). If the degree of $U(\xi)$ is $\operatorname{Deg}[U(\xi)]=N$, then the degree of other terms will be expressed as follows:

$$
\begin{gathered}
\operatorname{Deg}\left[\frac{d^{q} U(\xi)}{d \xi^{q}}\right]=N+q \\
\operatorname{Deg}\left[(U(\xi))^{p}\left(\frac{d^{q} U(\xi)}{d \xi^{q}}\right)^{s}\right]=N p+s(N+q) .
\end{gathered}
$$

In particular, if the balance number $N$ of some nonlinear equations is not a positive integer then the special transformations (e.g., when $N=q / p$ is a fraction in the lowest terms, we set $\left.U(\xi)=W^{q / p}(\xi)\right)$ are applied for $U(\xi)$ in (12) to have a new equation in terms of the new function $W(\xi)$ with a positive integer balance number (see details in $[39,59]$ ).

Step 3. Substituting the resulting equation of (22) into (12) with the aid of (15) and (17), the function $P$ in (12) can be converted into a polynomial in $\phi$ and $\psi$, in which the degree of $\psi$ is not larger than one. Equating each coefficients of the resulting polynomial to zero, we obtain a system of algebraic equations, which can be solved using the symbolic computational packages such as Maple or Mathematica, for the following unknowns $a_{0}, a_{j}, b_{j}(j=1,2, \ldots, N), V, \mu$, $\lambda(<0), A_{1}$, and $A_{2}$. The resulting traveling wave solutions generated by this step with the transformation in (11) are expressed by hyperbolic functions.

Step 4. In the same manner as Step 3, substituting the resulting equation of (22) into (12) with the aid of (15) and (19) for $\lambda>0$, we can obtain the exact solutions of (10) by using the transformation in (11). The obtained exact solutions are written as trigonometric functions.

Step 5. In the same manner as Step 3, substituting the resulting equation of (22) into (12) with the aid of (15) and (21) for $\lambda=0$, we can obtain the traveling wave solutions of (10) by using the transformation in (11). The resulting exact solutions are expressed by rational functions.

2.2.2. The Novel $\left(G^{\prime} / G\right)$-Expansion Method. In the following, we give the main steps of the novel $\left(G^{\prime} / G\right)$-expansion method [42-45].

Step 1. Suppose that the solution of (12) can be expressed in power of $\psi(\xi)$ as follows:

$$
U(\xi)=\sum_{j=-N}^{N} a_{j}(\psi(\xi))^{j},
$$

where

$$
\psi(\xi)=d+\phi(\xi) \quad \text { with } \phi(\xi)=\frac{G^{\prime}(\xi)}{G(\xi)} .
$$

The unknown constants $a_{-N}$ or $a_{N}$ may be zero, but both of them cannot be zero simultaneously. The constants $a_{j}(j=$ $0, \pm 1, \pm 2, \ldots, \pm N)$ and $d$ are determined at a later step and the function $G=G(\xi)$ satisfies the following nonlinear secondorder ODE:

$$
G G^{\prime \prime}=\lambda G G^{\prime}+\mu G^{2}+v\left(G^{\prime}\right)^{2}
$$

where the prime notation $\left(^{\prime}\right)$ denotes the derivative with respect to $\xi$ and where $\lambda, \mu$, and $v$ are real parameters.

The Cole-Hopf transformation $\phi(\xi)=\ln (G(\xi))_{\xi}=$ $G^{\prime}(\xi) / G(\xi)$ reduces $(26)$ into the following generalized Riccati equation:

$$
\phi^{\prime}(\xi)=\mu+\lambda \phi(\xi)+(v-1) \phi^{2}(\xi) .
$$

It is been discovered that (27) has thirty-nine solutions (see $[60,61]$ and Appendix for details).

Step 2. The value of the positive integer $N$ can be computed by balancing the highest order linear terms with nonlinear terms of the highest order occurring in (12). If the degree of $U(\xi)$ is $\operatorname{Deg}[U(\xi)]=N$, then the degree of the other expressions will be expressed as in (23).

Step 3. Substituting (24) along with (25) and (26) into (12), we obtain polynomials in $(d+\phi(\xi))^{k}(k=0, \pm 1, \pm 2, \ldots, \pm M$ where $M$ is a positive integer). Collecting all coefficients of like-power of the resulting polynomials to zero, we yield an overdetermined set of algebraic equations for $a_{j}(j=$ $0, \pm 1, \pm 2, \ldots, \pm N), d$, and $V$.

Step 4. Assuming that the unknown constants of the algebraic equations in Step 3 can be obtained, we substitute the values of the constants together with the solutions of (27) into (24) to obtain exact traveling wave solutions of the nonlinear fractional evolution partial differential equation (10) when $\xi$ is set in (11).

Remark 3. It is worth noting that the novel $\left(G^{\prime} / G\right)$-expansion method is a generalization of many types of $\left(G^{\prime} / G\right)$ expansion methods. First, if we set $d=0$ in (25) and $v=0$ in (26) and negative indices of $\left(G^{\prime} / G\right)$ are zero in (24), then the method is reduced to the basic $\left(G^{\prime} / G\right)$-expansion methods proposed by Wang et al. [62]. Secondly, if $d=0$ in (25) and $v=0$ in (26) then the method is identical to the improved $\left(G^{\prime} / G\right)$-expansion methods introduced by Zhang et al. [63]. Thirdly, if $\lambda, \mu$, and $v$ in (26) are replaced by $-\lambda,-\mu$, and 0 , respectively, then the novel $\left(G^{\prime} / G\right)$-expansion method translates to the generalized and improved $\left(G^{\prime} / G\right)$-expansion method presented by Akbar et al. [64]. Finally, if we let $a_{j}(j=$ $0,1,2, \ldots, N)$ be functions of $x$ and $t$ instead of constants and $v=0$ in (26) then the method coincides with the generalized $\left(G^{\prime} / G\right)$-expansion method introduced by Zhang et al. [65]. 


\section{Application of the Two Methods to the $(3+1)$-Dimensional Space-Time Fractional Jimbo-Miwa Equation}

Consider the $(3+1)$-dimensional space-time fractional Jimbo-Miwa equation [66]

$$
\begin{gathered}
2 D_{y}^{\beta} D_{t}^{\alpha} u+3 D_{y}^{\beta} u D_{x}^{2 \eta} u+3 D_{x}^{\eta} u D_{x}^{\eta} D_{y}^{\beta} u-3 D_{x}^{\eta} D_{z}^{\gamma} u \\
+D_{x}^{3 \eta} D_{y}^{\beta} u=0, \quad 0<\eta, \beta, \gamma, \alpha \leq 1
\end{gathered}
$$

where $D_{s}^{\kappa} u$ denotes Jumarie's modified Riemann-Liouville derivative of a dependent variable $u$ with respect to $s$ with the fractional order $\kappa$ and $u(x, y, z, t)$ is a traveling wave solution of (28) which is an unknown function of four independent variables $x, y, z$, and $t$. Using the traveling wave transformation in second equation of (11) and the properties in (5) and (8), we obtain $D_{t}^{\alpha} U(\xi)=-V U^{\prime}$, $D_{x}^{\eta} U(\xi)=U^{\prime}, D_{y}^{\beta} U(\xi)=U^{\prime}, D_{z}^{\gamma} U(\xi)=U^{\prime}, D_{y}^{\beta} D_{t}^{\alpha} U(\xi)=$ $-V U^{\prime \prime}, D_{x}^{2 \eta} U(\xi)=D_{x}^{\eta}\left(D_{x}^{\eta} U(\xi)\right)=U^{\prime \prime}, D_{x}^{\eta} D_{y}^{\beta} U(\xi)=$ $D_{x}^{\eta}\left(U^{\prime}(\xi)\right)=U^{\prime \prime}, D_{x}^{\eta} D_{z}^{\gamma} U(\xi)=D_{x}^{\eta}\left(U^{\prime}(\xi)\right)=U^{\prime \prime}$, and $D_{x}^{3 \eta} D_{y}^{\beta} U(\xi)=D_{x}^{3 \eta}\left(U^{\prime}(\xi)\right)=D_{x}^{2 \eta}\left(D_{x}^{\eta}\left(U^{\prime}(\xi)\right)\right)=$ $D_{x}^{2 \eta}\left(U^{\prime \prime}(\xi)\right)=D_{x}^{\eta}\left(D_{x}^{\eta}\left(U^{\prime \prime}(\xi)\right)\right)=D_{x}^{\eta}\left(U^{\prime \prime \prime}(\xi)\right)=U^{(4)}$. Hence, (28) is reduced into the ODE of the variable $U=U(\xi)$ as

$$
-(2 V+3) U^{\prime \prime}+6 U^{\prime} U^{\prime \prime}+U^{(4)}=0
$$

where the prime notation $\left(^{\prime}\right)$ denotes the derivative with respect to $\xi$. Integrating (29) with respect to $\xi$ and then choosing the constant of integration to be zero, we obtain the following ODE:

$$
(2 V+3) U^{\prime}-3\left(U^{\prime}\right)^{2}-U^{\prime \prime \prime}=0 .
$$

Applying the formulas in (23) for balancing the highest order degree terms in $U^{\prime \prime \prime}$ and $\left(U^{\prime}\right)^{2}$ in (30), we have $N+3=$ $2(N+1) \Rightarrow N=1$. Consequently, the solution forms of (30) obtained using the $\left(G^{\prime} / G, 1 / G\right)$-expansion method and the novel $\left(G^{\prime} / G\right)$-expansion method are formed with $N=1$ by using (22) and (24), respectively. Next, we will apply the mentioned two methods to (30) to eventually produce exact solutions of the fractional Jimbo-Miwa equation in (28) as follows.

3.1. Using the $\left(G^{\prime} / G, 1 / G\right)$-Expansion Method. From the balance which is done above, (22) is reduced to

$$
U(\xi)=a_{0}+a_{1} \phi(\xi)+b_{1} \psi(\xi),
$$

where the constant coefficients $a_{0}, a_{1}$, and $b_{1}$ are determined at a later step, subject to the inequality $a_{1}^{2}+b_{1}^{2} \neq 0$. There are three cases of the function $G(\xi)$ associated with the functions $\phi(\xi)$ and $\psi(\xi)$ of the solution in (31) depending on the sign of $\lambda$ described above.

Case 1 (hyperbolic function solutions $(\lambda<0)$ ). If $\lambda<0$, we substitute (31) into (30) along with the use of (15) and (17). Then the left-hand side of (30) turns out to be a polynomial in $\phi(\xi)$ and $\psi(\xi)$. Setting all of the coefficients of this resulting polynomial to be zero, we obtain the following system of nonlinear algebraic equations in $a_{0}, a_{1}, b_{1}, V, \mu, \lambda, A_{1}, A_{2}$ :

$$
\begin{aligned}
& \phi^{4}(\xi):-3 \lambda^{2} A_{1}^{2} a_{1}^{2}+3 \lambda^{2} A_{2}^{2} a_{1}^{2}+6 \lambda^{2} A_{1}^{2} a_{1} \\
& -6 \lambda^{2} A_{2}^{2} a_{1}-3 \mu^{2} a_{1}^{2}+6 \mu^{2} a_{1}+3 \lambda b_{1}^{2}=0, \\
& \phi^{3}(\xi):-6 \lambda \mu a_{1} b_{1}+6 \lambda \mu b_{1}=0, \\
& \phi^{3}(\xi) \psi(\xi):-6 \lambda^{2} A_{1}^{2} a_{1} b_{1}+6 \lambda^{2} A_{2}^{2} a_{1} b_{1}+6 \lambda^{2} A_{1}^{2} b_{1} \\
& -6 \lambda^{2} A_{2}^{2} b_{1}-6 \mu^{2} a_{1} b_{1}+6 \mu^{2} b_{1}=0, \\
& \phi^{2}(\xi):-6 \lambda^{3} A_{1}^{2} a_{1}^{2}+6 \lambda^{3} A_{2}^{2} a_{1}^{2}-2 V \lambda^{2} A_{1}^{2} a_{1} \\
& +2 V \lambda^{2} A_{2}^{2} a_{1}+8 \lambda^{3} A_{1}^{2} a_{1}-8 \lambda^{3} A_{2}^{2} a_{1}-3 \lambda \mu^{2} a_{1}^{2} \\
& -3 \lambda^{2} A_{1}^{2} a_{1}+3 \lambda^{2} A_{2}^{2} a_{1}-2 V \mu^{2} a_{1}+5 \lambda \mu^{2} a_{1} \\
& +3 \lambda^{2} b_{1}^{2}-3 \mu^{2} a_{1}=0, \\
& \phi^{2}(\xi) \psi(\xi): 6 \lambda^{2} \mu A_{1}^{2} a_{1}^{2}-6 \lambda^{2} \mu A_{2}^{2} a_{1}^{2}-12 \lambda^{2} \mu A_{1}^{2} a_{1} \\
& +12 \lambda^{2} \mu A_{2}^{2} a_{1}+6 \mu^{3} a_{1}^{2}-12 \mu^{3} a_{1}-6 \lambda \mu b_{1}^{2}=0, \\
& \phi(\xi):-6 \lambda^{2} \mu a_{1} b_{1}+6 \lambda^{2} \mu b_{1}=0, \\
& \phi(\xi) \psi(\xi):-6 \lambda^{3} A_{1}^{2} a_{1} b_{1}+6 \lambda^{3} A_{2}^{2} a_{1} b_{1}-2 V \lambda^{2} A_{1}^{2} b_{1} \\
& +2 V \lambda^{2} A_{2}^{2} b_{1}+5 \lambda^{3} A_{1}^{2} b_{1}-5 \lambda^{3} A_{2}^{2} b_{1}+6 \lambda \mu^{2} a_{1} b_{1} \\
& -3 \lambda^{2} A_{1}^{2} b_{1}+3 \lambda^{2} A_{2}^{2} b_{1}-2 V \mu^{2} b_{1}-7 \lambda \mu^{2} b_{1} \\
& -3 \mu^{2} b_{1}=0 \text {, } \\
& \psi(\xi): 6 \lambda^{3} \mu A_{1}^{2} a_{1}^{2}-6 \lambda^{3} \mu A_{2}^{2} a_{1}^{2}+2 V \lambda^{2} \mu A_{1}^{2} a_{1} \\
& -2 V \lambda^{2} \mu A_{2}^{2} a_{1}-5 \lambda^{3} \mu A_{1}^{2} a_{1}+5 \lambda^{3} \mu A_{2}^{2} a_{1} \\
& +3 \lambda^{2} \mu A_{1}^{2} a_{1}-3 \lambda^{2} \mu A_{2}^{2} a_{1}+2 V \mu^{3} a_{1}+\lambda \mu^{3} a_{1} \\
& +3 \mu^{3} a_{1}=0 \text {, } \\
& \phi^{0}(\xi):-3 \lambda^{4} A_{1}^{2} a_{1}^{2}+3 \lambda^{4} A_{2}^{2} a_{1}^{2}-2 V \lambda^{3} A_{1}^{2} a_{1} \\
& +2 V \lambda^{3} A_{2}^{2} a_{1}+2 \lambda^{4} A_{1}^{2} a_{1}-2 \lambda^{4} A_{2}^{2} a_{1}-3 \lambda^{3} A_{1}^{2} a_{1} \\
& +3 \lambda^{3} A_{2}^{2} a_{1}-2 V \lambda \mu^{2} a_{1}-\lambda^{2} \mu^{2} a_{1}-3 \lambda \mu^{2} a_{1}=0 .
\end{aligned}
$$

It is required that the denominator of $\psi^{2}$ in (17) is not zero, so system (32) is valid if $\lambda^{2}\left(A_{1}^{2}-A_{2}^{2}\right)+\mu^{2} \neq 0$. By solving the above algebraic system using the Maple package program, we have the following results.

Result 1

$$
\begin{gathered}
a_{1}=2, \\
b_{1}=0, \\
V=-2 \lambda-\frac{3}{2},
\end{gathered}
$$




$$
\mu=0 \text {, }
$$

$a_{0}, \lambda(<0)$ are arbitrary constants.

From (16), (31), and (33), we obtain the traveling wave solution of (28) as follows:

$$
\begin{aligned}
& u_{1}^{1}(x, y, z, t)=a_{0} \\
& +\frac{2\left(A_{1} \cosh (\xi \sqrt{-\lambda}) \sqrt{-\lambda}+A_{2} \sinh (\xi \sqrt{-\lambda}) \sqrt{-\lambda}\right)}{A_{1} \sinh (\xi \sqrt{-\lambda})+A_{2} \cosh (\xi \sqrt{-\lambda})},
\end{aligned}
$$

where $\xi$ is defined in (11) with $V$ shown in (33) and $A_{1}, A_{2}$ are arbitrary constants.

Result 2

$$
\begin{gathered}
a_{1}=1, \\
b_{1}= \pm \sqrt{-\frac{\lambda^{2} A_{1}^{2}-\lambda^{2} A_{2}^{2}+\mu^{2}}{\lambda}} \\
V=-\frac{\lambda}{2}-\frac{3}{2}
\end{gathered}
$$

$a_{0}, \mu, \lambda(<0)$ are arbitrary constants.

From (16), (31), and (35), we obtain the traveling wave solution of (28) as follows:

$$
\begin{aligned}
& u_{2}^{1}(x, y, z, t) \\
& =a_{0} \\
& \quad+\frac{A_{1} \cosh (\xi \sqrt{-\lambda}) \sqrt{-\lambda}+A_{2} \sinh (\xi \sqrt{-\lambda}) \sqrt{-\lambda}}{A_{1} \sinh (\xi \sqrt{-\lambda})+A_{2} \cosh (\xi \sqrt{-\lambda})+\mu / \lambda} \\
& \quad \pm \frac{\sqrt{-\left(\lambda^{2} A_{1}^{2}-\lambda^{2} A_{2}^{2}+\mu^{2}\right) / \lambda}}{A_{1} \sinh (\xi \sqrt{-\lambda})+A_{2} \cosh (\xi \sqrt{-\lambda})+\mu / \lambda}
\end{aligned}
$$

where $\xi$ is defined in (11) with $V$ shown in (35) and $A_{1}, A_{2}$ are arbitrary constants.

Case 2 (trigonometric function solutions $(\lambda>0)$ ). If $\lambda>0$, we substitute (31) into (30) along with the use of (15) and (19). Then the left-hand side of (30) becomes a polynomial in $\phi(\xi)$ and $\psi(\xi)$. Setting all of the coefficients of the resulting polynomial to be zero, we obtain the following system of nonlinear algebraic equations in $a_{0}, a_{1}, b_{1}, V, \mu, \lambda, A_{1}, A_{2}$ :

$$
\begin{aligned}
& \phi^{4}(\xi):-3 \lambda^{2} A_{1}^{2} a_{1}^{2}-3 \lambda^{2} A_{2}^{2} a_{1}^{2}+6 \lambda^{2} A_{1}^{2} a_{1} \\
& \quad+6 \lambda^{2} A_{2}^{2} a_{1}+3 \mu^{2} a_{1}^{2}-3 \lambda b_{1}^{2}-6 \mu^{2} a_{1}=0, \\
& \phi^{3}(\xi): 6 \lambda \mu a_{1} b_{1}-6 \lambda \mu b_{1}=0, \\
& \phi^{3}(\xi) \psi(\xi):-6 \lambda^{2} A_{1}^{2} a_{1} b_{1}-6 \lambda^{2} A_{2}^{2} a_{1} b_{1}+6 \lambda^{2} A_{1}^{2} b_{1} \\
& \quad+6 \lambda^{2} A_{2}^{2} b_{1}+6 \mu^{2} a_{1} b_{1}-6 \mu^{2} b_{1}=0,
\end{aligned}
$$

$$
\begin{aligned}
& \phi^{2}(\xi):-6 \lambda^{3} A_{1}^{2} a_{1}^{2}-6 \lambda^{3} A_{2}^{2} a_{1}^{2}-2 V \lambda^{2} A_{1}^{2} a_{1} \\
& -2 V \lambda^{2} A_{2}^{2} a_{1}+8 \lambda^{3} A_{1}^{2} a_{1}+8 \lambda^{3} A_{2}^{2} a_{1}-3 \lambda^{2} A_{1}^{2} a_{1} \\
& -3 \lambda^{2} A_{2}^{2} a_{1}+3 \lambda \mu^{2} a_{1}^{2}+2 V \mu^{2} a_{1}-3 \lambda^{2} b_{1}^{2}-5 \lambda \mu^{2} a_{1} \\
& +3 \mu^{2} a_{1}=0 \text {, } \\
& \phi^{2}(\xi) \psi(\xi): 6 \lambda^{2} \mu A_{1}^{2} a_{1}^{2}+6 \lambda^{2} \mu A_{2}^{2} a_{1}^{2}-12 \lambda^{2} \mu A_{1}^{2} a_{1} \\
& -12 \lambda^{2} \mu A_{2}^{2} a_{1}-6 \mu^{3} a_{1}^{2}+6 \lambda \mu b_{1}^{2}+12 \mu^{3} a_{1}=0, \\
& \phi(\xi): 6 \lambda^{2} \mu a_{1} b_{1}-6 \lambda^{2} \mu b_{1}=0, \\
& \phi(\xi) \psi(\xi):-6 \lambda^{3} A_{1}^{2} a_{1} b_{1}-6 \lambda^{3} A_{2}^{2} a_{1} b_{1}-2 V \lambda^{2} A_{1}^{2} b_{1} \\
& -2 V \lambda^{2} A_{2}^{2} b_{1}+5 \lambda^{3} A_{1}^{2} b_{1}+5 \lambda^{3} A_{2}^{2} b_{1}-3 \lambda^{2} A_{1}^{2} b_{1} \\
& -3 \lambda^{2} A_{2}^{2} b_{1}-6 \lambda \mu^{2} a_{1} b_{1}+2 V \mu^{2} b_{1}+7 \lambda \mu^{2} b_{1} \\
& +3 \mu^{2} b_{1}=0 \\
& \psi(\xi): 6 \lambda^{3} \mu A_{1}^{2} a_{1}^{2}+6 \lambda^{3} \mu A_{2}^{2} a_{1}^{2}+2 V \lambda^{2} \mu A_{1}^{2} a_{1} \\
& +2 V \lambda^{2} \mu A_{2}^{2} a_{1}-5 \lambda^{3} \mu A_{1}^{2} a_{1}-5 \lambda^{3} \mu A_{2}^{2} a_{1} \\
& +3 \lambda^{2} \mu A_{1}^{2} a_{1}+3 \lambda^{2} \mu A_{2}^{2} a_{1}-2 V \mu^{3} a_{1}-\lambda \mu^{3} a_{1} \\
& -3 \mu^{3} a_{1}=0, \\
& \phi^{0}(\xi):-3 \lambda^{4} A_{1}^{2} a_{1}^{2}-3 \lambda^{4} A_{2}^{2} a_{1}^{2}-2 V \lambda^{3} A_{1}^{2} a_{1} \\
& -2 V \lambda^{3} A_{2}^{2} a_{1}+2 \lambda^{4} A_{1}^{2} a_{1}+2 \lambda^{4} A_{2}^{2} a_{1}-3 \lambda^{3} A_{1}^{2} a_{1} \\
& -3 \lambda^{3} A_{2}^{2} a_{1}+2 V \lambda \mu^{2} a_{1}+\lambda^{2} \mu^{2} a_{1}+3 \lambda \mu^{2} a_{1}=0 \text {. }
\end{aligned}
$$

It is required that the denominator of $\psi^{2}$ in (19) is not zero; thus system (37) is solvable if $\lambda^{2}\left(A_{1}^{2}+A_{2}^{2}\right)-\mu^{2} \neq 0$. On solving the above algebraic system using the Maple package program, we obtain the following results.

Result 1

$$
\begin{gathered}
a_{1}=2, \\
b_{1}=0, \\
V=-2 \lambda-\frac{3}{2}, \\
\mu=0,
\end{gathered}
$$

$a_{0}, \lambda(>0)$ are arbitrary constants.

From (18), (31), and (38), we obtain the traveling wave solution of (28) as follows:

$$
\begin{aligned}
& u_{1}^{2}(x, y, z, t) \\
& \quad=a_{0}+\frac{2\left(A_{1} \cos (\xi \sqrt{\lambda}) \sqrt{\lambda}-A_{2} \sin (\xi \sqrt{\lambda}) \sqrt{\lambda}\right)}{A_{1} \sin (\xi \sqrt{\lambda})+A_{2} \cos (\xi \sqrt{\lambda})},
\end{aligned}
$$


where $\xi$ is defined in (11) with $V$ shown in (38) and $A_{1}, A_{2}$ are arbitrary constants.

Result 2

$$
\begin{gathered}
a_{1}=1, \\
b_{1}= \pm \sqrt{-\frac{-\lambda^{2} A_{1}^{2}-\lambda^{2} A_{2}^{2}+\mu^{2}}{\lambda}} \\
V=-\frac{\lambda}{2}-\frac{3}{2},
\end{gathered}
$$

$a_{0}, \mu, \lambda(>0)$ are arbitrary constants.

From (18), (31), and (40), we deduce the traveling wave solution of (28) as follows:

$$
\begin{aligned}
& u_{2}^{2}(x, y, z, t) \\
& =a_{0}+\frac{A_{1} \cos (\xi \sqrt{\lambda}) \sqrt{\lambda}-A_{2} \sin (\xi \sqrt{\lambda}) \sqrt{\lambda}}{A_{1} \sin (\xi \sqrt{\lambda})+A_{2} \cos (\xi \sqrt{\lambda})+\mu / \lambda} \\
& \pm \frac{\sqrt{-\left(-\lambda^{2} A_{1}^{2}-\lambda^{2} A_{2}^{2}+\mu^{2}\right) / \lambda}}{A_{1} \sin (\xi \sqrt{\lambda})+A_{2} \cos (\xi \sqrt{\lambda})+\mu / \lambda},
\end{aligned}
$$

where $\xi$ is defined in (11) with $V$ shown in (40) and $A_{1}, A_{2}$ are arbitrary constants.

Case 3 (rational function solutions $(\lambda=0)$ ). If $\lambda=0$, we substitute (31) into (30) along with the use of (15) and (21). Then the left-hand side of (30) becomes a polynomial in $\phi(\xi)$ and $\psi(\xi)$. Setting all of the coefficients of this polynomial to be zero, we obtain the following system of nonlinear algebraic equations in $a_{0}, a_{1}, b_{1}, V, \mu, A_{1}, A_{2}$ :

$$
\begin{aligned}
& \phi^{4}(\xi): 6 \mu A_{2} a_{1}^{2}-3 A_{1}^{2} a_{1}^{2}-12 \mu A_{2} a_{1}+6 A_{1}^{2} a_{1} \\
& \quad-3 b_{1}^{2}=0, \\
& \phi^{3}(\xi): 6 \mu a_{1} b_{1}-6 \mu b_{1}=0, \\
& \phi^{3}(\xi) \psi(\xi): 12 \mu A_{2} a_{1} b_{1}-6 A_{1}^{2} a_{1} b_{1}-12 \mu A_{2} b_{1} \\
& \quad+6 A_{1}^{2} b_{1}=0, \\
& \phi^{2}(\xi): 4 V \mu A_{2} a_{1}-2 V A_{1}^{2} a_{1}-3 \mu^{2} a_{1}^{2}+3 \mu^{2} a_{1} \\
& \quad+6 \mu A_{2} a_{1}-3 A_{1}^{2} a_{1}=0, \\
& \phi^{2}(\xi) \psi(\xi):-12 \mu^{2} A_{2} a_{1}^{2}+6 \mu A_{1}^{2} a_{1}^{2}+24 \mu^{2} A_{2} a_{1} \\
& \quad-12 \mu A_{1}^{2} a_{1}+6 \mu b_{1}^{2}=0, \\
& \phi(\xi) \psi(\xi): 4 V \mu A_{2} b_{1}-2 V A_{1}^{2} b_{1}-12 \mu^{2} a_{1} b_{1}+12 \mu^{2} b_{1} \\
& \quad+6 \mu A_{2} b_{1}-3 A_{1}^{2} b_{1}=0, \\
& \psi(\xi):-4 V \mu^{2} A_{2} a_{1}+2 V \mu A_{1}^{2} a_{1}+6 \mu^{3} a_{1}^{2}-6 \mu^{3} a_{1} \\
& \quad-6 \mu^{2} A_{2} a_{1}+3 \mu A_{1}^{2} a_{1}=0 .
\end{aligned}
$$

It is required that the denominator of $\psi^{2}$ in (21) is not zero; hence system (42) is valid if $A_{1}^{2}-2 \mu A_{2} \neq 0$. By solving the above algebraic system using the Maple package program, we display only the following interesting results.

Result 1

$$
\begin{gathered}
a_{1}=1, \\
b_{1}= \pm \sqrt{-2 \mu A_{2}+A_{1}^{2}} \\
V=-\frac{3}{2}
\end{gathered}
$$

$a_{0}, \mu$ are arbitrary constants.

From (20), (31), and (43), we obtain the traveling wave solution of (28) as follows:

$$
\begin{aligned}
u_{1}^{3}(x, y, z, t)= & a_{0}+\frac{\mu \xi+A_{1}}{(1 / 2) \mu \xi^{2}+A_{1} \xi+A_{2}} \\
& \pm \frac{\sqrt{-2 \mu A_{2}+A_{1}^{2}}}{(1 / 2) \mu \xi^{2}+A_{1} \xi+A_{2}},
\end{aligned}
$$

where $\xi$ is defined in (11) with $V$ shown in (43) and $A_{1}, A_{2}$ are arbitrary constants.

Result 2

$$
\begin{gathered}
a_{1}=1, \\
b_{1}=A_{1}, \\
V=-\frac{3}{2}, \\
\mu=0,
\end{gathered}
$$

$a_{0}$ is an arbitrary constant.

From (20), (31), and (45), we deduce the traveling wave solution of (28) as follows:

$$
u_{2}^{3}(x, y, z, t)=a_{0}+\frac{2 A_{1}}{A_{1} \xi+A_{2}},
$$

where $\xi$ is defined in (11) with $V$ shown in (45) and $A_{1}, A_{2}$ are arbitrary constants.

Aksoy et al. [67] utilized the $\left(G^{\prime} / G\right)$-expansion method to generate the exact solutions of (28), which are expressed in (33), (34), and (35) in their paper. We have found that our exact solutions in (34), (39), and (46), obtained by the $\left(G^{\prime} / G, 1 / G\right)$-expansion method, have the same mathematical structures as their results. While the solutions in (36), (41), and (44) do not appear in [67] and they are all new and not found elsewhere.

3.2. Using the Novel $\left(G^{\prime} / G\right)$-Expansion Method. Obtaining $N=1$ from balancing the highest order derivative $U^{\prime \prime \prime}$ with the nonlinear term of the highest order $\left(U^{\prime}\right)^{2}$, the solution of (30) using the novel $\left(G^{\prime} / G\right)$-expansion method has the following form:

$$
U(\xi)=a_{-1}(\psi(\xi))^{-1}+a_{0}+a_{1}(\psi(\xi)),
$$


where $\psi(\xi)=d+\phi(\xi)$ and $\phi(\xi)=G^{\prime} / G$. Substituting (47) into (30) and then performing algebraic manipulations, the lefthand side of (30) is converted into polynomials of $(\psi(\xi))^{-j}=$ $(d+\phi(\xi))^{-j}$ and $(\psi(\xi))^{j}=(d+\phi(\xi))^{j}$ where $j=0,1,2,3,4$.

Equating the coefficients of like-power of these polynomials to zero, we obtain the following set of nonlinear algebraic equations:

$$
\begin{aligned}
& \psi^{4}(\xi):-6 v^{3} a_{1}-3 v^{2} a_{1}^{2}+18 v^{2} a_{1}+6 v a_{1}^{2}-18 v a_{1} \\
& -3 a_{1}^{2}+6 a_{1}=0, \\
& \psi^{3}(\xi): 24 d v^{3} a_{1}+12 d v^{2} a_{1}^{2}-72 d v^{2} a_{1}-24 d v a_{1}^{2} \\
& -12 \lambda v^{2} a_{1}-6 \lambda v a_{1}^{2}+72 d v a_{1}+12 d a_{1}^{2}+24 \lambda v a_{1} \\
& +6 \lambda a_{1}^{2}-24 d a_{1}-12 \lambda a_{1}=0, \\
& \psi^{2}(\xi):-36 d^{2} v^{3} a_{1}-18 d^{2} v^{2} a_{1}^{2}+108 d^{2} v^{2} a_{1} \\
& +36 d^{2} v a_{1}^{2}+36 d \lambda v^{2} a_{1}+18 d \lambda v a_{1}^{2}-108 d^{2} v a_{1} \\
& -18 d^{2} a_{1}^{2}-72 d \lambda v a_{1}-18 d \lambda a_{1}^{2}-7 \lambda^{2} v a_{1}-3 \lambda^{2} a_{1}^{2} \\
& -8 \mu v^{2} a_{1}-6 \mu v a_{1}^{2}+6 v^{2} a_{-1} a_{1}+2 v v a_{1}+36 d^{2} a_{1} \\
& +36 d \lambda a_{1}+7 \lambda^{2} a_{1}+16 \mu v a_{1}+6 \mu a_{1}^{2}-12 v a_{-1} a_{1} \\
& -2 V a_{1}-8 \mu a_{1}+3 v a_{1}+6 a_{-1} a_{1}-3 a_{1}=0, \\
& \psi(\xi): 24 d^{3} v^{3} a_{1}+12 d^{3} v^{2} a_{1}^{2}-72 d^{3} v^{2} a_{1}-24 d^{3} v a_{1}^{2} \\
& -36 d^{2} \lambda v^{2} a_{1}-18 d^{2} \lambda v a_{1}^{2}+72 d^{3} v a_{1}+12 d^{3} a_{1}^{2} \\
& +72 d^{2} \lambda v a_{1}+18 d^{2} \lambda a_{1}^{2}+14 d \lambda^{2} v a_{1}+6 d \lambda^{2} a_{1}^{2} \\
& +16 d \mu v^{2} a_{1}+12 d \mu v a_{1}^{2}-24 d v^{2} a_{-1} a_{1}-4 V d v a_{1} \\
& -24 d^{3} a_{1}-36 d^{2} \lambda a_{1}-14 d \lambda^{2} a_{1}-32 d \mu v a_{1} \\
& -12 d \mu a_{1}^{2}+48 d v a_{-1} a_{1}-\lambda^{3} a_{1}-8 \lambda \mu v a_{1}-6 \lambda \mu a_{1}^{2} \\
& +12 \lambda v a_{-1} a_{1}+4 V d a_{1}+2 V \lambda a_{1}+16 d \mu a_{1}-6 d v a_{1} \\
& -24 d a_{-1} a_{1}+8 \lambda \mu a_{1}-12 \lambda a_{-1} a_{1}+6 d a_{1} \\
& +3 \lambda a_{1}=0, \\
& \psi^{0}(\xi):-6 d^{4} v^{3} a_{1}-3 d^{4} v^{2} a_{1}^{2}+18 d^{4} v^{2} a_{1}+6 d^{4} v a_{1}^{2} \\
& +12 d^{3} \lambda v^{2} a_{1}+6 d^{3} \lambda v a_{1}^{2}-18 d^{4} v a_{1}-3 d^{4} a_{1}^{2} \\
& -24 d^{3} \lambda v a_{1}-6 d^{3} \lambda a_{1}^{2}-7 d^{2} \lambda^{2} v a_{1}-3 d^{2} \lambda^{2} a_{1}^{2} \\
& -8 d^{2} \mu v^{2} a_{1}-6 d^{2} \mu v a_{1}^{2}+6 d^{2} v^{3} a_{-1}+36 d^{2} v^{2} a_{-1} a_{1} \\
& +2 V d^{2} v a_{1}+6 d^{4} a_{1}+12 d^{3} \lambda a_{1}+7 d^{2} \lambda^{2} a_{1} \\
& +16 d^{2} \mu v a_{1}+6 d^{2} \mu a_{1}^{2}-18 d^{2} v^{2} a_{-1}-72 d^{2} v a_{-1} a_{1} \\
& +d \lambda^{3} a_{1}+8 d \lambda \mu v a_{1}+6 d \lambda \mu a_{1}^{2}-6 d \lambda v^{2} a_{-1}
\end{aligned}
$$

$$
\begin{aligned}
& -36 d \lambda v a_{-1} a_{1}-2 V d^{2} a_{1}-2 V d \lambda a_{1}-8 d^{2} \mu a_{1} \\
& +18 d^{2} v a_{-1}+3 d^{2} v a_{1}+36 d^{2} a_{-1} a_{1}-8 d \lambda \mu a_{1} \\
& +12 d \lambda v a_{-1}+36 d \lambda a_{-1} a_{1}-\lambda^{2} \mu a_{1}+\lambda^{2} v a_{-1} \\
& +6 \lambda^{2} a_{-1} a_{1}-2 \mu^{2} v a_{1}-3 \mu^{2} a_{1}^{2}+2 \mu v^{2} a_{-1} \\
& +12 \mu v a_{-1} a_{1}-3 v^{2} a_{-1}^{2}+2 V \mu a_{1}-2 V v a_{-1} \\
& -6 d^{2} a_{-1}-3 d^{2} a_{1}-6 d \lambda a_{-1}-3 d \lambda a_{1}-\lambda^{2} a_{-1} \\
& +2 \mu^{2} a_{1}-4 \mu v a_{-1}-12 \mu a_{-1} a_{1}+6 v a_{-1}^{2}+2 V a_{-1} \\
& +2 \mu a_{-1}+3 \mu a_{1}-3 v a_{-1}-3 a_{-1}^{2}+3 a_{-1}=0, \\
& \psi^{-1}(\xi):-24 d^{3} v^{3} a_{-1}-24 d^{3} v^{2} a_{-1} a_{1}+72 d^{3} v^{2} a_{-1} \\
& +48 d^{3} v a_{-1} a_{1}+36 d^{2} \lambda v^{2} a_{-1}+36 d^{2} \lambda v a_{-1} a_{1} \\
& -72 d^{3} v a_{-1}-24 d^{3} a_{-1} a_{1}-72 d^{2} \lambda v a_{-1} \\
& -36 d^{2} \lambda a_{-1} a_{1}-14 d \lambda^{2} v a_{-1}-12 d \lambda^{2} a_{-1} a_{1} \\
& -16 d \mu v^{2} a_{-1}-24 d \mu v a_{-1} a_{1}+12 d v^{2} a_{-1}^{2} \\
& +4 V d v a_{-1}+24 d^{3} a_{-1}+36 d^{2} \lambda a_{-1}+14 d \lambda^{2} a_{-1} \\
& +32 d \mu v a_{-1}+24 d \mu a_{-1} a_{1}-24 d v a_{-1}^{2}+\lambda^{3} a_{-1} \\
& +8 \lambda \mu v a_{-1}+12 \lambda \mu a_{-1} a_{1}-6 \lambda v a_{-1}^{2}-4 V d a_{-1} \\
& -2 V \lambda a_{-1}-16 d \mu a_{-1}+6 d v a_{-1}+12 d a_{-1}^{2}-8 \lambda \mu a_{-1} \\
& +6 \lambda a_{-1}^{2}-6 d a_{-1}-3 \lambda a_{-1}=0, \\
& \psi^{-2}(\xi): 36 d^{4} v^{3} a_{-1}+6 d^{4} v^{2} a_{-1} a_{1}-108 d^{4} v^{2} a_{-1} \\
& -12 d^{4} v a_{-1} a_{1}-72 d^{3} \lambda v^{2} a_{-1}-12 d^{3} \lambda v a_{-1} a_{1} \\
& +108 d^{4} v a_{-1}+6 d^{4} a_{-1} a_{1}+144 d^{3} \lambda v a_{-1} \\
& +12 d^{3} \lambda a_{-1} a_{1}+43 d^{2} \lambda^{2} v a_{-1}+6 d^{2} \lambda^{2} a_{-1} a_{1} \\
& +44 d^{2} \mu v^{2} a_{-1}+12 d^{2} \mu v a_{-1} a_{1}-18 d^{2} v^{2} a_{-1}^{2} \\
& -2 V d^{2} v a_{-1}-36 d^{4} a_{-1}-72 d^{3} \lambda a_{-1}-43 d^{2} \lambda^{2} a_{-1} \\
& -88 d^{2} \mu v a_{-1}-12 d^{2} \mu a_{-1} a_{1}+36 d^{2} v a_{-1}^{2}-7 d \lambda^{3} a_{-1} \\
& -44 d \lambda \mu v a_{-1}-12 d \lambda \mu a_{-1} a_{1}+18 d \lambda v a_{-1}^{2} \\
& +2 V d^{2} a_{-1}+2 V d \lambda a_{-1}+44 d^{2} \mu a_{-1}-3 d^{2} v a_{-1} \\
& -18 d^{2} a_{-1}^{2}+44 d \lambda \mu a_{-1}-18 d \lambda a_{-1}^{2}+7 \lambda^{2} \mu a_{-1} \\
& -3 \lambda^{2} a_{-1}^{2}+8 \mu^{2} v a_{-1}+6 \mu^{2} a_{-1} a_{1}-6 \mu v a_{-1}^{2} \\
& -2 V \mu a_{-1}+3 d^{2} a_{-1}+3 d \lambda a_{-1}-8 \mu^{2} a_{-1}+6 \mu a_{-1}^{2} \\
& -3 \mu a_{-1}=0 \text {, }
\end{aligned}
$$




$$
\begin{aligned}
& \psi^{-3}(\xi):-24 d^{5} v^{3} a_{-1}+72 d^{5} v^{2} a_{-1}+60 d^{4} \lambda v^{2} a_{-1} \\
& -72 d^{5} v a_{-1}-120 d^{4} \lambda v a_{-1}-48 d^{3} \lambda^{2} v a_{-1} \\
& -48 d^{3} \mu v^{2} a_{-1}+12 d^{3} v^{2} a_{-1}^{2}+24 d^{5} a_{-1}+60 d^{4} \lambda a_{-1} \\
& +48 d^{3} \lambda^{2} a_{-1}+96 d^{3} \mu v a_{-1}-24 d^{3} v a_{-1}^{2} \\
& +12 d^{2} \lambda^{3} a_{-1}+72 d^{2} \lambda \mu v a_{-1}-18 d^{2} \lambda v a_{-1}^{2} \\
& -48 d^{3} \mu a_{-1}+12 d^{3} a_{-1}^{2}-72 d^{2} \lambda \mu a_{-1}+18 d^{2} \lambda a_{-1}^{2} \\
& -24 d \lambda^{2} \mu a_{-1}+6 d \lambda^{2} a_{-1}^{2}-24 d \mu^{2} v a_{-1}+12 d \mu v a_{-1}^{2} \\
& +24 d \mu^{2} a_{-1}-12 d \mu a_{-1}^{2}+12 \lambda \mu^{2} a_{-1}-6 \lambda \mu a_{-1}^{2}=0, \\
& \psi^{-4}(\xi): 6 d^{6} v^{3} a_{-1}-18 d^{6} v^{2} a_{-1}-18 d^{5} \lambda v^{2} a_{-1} \\
& +18 d^{6} v a_{-1}+36 d^{5} \lambda v a_{-1}+18 d^{4} \lambda^{2} v a_{-1} \\
& +18 d^{4} \mu v^{2} a_{-1}-3 d^{4} v^{2} a_{-1}^{2}-6 d^{6} a_{-1}-18 d^{5} \lambda a_{-1} \\
& -18 d^{4} \lambda^{2} a_{-1}-36 d^{4} \mu v a_{-1}+6 d^{4} v a_{-1}^{2}-6 d^{3} \lambda^{3} a_{-1} \\
& -36 d^{3} \lambda \mu v a_{-1}+6 d^{3} \lambda v a_{-1}^{2}+18 d^{4} \mu a_{-1}-3 d^{4} a_{-1}^{2} \\
& +36 d^{3} \lambda \mu a_{-1}-6 d^{3} \lambda a_{-1}^{2}+18 d^{2} \lambda^{2} \mu a_{-1} \\
& -3 d^{2} \lambda^{2} a_{-1}^{2}+18 d^{2} \mu^{2} v a_{-1}-6 d^{2} \mu v a_{-1}^{2} \\
& -18 d^{2} \mu^{2} a_{-1}+6 d^{2} \mu a_{-1}^{2}-18 d \lambda \mu^{2} a_{-1}+6 d \lambda \mu a_{-1}^{2} \\
& +6 \mu^{3} a_{-1}-3 \mu^{2} a_{-1}^{2}=0 \text {. }
\end{aligned}
$$

Using the symbolic computation software Maple 17 to solve the above system in (48), we obtain the following three cases of unknown constants $a_{0}, a_{1}, a_{-1}, d$, and $V$.

Case 1.

$$
\begin{gathered}
a_{1}=0, \\
a_{-1}=2 d^{2} v-2 d^{2}-2 d \lambda+2 \mu, \\
V=\frac{1}{2} \lambda^{2}-2 \mu \nu+2 \mu-\frac{3}{2},
\end{gathered}
$$

$a_{0}, \mu, \lambda, v, d$ are arbitrary constants.
Case 2.

$$
\begin{gathered}
a_{1}=-2 v+2, \\
a_{-1}=0, \\
V=\frac{1}{2} \lambda^{2}-2 \mu \nu+2 \mu-\frac{3}{2},
\end{gathered}
$$

$a_{0}, \mu, \lambda, v, d$ are arbitrary constants.

Case 3.

$$
\begin{aligned}
a_{1} & =-2 v+2, \\
a_{-1} & =-\frac{\lambda^{2}-4 \mu v+4 \mu}{2(v-1)}, \\
d & =\frac{\lambda}{2(v-1)}, \\
V & =2 \lambda^{2}-8 \mu v+8 \mu-\frac{3}{2},
\end{aligned}
$$

$a_{0}, \mu, \lambda, v \neq 1$ are arbitrary constants.

All of the following exact solutions $u(x, y, z, t)$ of (28) are constructed by using the solutions of the generalized Riccati equation (A.1) which are classified into four families as mentioned in the Appendix. Then they are verified by substituting back into (30) with the aid of the Maple 17 package program to check that all of the solutions satisfy (30). In order to obtain the exact solutions $u(x, y, z, t)$ of (28), we must use the change of variable

$$
\xi=\frac{x^{\eta}}{\Gamma(1+\eta)}+\frac{y^{\beta}}{\Gamma(1+\beta)}+\frac{z^{\gamma}}{\Gamma(1+\gamma)}-\frac{V t^{\alpha}}{\Gamma(1+\alpha)},
$$

with the values of $V$ obtained from each case of unknown constants. Comparing (A.1) to (27), we have that $p=\lambda$, $q=(v-1), r=\mu$. For convenience, we set

$$
\Delta=\lambda^{2}-4 \mu(v-1) .
$$

The Exact Solutions of (28) Obtained Using the Unknown Constants of Case 1. Substituting (49) into (47) together with using (52) and (53), we obtain the following.

Family 1 . When $\Delta>0$ and $\lambda(v-1) \neq 0($ or $\mu(v-1) \neq 0)$, the hyperbolic function solutions of (28) are as follows:

$$
\begin{aligned}
u_{1}^{1}(x, y, z, t) & =\frac{2 d^{2} v-2 d^{2}-2 d \lambda+2 \mu}{d-(1 /(2(v-1)))[\lambda+\sqrt{\Delta} \tanh ((1 / 2) \sqrt{\Delta} \xi)]}+a_{0}, \\
u_{2}^{1}(x, y, z, t) & =\frac{2 d^{2} v-2 d^{2}-2 d \lambda+2 \mu}{d-(1 /(2(v-1)))[\lambda+\sqrt{\Delta} \operatorname{coth}((1 / 2) \sqrt{\Delta} \xi)]}+a_{0}, \\
u_{3,4}^{1}(x, y, z, t) & =\frac{2 d^{2} v-2 d^{2}-2 d \lambda+2 \mu}{d-(1 /(2(v-1)))[\lambda+\sqrt{\Delta}(\tanh (\sqrt{\Delta} \xi) \pm i \operatorname{sech}(\sqrt{\Delta} \xi))]}+a_{0},
\end{aligned}
$$




$$
\begin{aligned}
& u_{5,6}^{1}(x, y, z, t)=\frac{2 d^{2} v-2 d^{2}-2 d \lambda+2 \mu}{d-(1 /(2(v-1)))[\lambda+\sqrt{\Delta}(\operatorname{coth}(\sqrt{\Delta} \xi) \pm \operatorname{csch}(\sqrt{\Delta} \xi))]}+a_{0}, \\
& u_{7}^{1}(x, y, z, t)=\frac{2 d^{2} v-2 d^{2}-2 d \lambda+2 \mu}{d-(1 /(4(v-1)))[2 \lambda+\sqrt{\Delta}(\tanh ((1 / 4) \sqrt{\Delta} \xi)+\operatorname{coth}((1 / 4) \sqrt{\Delta} \xi))]}+a_{0}, \\
& \left.u_{8,9}^{1}(x, y, z, t)=\frac{2 d^{2} v-2 d^{2}-2 d \lambda+2 \mu}{d+(1 /(2(v-1)))\left[-\lambda+\left( \pm \sqrt{\left(A^{2}+B^{2}\right) \Delta}-A \sqrt{\Delta} \cosh (\sqrt{\Delta} \xi)\right) /(A \sinh (\sqrt{\Delta} \xi)+B)\right.}\right]+a_{0}, \\
& u_{10,11}^{1}(x, y, z, t)=\frac{2 d^{2} v-2 d^{2}-2 d \lambda+2 \mu}{d+(1 /(2(v-1)))\left[-\lambda-\left( \pm \sqrt{\left(B^{2}-A^{2}\right) \Delta}+A \sqrt{\Delta} \sinh (\sqrt{\Delta} \xi)\right) /(A \cosh (\sqrt{\Delta} \xi)+B)\right]}+a_{0},
\end{aligned}
$$

where $A$ and $B$ are two nonzero real constants and satisfy $B^{2}-A^{2}>0$,

$$
\begin{aligned}
& u_{12}^{1}(x, y, z, t)=\frac{2 d^{2} v-2 d^{2}-2 d \lambda+2 \mu}{[d+2 \mu \cosh ((1 / 2) \sqrt{\Delta} \xi) /(\sqrt{\Delta} \sinh ((1 / 2) \sqrt{\Delta} \xi)-\lambda \cosh ((1 / 2) \sqrt{\Delta} \xi))]}+a_{0}, \\
& u_{13}^{1}(x, y, z, t)=\frac{2 d^{2} v-2 d^{2}-2 d \lambda+2 \mu}{[d-2 \mu \sinh ((1 / 2) \sqrt{\Delta} \xi) /(\lambda \sinh ((1 / 2) \sqrt{\Delta} \xi)-\sqrt{\Delta} \cosh ((1 / 2) \sqrt{\Delta} \xi))]}+a_{0}, \\
& u_{14,15}^{1}(x, y, z, t)=\frac{2 d^{2} v-2 d^{2}-2 d \lambda+2 \mu}{[d+2 \mu \cosh (\sqrt{\Delta} \xi) /(\sqrt{\Delta} \sinh (\sqrt{\Delta} \xi)-\lambda \cosh (\sqrt{\Delta} \xi) \pm i \sqrt{\Delta})]}+a_{0}, \\
& u_{16,17}^{1}(x, y, z, t)=\frac{2 d^{2} v-2 d^{2}-2 d \lambda+2 \mu}{[d+2 \mu \sinh (\sqrt{\Delta} \xi) /(-\lambda \sinh (\sqrt{\Delta} \xi)+\sqrt{\Delta} \cosh (\sqrt{\Delta} \xi) \pm \sqrt{\Delta})]}+a_{0}, \\
& u_{18}^{1}(x, y, z, t) \\
& =\frac{2 d^{2} v-2 d^{2}-2 d \lambda+2 \mu}{\left[d+4 \mu \sinh ((1 / 4) \sqrt{\Delta} \xi) \cosh ((1 / 4) \sqrt{\Delta} \xi) /\left(-2 \lambda \sinh ((1 / 4) \sqrt{\Delta} \xi) \cosh ((1 / 4) \sqrt{\Delta} \xi)+2 \sqrt{\Delta} \cosh ^{2}((1 / 4) \sqrt{\Delta} \xi)-\sqrt{\Delta}\right)\right]} \\
& \quad+a_{0} .
\end{aligned}
$$

Family 2. When $\Delta<0$ and $\lambda(v-1) \neq 0($ or $\mu(v-1) \neq 0)$, the trigonometric function solutions of (28) are as follows:

$$
\begin{aligned}
u_{19}^{1}(x, y, z, t) & =\frac{2 d^{2} v-2 d^{2}-2 d \lambda+2 \mu}{d+(1 /(2(v-1)))[-\lambda+\sqrt{-\Delta} \tan ((1 / 2) \sqrt{-\Delta} \xi)]}+a_{0}, \\
u_{20}^{1}(x, y, z, t) & =\frac{2 d^{2} v-2 d^{2}-2 d \lambda+2 \mu}{d-(1 /(2(v-1)))[\lambda+\sqrt{-\Delta} \cot ((1 / 2) \sqrt{-\Delta} \xi)]}+a_{0}, \\
u_{21,22}^{1}(x, y, z, t) & =\frac{2 d^{2} v-2 d^{2}-2 d \lambda+2 \mu}{d+(1 /(2(v-1)))[-\lambda+\sqrt{-\Delta}(\tan (\sqrt{-\Delta} \xi) \pm \sec (\sqrt{-\Delta} \xi))]}+a_{0}, \\
u_{23,24}^{1}(x, y, z, t) & =\frac{2 d^{2} v-2 d^{2}-2 d \lambda+2 \mu}{d-(1 /(2(v-1)))[\lambda+\sqrt{-\Delta}(\cot (\sqrt{-\Delta} \xi) \pm \csc (\sqrt{-\Delta} \xi))]}+a_{0},
\end{aligned}
$$




$$
\begin{aligned}
u_{25}^{1}(x, y, z, t) & =\frac{2 d^{2} v-2 d^{2}-2 d \lambda+2 \mu}{d+(1 /(4(v-1)))[-2 \lambda+\sqrt{-\Delta}(\tan ((1 / 4) \sqrt{-\Delta} \xi)-\cot ((1 / 4) \sqrt{-\Delta} \xi))]}+a_{0}, \\
u_{26,27}^{1}(x, y, z, t) & =\frac{2 d^{2} v-2 d^{2}-2 d \lambda+2 \mu}{d+(1 /(2(v-1)))\left[-\lambda+\left( \pm \sqrt{-\left(A^{2}-B^{2}\right) \Delta}-A \sqrt{-\Delta} \cos (\sqrt{-\Delta} \xi)\right) /(A \sin (\sqrt{-\Delta} \xi)+B)\right]}+a_{0}, \\
u_{28,29}^{1}(x, y, z, t) & =\frac{2 d^{2} v-2 d^{2}-2 d \lambda+2 \mu}{d+(1 /(2(v-1)))\left[-\lambda-\left( \pm \sqrt{-\left(A^{2}-B^{2}\right) \Delta}+A \sqrt{-\Delta} \cos (\sqrt{-\Delta} \xi)\right) /(A \sin (\sqrt{-\Delta} \xi)+B)\right]}+a_{0},
\end{aligned}
$$

where $A$ and $B$ are two nonzero real constants and satisfy $A^{2}-B^{2}>0$,

$$
\begin{aligned}
& u_{30}^{1}(x, y, z, t)=\frac{2 d^{2} v-2 d^{2}-2 d \lambda+2 \mu}{[d-2 \mu \cos ((1 / 2) \sqrt{-\Delta} \xi) /(\sqrt{-\Delta} \sin ((1 / 2) \sqrt{-\Delta} \xi)+\lambda \cos ((1 / 2) \sqrt{-\Delta} \xi))]}+a_{0}, \\
& u_{31}^{1}(x, y, z, t)=\frac{2 d^{2} v-2 d^{2}-2 d \lambda+2 \mu}{[d+2 \mu \sin ((1 / 2) \sqrt{-\Delta} \xi) /(-\lambda \sin ((1 / 2) \sqrt{-\Delta} \xi)+\sqrt{-\Delta} \cos ((1 / 2) \sqrt{-\Delta} \xi))]}+a_{0}, \\
& u_{32,33}^{1}(x, y, z, t)=\frac{2 d^{2} v-2 d^{2}-2 d \lambda+2 \mu}{[d-2 \mu \cos (\sqrt{-\Delta} \xi) /(\sqrt{-\Delta} \sin (\sqrt{-\Delta} \xi)+\lambda \cos (\sqrt{-\Delta} \xi) \pm \sqrt{-\Delta})]}+a_{0}, \\
& u_{34,35}^{1}(x, y, z, t)=\frac{2 d^{2} v-2 d^{2}-2 d \lambda+2 \mu}{[d+2 \mu \sin (\sqrt{-\Delta} \xi) /(-\lambda \sin (\sqrt{-\Delta} \xi)+\sqrt{-\Delta} \cos (\sqrt{-\Delta} \xi) \pm \sqrt{-\Delta})]}+a_{0}, \\
& u_{36}^{1}(x, y, z, t) \\
& =\frac{2 d^{2} v-2 d^{2}-2 d \lambda+2 \mu}{\left[d+4 \mu \sin ((1 / 4) \sqrt{-\Delta} \xi) \cos ((1 / 4) \sqrt{-\Delta} \xi) /\left(-2 \lambda \sin ((1 / 4) \sqrt{-\Delta} \xi) \cos ((1 / 4) \sqrt{-\Delta} \xi)+2 \sqrt{-\Delta} \cos ^{2}((1 / 4) \sqrt{-\Delta} \xi)-\sqrt{-\Delta}\right)\right]} \\
& \quad+a_{0} .
\end{aligned}
$$

Family 3. When $\mu=0$ and $\lambda(v-1) \neq 0$, the hyperbolic function solutions of (28) are as follows:

$$
\begin{aligned}
& u_{37}^{1}(x, y, z, t)=\frac{2 d^{2} v-2 d^{2}-2 d \lambda}{\left[d-\lambda c_{1} /\left((v-1) c_{1}+\cosh (\lambda \xi)-\sinh (\lambda \xi)\right)\right]}+a_{0}, \\
& u_{38}^{1}(x, y, z, t)=\frac{2 d^{2} v-2 d^{2}-2 d \lambda}{\left[d-\lambda(\cosh (\lambda \xi)+\sinh (\lambda \xi)) /\left((v-1)\left(c_{1}+\cosh (\lambda \xi)+\sinh (\lambda \xi)\right)\right)\right]}+a_{0},
\end{aligned}
$$

where $c_{1}$ is an arbitrary constant.

Family 4. When $\mu=\lambda=0$ and $\nu-1 \neq 0$, the rational function solution of (28) is as follows:

$$
u_{39}^{1}(x, y, z, t)=\frac{2 d^{2} v-2 d^{2}}{\left[d-1 /\left((v-1) \xi+c_{2}\right)\right]}+a_{0}
$$

where $c_{2}$ is an arbitrary constant.
The Exact Solutions of (28) Obtained Using the Unknown Constants of Case 2. Substituting (50) into (47) together with using (52) and (53), we obtain the following.

Family 1 . When $\Delta>0$ and $\lambda(v-1) \neq 0($ or $\mu(v-1) \neq 0)$, the hyperbolic function solutions of (28) are as follows:

$$
\begin{aligned}
& u_{1}^{2}(x, y, z, t)=a_{0}-2(v-1)\left[d-\frac{1}{2(v-1)}(\lambda\right. \\
& \left.\left.\quad+\sqrt{\Delta} \tanh \left(\frac{1}{2} \sqrt{\Delta} \xi\right)\right)\right],
\end{aligned}
$$




$$
\begin{aligned}
& u_{2}^{2}(x, y, z, t)=a_{0}-2(v-1)\left[d-\frac{1}{2(v-1)}(\lambda\right. \\
& \left.\left.+\sqrt{\Delta} \operatorname{coth}\left(\frac{1}{2} \sqrt{\Delta} \xi\right)\right)\right], \\
& u_{3,4}^{2}(x, y, z, t)=a_{0}-2(v-1)\left[d-\frac{1}{2(v-1)}(\lambda\right. \\
& \quad+\sqrt{\Delta}(\tanh (\sqrt{\Delta} \xi) \pm i \operatorname{sech}(\sqrt{\Delta} \xi)))] \\
& u_{5,6}^{2}(x, y, z, t)=a_{0}-2(v-1)\left[d-\frac{1}{2(v-1)}(\lambda\right. \\
& \quad+\sqrt{\Delta}(\operatorname{coth}(\sqrt{\Delta} \xi) \pm \operatorname{csch}(\sqrt{\Delta} \xi)))], \\
& u_{7}^{2}(x, y, z, t)=a_{0}-2(v-1)\left[d-\frac{1}{4(v-1)}(2 \lambda\right. \\
& \left.\left.\quad+\sqrt{\Delta}\left(\tanh \left(\frac{1}{4} \sqrt{\Delta} \xi\right)+\operatorname{coth}\left(\frac{1}{4} \sqrt{\Delta} \xi\right)\right)\right)\right]
\end{aligned}
$$

$$
\begin{gathered}
u_{8,9}^{2}(x, y, z, t)=a_{0}-2(v-1)\left[d+\frac{1}{2(v-1)}(-\lambda\right. \\
\left.\left.+\frac{ \pm \sqrt{\left(A^{2}+B^{2}\right) \Delta}-A \sqrt{\Delta} \cosh (\sqrt{\Delta} \xi)}{A \sinh (\sqrt{\Delta} \xi)+B}\right)\right],
\end{gathered}
$$

$$
u_{10,11}^{2}(x, y, z, t)=a_{0}-2(v-1)\left[d+\frac{1}{2(v-1)}(-\lambda\right.
$$$$
\left.\left.-\frac{ \pm \sqrt{\left(B^{2}-A^{2}\right) \Delta}+A \sqrt{\Delta} \sinh (\sqrt{\Delta} \xi)}{A \cosh (\sqrt{\Delta} \xi)+B}\right)\right],
$$

$$
\begin{aligned}
u_{12}^{2}(x, y, z, t) & =a_{0}-2(v-1)\left[d+\frac{2 \mu \cosh ((1 / 2) \sqrt{\Delta} \xi)}{\sqrt{\Delta} \sinh ((1 / 2) \sqrt{\Delta} \xi)-\lambda \cosh ((1 / 2) \sqrt{\Delta} \xi)}\right], \\
u_{13}^{2}(x, y, z, t) & =a_{0}-2(v-1)\left[d-\frac{2 \mu \sinh ((1 / 2) \sqrt{\Delta} \xi)}{\lambda \sinh ((1 / 2) \sqrt{\Delta} \xi)-\sqrt{\Delta} \cosh ((1 / 2) \sqrt{\Delta} \xi)}\right], \\
u_{14,15}^{2}(x, y, z, t) & =a_{0}-2(v-1)\left[d+\frac{2 \mu \cosh (\sqrt{\Delta} \xi)}{\sqrt{\Delta} \sinh (\sqrt{\Delta} \xi)-\lambda \cosh (\sqrt{\Delta} \xi) \pm i \sqrt{\Delta}}\right], \\
u_{16,17}^{2}(x, y, z, t) & =a_{0}-2(v-1)\left[d+\frac{2 \mu \sinh (\sqrt{\Delta} \xi)}{-\lambda \sinh (\sqrt{\Delta} \xi)+\sqrt{\Delta} \cosh (\sqrt{\Delta} \xi) \pm \sqrt{\Delta}}\right], \\
u_{18}^{2}(x, y, z, t) & =a_{0}-2(v-1)\left[d+\frac{4 \mu \sinh ((1 / 4) \sqrt{\Delta} \xi) \cosh ((1 / 4) \sqrt{\Delta} \xi)}{-2 \lambda \sinh ((1 / 4) \sqrt{\Delta} \xi) \cosh ((1 / 4) \sqrt{\Delta} \xi)+2 \sqrt{\Delta} \cosh ^{2}((1 / 4) \sqrt{\Delta} \xi)-\sqrt{\Delta}}\right] .
\end{aligned}
$$

Family 2. When $\Delta<0$ and $\lambda(v-1) \neq 0$ (or $\mu(v-$ $1) \neq 0$ ), the trigonometric function solutions of (28) are as follows:

$$
\begin{aligned}
& u_{19}^{2}(x, y, z, t)=a_{0}-2(v-1)\left[d+\frac{1}{2(v-1)}(-\lambda\right. \\
& \left.\left.+\sqrt{-\Delta} \tan \left(\frac{1}{2} \sqrt{-\Delta} \xi\right)\right)\right], \\
& u_{20}^{2}(x, y, z, t)=a_{0}-2(v-1)\left[d-\frac{1}{2(v-1)}(\lambda\right. \\
& \left.\left.+\sqrt{-\Delta} \cot \left(\frac{1}{2} \sqrt{-\Delta} \xi\right)\right)\right]
\end{aligned}
$$

$$
\begin{aligned}
& u_{21,22}^{2}(x, y, z, t)=a_{0}-2(v-1)\left[d+\frac{1}{2(v-1)}(-\lambda\right. \\
& +\sqrt{-\Delta}(\tan (\sqrt{-\Delta} \xi) \pm \sec (\sqrt{-\Delta} \xi)))] \\
& u_{23,24}^{2}(\xi)=a_{0}-2(v-1)\left[d-\frac{1}{2(v-1)}(\lambda\right. \\
& \quad+\sqrt{-\Delta}(\cot (\sqrt{-\Delta} \xi) \pm \csc (\sqrt{-\Delta} \xi)))] \\
& u_{25}^{2}(x, y, z, t)=a_{0}-2(v-1)\left[d+\frac{1}{4(v-1)}(-2 \lambda\right. \\
& \left.\left.\quad+\sqrt{-\Delta}\left(\tan \left(\frac{1}{4} \sqrt{-\Delta} \xi\right)-\cot \left(\frac{1}{4} \sqrt{-\Delta} \xi\right)\right)\right)\right]
\end{aligned}
$$




$$
\begin{gathered}
u_{26,27}^{2}(x, y, z, t)=a_{0}-2(v-1)\left[d+\frac{1}{2(v-1)}(-\lambda\right. \\
\left.\left.+\frac{ \pm \sqrt{-\left(A^{2}-B^{2}\right) \Delta}-A \sqrt{-\Delta} \cos (\sqrt{-\Delta} \xi)}{A \sin (\sqrt{-\Delta} \xi)+B}\right)\right]
\end{gathered}
$$

$$
\begin{gathered}
u_{28,29}^{2}(x, y, z, t)=a_{0}-2(v-1)\left[d+\frac{1}{2(v-1)}(-\lambda\right. \\
\left.\left.-\frac{ \pm \sqrt{-\left(A^{2}-B^{2}\right) \Delta}+A \sqrt{-\Delta} \cos (\sqrt{-\Delta} \xi)}{A \sin (\sqrt{-\Delta} \xi)+B}\right)\right],
\end{gathered}
$$

where $A$ and $B$ are two nonzero real constants and satisfy $A^{2}-$ $B^{2}>0$,

$$
\begin{aligned}
u_{30}^{2}(x, y, z, t) & =a_{0}-2(v-1)\left[d-\frac{2 \mu \cos ((1 / 2) \sqrt{-\Delta} \xi)}{\sqrt{-\Delta} \sin ((1 / 2) \sqrt{-\Delta} \xi)+\lambda \cos ((1 / 2) \sqrt{-\Delta} \xi)}\right], \\
u_{31}^{2}(x, y, z, t) & =a_{0}-2(v-1)\left[d+\frac{2 \mu \sin ((1 / 2) \sqrt{-\Delta} \xi)}{-\lambda \sin ((1 / 2) \sqrt{-\Delta} \xi)+\sqrt{-\Delta} \cos ((1 / 2) \sqrt{-\Delta} \xi)}\right], \\
u_{32,33}^{2}(x, y, z, t) & =a_{0}-2(v-1)\left[d-\frac{2 \mu \cos (\sqrt{-\Delta} \xi)}{\sqrt{-\Delta} \sin (\sqrt{-\Delta} \xi)+\lambda \cos (\sqrt{-\Delta} \xi) \pm \sqrt{-\Delta}}\right], \\
u_{34,35}^{2}(x, y, z, t) & =a_{0}-2(v-1)\left[d+\frac{2 \mu \sin (\sqrt{-\Delta} \xi)}{-\lambda \sin (\sqrt{-\Delta} \xi)+\sqrt{-\Delta} \cos (\sqrt{-\Delta} \xi) \pm \sqrt{-\Delta}}\right], \\
u_{36}^{2}(x, y, z, t) & =a_{0}-2(v-1)\left[d+\frac{4 \mu \sin ((1 / 4) \sqrt{-\Delta} \xi) \cos ((1 / 4) \sqrt{-\Delta} \xi)}{-2 \lambda \sin ((1 / 4) \sqrt{-\Delta} \xi) \cos ((1 / 4) \sqrt{-\Delta} \xi)+2 \sqrt{-\Delta} \cos ^{2}((1 / 4) \sqrt{-\Delta} \xi)-\sqrt{-\Delta}}\right] .
\end{aligned}
$$

Family 3. When $\mu=0$ and $\lambda(v-1) \neq 0$, the hyperbolic function solutions of (28) are as follows:

$$
\begin{gathered}
u_{37}^{2}(x, y, z, t)=a_{0}-2(v-1) \\
\cdot\left[d-\frac{\lambda c_{1}}{(v-1) c_{1}+\cosh (\lambda \xi)-\sinh (\lambda \xi)}\right], \\
u_{38}^{2}(x, y, z, t)=a_{0}-2(v-1) \\
\cdot\left[d-\frac{\lambda(\cosh (\lambda \xi)+\sinh (\lambda \xi))}{(v-1)\left(c_{1}+\cosh (\lambda \xi)+\sinh (\lambda \xi)\right)}\right],
\end{gathered}
$$

where $c_{1}$ is an arbitrary constant.
Family 4. When $\mu=\lambda=0$ and $\nu-1 \neq 0$, the rational function solution of (28) is as follows:

$$
u_{39}^{2}(x, y, z, t)=a_{0}-2(v-1)\left[d-\frac{1}{(v-1) \xi+c_{2}}\right],
$$

where $c_{2}$ is an arbitrary constant.

The Exact Solutions of (28) Obtained Using the Unknown Constants of Case 3. Substituting (51) into (47) together with using (52) and (53), we obtain the following.

Family 1 . When $\Delta>0$ and $\lambda(v-1) \neq 0($ or $\mu(v-1) \neq 0)$, the hyperbolic function solutions of (28) are as follows:

$$
\begin{aligned}
& u_{1}^{3}(x, y, z, t)=\frac{\Delta}{\sqrt{\Delta} \tanh ((1 / 2) \sqrt{\Delta} \xi)}+a_{0}+\sqrt{\Delta} \tanh \left(\frac{1}{2} \sqrt{\Delta} \xi\right), \\
& u_{2}^{3}(x, y, z, t)=\frac{\Delta}{\sqrt{\Delta} \operatorname{coth}((1 / 2) \sqrt{\Delta} \xi)}+a_{0}+\sqrt{\Delta} \operatorname{coth}\left(\frac{1}{2} \sqrt{\Delta} \xi\right), \\
& u_{3,4}^{3}(x, y, z, t)=\frac{\Delta}{\sqrt{\Delta}[\tanh (\sqrt{\Delta} \xi) \pm i \operatorname{sech}(\sqrt{\Delta} \xi)]}+a_{0}+\sqrt{\Delta}[\tanh (\sqrt{\Delta} \xi) \pm i \operatorname{sech}(\sqrt{\Delta} \xi)],
\end{aligned}
$$




$$
\begin{aligned}
u_{5,6}^{3}(x, y, z, t)= & \frac{\Delta}{\sqrt{\Delta}[\operatorname{coth}(\sqrt{\Delta} \xi) \pm \operatorname{csch}(\sqrt{\Delta} \xi)]}+a_{0}+\sqrt{\Delta}[\operatorname{coth}(\sqrt{\Delta} \xi) \pm i \operatorname{csch}(\sqrt{\Delta} \xi)] \\
u_{7}^{3}(x, y, z, t)= & \frac{\Delta}{(\sqrt{\Delta} / 2)[\tanh ((1 / 4) \sqrt{\Delta} \xi)+\operatorname{coth}((1 / 4) \sqrt{\Delta} \xi)]}+a_{0}+\frac{\sqrt{\Delta}}{2}\left[\tanh \left(\frac{1}{4} \sqrt{\Delta} \xi\right)+\operatorname{coth}\left(\frac{1}{4} \sqrt{\Delta} \xi\right)\right] \\
u_{8,9}^{3}(x, y, z, t)= & -\frac{\Delta}{\left[\left( \pm \sqrt{\left(A^{2}+B^{2}\right) \Delta}-A \sqrt{\Delta} \cosh (\sqrt{\Delta} \xi)\right) /(A \sinh (\sqrt{\Delta} \xi)+B)\right]}+a_{0} \\
& -\left[\frac{ \pm \sqrt{\left(A^{2}+B^{2}\right) \Delta}-A \sqrt{\Delta} \cosh (\sqrt{\Delta} \xi)}{A \sinh (\sqrt{\Delta} \xi)+B}\right] \\
u_{10,11}^{3}(x, y, z, t)= & \frac{\Delta}{\left[\left( \pm \sqrt{\left(-A^{2}+B^{2}\right) \Delta}+A \sqrt{\Delta} \sinh (\sqrt{\Delta} \xi)\right) /(A \cosh (\sqrt{\Delta} \xi)+B)\right]}+a_{0} \\
& +\left[\frac{ \pm \sqrt{\left(-A^{2}+B^{2}\right) \Delta}+A \sqrt{\Delta} \sinh (\sqrt{\Delta} \xi)}{A \cosh (\sqrt{\Delta} \xi)+B}\right]
\end{aligned}
$$

where $A$ and $B$ are two nonzero real constants and satisfy $B^{2}-A^{2}>0$.

$$
\begin{aligned}
& u_{12}^{3}(x, y, z, t)=-\frac{1}{2(v-1)} \frac{\Delta}{[\lambda /(2(v-1))+2 \mu \cosh ((1 / 2) \sqrt{\Delta} \xi) /(\sqrt{\Delta} \sinh ((1 / 2) \sqrt{\Delta} \xi)-\lambda \cosh ((1 / 2) \sqrt{\Delta} \xi))]}+a_{0}-2(v-1) \\
& \cdot\left[\frac{\lambda}{2(v-1)}+\frac{2 \mu \cosh ((1 / 2) \sqrt{\Delta} \xi)}{\sqrt{\Delta} \sinh ((1 / 2) \sqrt{\Delta} \xi)-\lambda \cosh ((1 / 2) \sqrt{\Delta} \xi)}\right] \text {, } \\
& u_{13}^{3}(x, y, z, t)=-\frac{1}{2(v-1)} \frac{\Delta}{[\lambda /(2(v-1))-2 \mu \sinh ((1 / 2) \sqrt{\Delta} \xi) /(\lambda \sinh ((1 / 2) \sqrt{\Delta} \xi)-\sqrt{\Delta} \cosh ((1 / 2) \sqrt{\Delta} \xi))]}+a_{0}-2(v-1) \\
& \cdot\left[\frac{\lambda}{2(v-1)}-\frac{2 \mu \sinh ((1 / 2) \sqrt{\Delta} \xi)}{\lambda \sinh ((1 / 2) \sqrt{\Delta} \xi)-\sqrt{\Delta} \cosh ((1 / 2) \sqrt{\Delta} \xi)}\right], \\
& u_{14,15}^{3}(x, y, z, t)=-\frac{1}{2(v-1)} \frac{\Delta}{[\lambda /(2(v-1))+2 \mu \cosh (\sqrt{\Delta} \xi) /(\sqrt{\Delta} \sinh (\sqrt{\Delta} \xi)-\lambda \cosh (\sqrt{\Delta} \xi) \pm i \sqrt{\Delta})]}+a_{0}-2(v-1) \\
& \cdot\left[\frac{\lambda}{2(v-1)}+\frac{2 \mu \cosh (\sqrt{\Delta} \xi)}{\sqrt{\Delta} \sinh (\sqrt{\Delta} \xi)-\lambda \cosh (\sqrt{\Delta} \xi) \pm i \sqrt{\Delta}}\right] \\
& u_{16,17}^{3}(x, y, z, t)=-\frac{1}{2(v-1)} \frac{\Delta}{[\lambda /(2(v-1))+2 \mu \sinh (\sqrt{\Delta} \xi) /(-\lambda \sinh (\sqrt{\Delta} \xi)+\sqrt{\Delta} \cosh (\sqrt{\Delta} \xi) \pm \sqrt{\Delta})]}+a_{0}-2(v-1) \\
& \cdot\left[\frac{\lambda}{2(v-1)}+\frac{2 \mu \sinh (\sqrt{\Delta} \xi)}{-\lambda \sinh (\sqrt{\Delta} \xi)+\sqrt{\Delta} \cosh (\sqrt{\Delta} \xi) \pm \sqrt{\Delta}}\right] \\
& u_{18}^{3}(x, y, z, t)=-\frac{1}{2(v-1)} \\
& \cdot \frac{\Delta}{\left[\lambda /(2(v-1))+4 \mu \sinh ((1 / 4) \sqrt{\Delta} \xi) \cosh ((1 / 4) \sqrt{\Delta} \xi) /\left(-2 \lambda \sinh ((1 / 4) \sqrt{\Delta} \xi) \cosh ((1 / 4) \sqrt{\Delta} \xi)+2 \sqrt{\Delta} \cosh ^{2}((1 / 4) \sqrt{\Delta} \xi)-\sqrt{\Delta}\right)\right]} \\
& +a_{0}-2(v-1)\left[\frac{\lambda}{2(v-1)}+\frac{4 \mu \sinh ((1 / 4) \sqrt{\Delta} \xi) \cosh ((1 / 4) \sqrt{\Delta} \xi)}{-2 \lambda \sinh ((1 / 4) \sqrt{\Delta} \xi) \cosh ((1 / 4) \sqrt{\Delta} \xi)+2 \sqrt{\Delta} \cosh ^{2}((1 / 4) \sqrt{\Delta} \xi)-\sqrt{\Delta}}\right] .
\end{aligned}
$$


Family 2. When $\Delta<0$ and $\lambda(v-1) \neq 0($ or $\mu(v-1) \neq 0)$, the trigonometric function solutions of (28) are as follows:

$$
\begin{aligned}
& u_{19}^{3}(x, y, z, t)=-\frac{\Delta}{\sqrt{-\Delta} \tan ((1 / 2) \sqrt{-\Delta} \xi)}+a_{0}-\sqrt{-\Delta} \tan \left(\frac{1}{2} \sqrt{-\Delta} \xi\right) \\
& u_{20}^{3}(x, y, z, t)=\frac{\Delta}{\sqrt{-\Delta} \cot ((1 / 2) \sqrt{-\Delta} \xi)}+a_{0}+\sqrt{-\Delta} \cot \left(\frac{1}{2} \sqrt{-\Delta} \xi\right) \\
& u_{21,22}^{3}(x, y, z, t)=-\frac{\Delta}{[\sqrt{-\Delta}(\tan (\sqrt{-\Delta} \xi) \pm \sec (\sqrt{-\Delta} \xi))]}+a_{0}-[\sqrt{-\Delta}(\tan (\sqrt{-\Delta} \xi) \pm \sec (\sqrt{-\Delta} \xi))] \\
& u_{23,24}^{3}(x, y, z, t)=\frac{\Delta}{[\sqrt{-\Delta}(\cot (\sqrt{-\Delta} \xi) \pm \csc (\sqrt{-\Delta} \xi))]}+a_{0}+[\sqrt{-\Delta}(\cot (\sqrt{-\Delta} \xi) \pm \csc (\sqrt{-\Delta} \xi))] \\
& u_{25}^{3}(x, y, z, t)=-\frac{\Delta}{(\sqrt{-\Delta} / 2)[\tan ((1 / 4) \sqrt{-\Delta} \xi)-\cot ((1 / 4) \sqrt{-\Delta} \xi)]}+a_{0} \\
& -\frac{\sqrt{-\Delta}}{2}\left[\tan \left(\frac{1}{4} \sqrt{-\Delta} \xi\right)-\cot \left(\frac{1}{4} \sqrt{-\Delta} \xi\right)\right] \\
& u_{26,27}^{3}(x, y, z, t)=-\frac{\Delta}{\left[\left( \pm \sqrt{-\left(A^{2}-B^{2}\right) \Delta}-A \sqrt{-\Delta} \cos (\sqrt{-\Delta} \xi)\right) / A \sin (\sqrt{-\Delta} \xi+B)\right]}+a_{0} \\
& -\left[\frac{ \pm \sqrt{-\left(A^{2}-B^{2}\right) \Delta}-A \sqrt{-\Delta} \cos (\sqrt{-\Delta} \xi)}{A \sin (\sqrt{-\Delta} \xi+B)}\right] \\
& u_{28,29}^{3}(x, y, z, t)=\frac{\Delta}{\left[\left( \pm \sqrt{-\left(A^{2}-B^{2}\right) \Delta}+A \sqrt{-\Delta} \cos (\sqrt{-\Delta} \xi)\right) /(A \sin (\sqrt{-\Delta} \xi)+B)\right]}+a_{0} \\
& +\left[\frac{ \pm \sqrt{-\left(A^{2}-B^{2}\right) \Delta}+A \sqrt{-\Delta} \cos (\sqrt{-\Delta} \xi)}{A \sin (\sqrt{-\Delta} \xi)+B}\right],
\end{aligned}
$$

where $A$ and $B$ are two nonzero real constants and satisfy $A^{2}-B^{2}>0$,

$$
\begin{aligned}
& u_{30}^{3}(x, y, z, t)=-\frac{1}{2(v-1)} \frac{\Delta}{[\lambda /(2(v-1))-2 \mu \cos ((1 / 2) \sqrt{-\Delta} \xi) /(\sqrt{-\Delta} \sin ((1 / 2) \sqrt{-\Delta} \xi)+\lambda \cos ((1 / 2) \sqrt{-\Delta} \xi))]}+a_{0}-2(v-1) \\
& \cdot\left[\frac{\lambda}{2(v-1)}-\frac{2 \mu \cos ((1 / 2) \sqrt{-\Delta} \xi)}{\sqrt{-\Delta} \sin ((1 / 2) \sqrt{-\Delta} \xi)+\lambda \cos ((1 / 2) \sqrt{-\Delta} \xi)}\right], \\
& u_{31}^{3}(x, y, z, t)=-\frac{1}{2(v-1)} \frac{\Delta}{[\lambda /(2(v-1))+2 \mu \sin ((1 / 2) \sqrt{-\Delta} \xi) /(-\lambda \sin ((1 / 2) \sqrt{-\Delta} \xi)+\sqrt{-\Delta} \cos ((1 / 2) \sqrt{-\Delta} \xi))]}+a_{0}-2(v-1) \\
& \cdot\left[\frac{\lambda}{2(v-1)}+\frac{2 \mu \sin ((1 / 2) \sqrt{-\Delta} \xi)}{-\lambda \sin ((1 / 2) \sqrt{-\Delta} \xi)+\sqrt{-\Delta} \cos ((1 / 2) \sqrt{-\Delta} \xi)}\right], \\
& u_{32,33}^{3}(x, y, z, t)=-\frac{1}{2(v-1)} \frac{\Delta}{\lambda /(2(v-1))-2 \mu \cos (\sqrt{-\Delta} \xi) /(\sqrt{-\Delta} \sin (\sqrt{-\Delta} \xi)+\lambda \cos (\sqrt{-\Delta} \xi) \pm \sqrt{-\Delta})]}+a_{0}-2(v-1) \\
& \quad \cdot\left[\frac{\lambda}{2(v-1)}-\frac{2 \mu \cos (\sqrt{-\Delta} \xi)}{\sqrt{-\Delta} \sin (\sqrt{-\Delta} \xi)+\lambda \cos (\sqrt{-\Delta} \xi) \pm \sqrt{-\Delta}}\right],
\end{aligned}
$$




$$
\begin{aligned}
& u_{34,35}^{3}(x, y, z, t)=-\frac{1}{2(v-1)} \frac{\Delta}{[\lambda /(2(v-1))+2 \mu \sin (\sqrt{-\Delta} \xi) /(-\lambda \sin (\sqrt{-\Delta} \xi)+\sqrt{-\Delta} \cos (\sqrt{-\Delta} \xi) \pm \sqrt{-\Delta})]}+a_{0}-2(v-1) \\
& \cdot\left[\frac{\lambda}{2(v-1)}+\frac{2 \mu \sin (\sqrt{-\Delta} \xi)}{-\lambda \sin (\sqrt{-\Delta} \xi)+\sqrt{-\Delta} \cos (\sqrt{-\Delta} \xi) \pm \sqrt{-\Delta}}\right], \\
& u_{36}^{3}(x, y, z, t)=-\frac{1}{2(v-1)} \\
& \cdot \frac{\Delta}{\left[\lambda /(2(v-1))+4 \mu \sin ((1 / 4) \sqrt{-\Delta} \xi) \cos ((1 / 4) \sqrt{-\Delta} \xi) /\left(-2 \lambda \sin ((1 / 4) \sqrt{-\Delta} \xi) \cos ((1 / 4) \sqrt{-\Delta} \xi)+2 \sqrt{-\Delta} \cos ^{2}((1 / 4) \sqrt{-\Delta} \xi)-\sqrt{-\Delta}\right)\right]} \\
& +a_{0}-2(v-1)\left[\frac{\lambda}{2(v-1)}+\frac{4 \mu \sin ((1 / 4) \sqrt{-\Delta} \xi) \cos ((1 / 4) \sqrt{-\Delta} \xi)}{-2 \lambda \sin ((1 / 4) \sqrt{-\Delta} \xi) \cos ((1 / 4) \sqrt{-\Delta} \xi)+2 \sqrt{-\Delta} \cos ^{2}((1 / 4) \sqrt{-\Delta} \xi)-\sqrt{-\Delta}}\right]
\end{aligned}
$$

Family 3. When $\mu=0$ and $\lambda(v-1) \neq 0$, the hyperbolic function solutions of (28) are as follows:

$$
\begin{aligned}
u_{37}^{3}(x, y, z, t)= & -\frac{1}{2(v-1)} \frac{\lambda^{2}}{\left[\lambda /(2(v-1))-\lambda c_{1} /\left((v-1) c_{1}+\cosh (\lambda \xi)-\sinh (\lambda \xi)\right)\right]}+a_{0} \\
& -2(v-1)\left[\frac{\lambda}{2(v-1)}-\frac{\lambda c_{1}}{\left.(v-1) c_{1}+\cosh (\lambda \xi)-\sinh (\lambda \xi)\right]},\right. \\
u_{38}^{3}(x, y, z, t)=- & \frac{\lambda^{2}}{\left[\lambda-2 \lambda(\cosh (\lambda \xi)+\sinh (\lambda \xi)) /\left(c_{1}+\cosh (\lambda \xi)+\sinh (\lambda \xi)\right)\right]}+a_{0} \\
& -\left[\lambda-\frac{2 \lambda(\cosh (\lambda \xi)+\sinh (\lambda \xi))}{c_{1}+\cosh (\lambda \xi)+\sinh (\lambda \xi)}\right],
\end{aligned}
$$

where $c_{1}$ is an arbitrary constant.

Family 4. When $\mu=\lambda=0$ and $\nu-1 \neq 0$, the rational function solution of (28) is as follows:

$$
u_{39}^{3}(x, y, z, t)=a_{0}-\frac{-2 v+2}{(v-1) \xi+c_{2}},
$$

where $c_{2}$ is an arbitrary constant.

Our solutions in (81)-(107) for Case 2 are more general than the exact solutions obtained in [68] in which the authors employed the generalized Riccati equation mapping method to seek the exact solutions of (28) with $\eta=\beta=\gamma=\alpha=$ 1. However, their work did not produce the exact solutions for Cases 1 and 3, that is, (54)-(80) and (108)-(134). In addition, our exact solutions in (54), (66), (105), and (107) are equivalent to that, which were constructed by the $\exp (-\Phi(\xi))$ method, in [66] in which they were expressed in (28), (29), (30), and (32), respectively. Furthermore, our exact solutions in (79) and (106) have the same mathematical structure as solution (29) in [67] using the exp-function method. Hence, the novel $\left(G^{\prime} / G\right)$-expansion method used in our work have provided more new forms of the exact solutions of (28) since the method gives more free parameters than the existing methods utilized previously.

\section{Some Graphical Representations of Some Solutions and Their Physical Explanations}

The exact explicit solutions of the $(3+1)$-dimensional nonlinear space-time fractional Jimbo-Miwa equation in (28) are very useful for describing the diverse types of solitary wave solutions in physics. In this section, we will therefore provide some graphical representations of some interesting exact solutions of the equation. In addition, we will also discuss the physical explanations of such exact solutions. The selected exact explicit solutions of (28), which are plotted only for $-15 \leq x, y \leq 15$ with various values of $\eta$, $\beta$, are characterized by their shapes and physical meanings. The used values of $\eta, \beta$ are mixed among $\eta=1,0.99,0.9$ and $\beta=1,0.99,0.8$. For the fixed values $\eta=0.9, \beta=0.8$, the absolute value of the selected exact solutions $|u(x, y, z, t)|$ is also plotted since the solutions $u(x, y, z, t)$ sometimes arise in a complex form or produce complex values according to the variations of the used parameters and the plotting domain. The selected exact solutions of (28) obtained using the $\left(G^{\prime} / G, 1 / G\right)$-expansion method and the novel $\left(G^{\prime} / G\right)$ expansion method are graphically portrayed as follows. The results in (36), (39), and (44), constructed using the $\left(G^{\prime} / G, 1 / G\right)$-expansion method, are chosen in presenting their graphs and physical meanings. By the novel $\left(G^{\prime} / G\right)$ expansion method, we show graphical representations and 


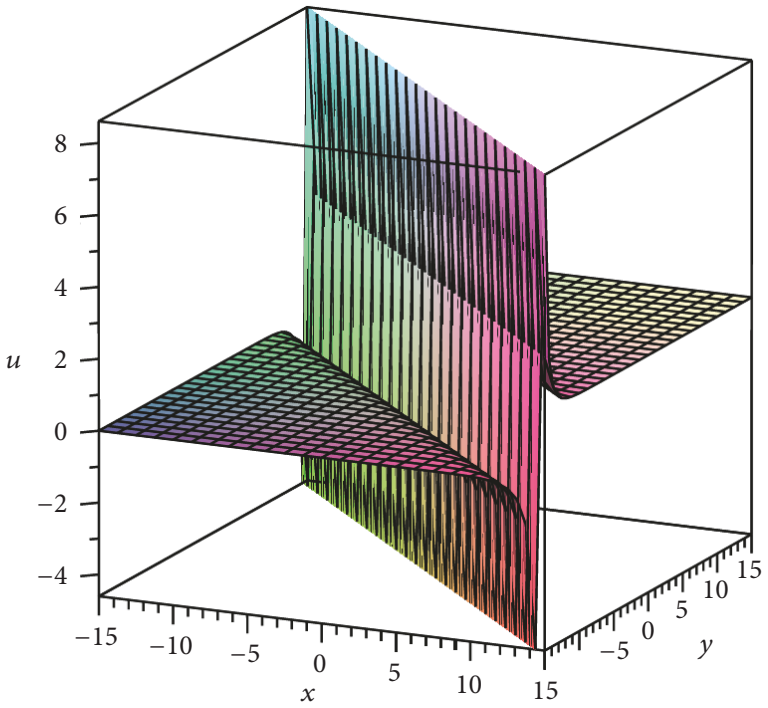

(a)

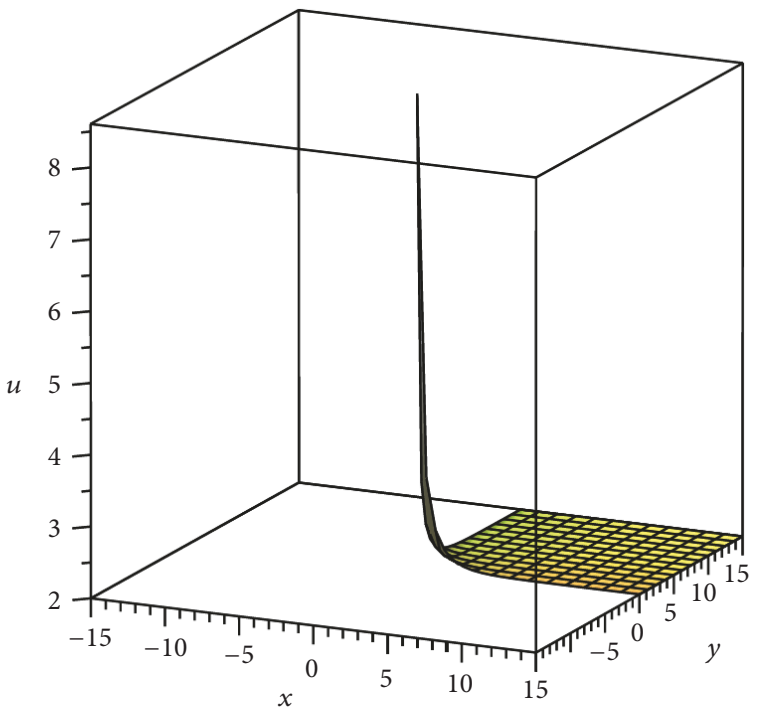

(c)

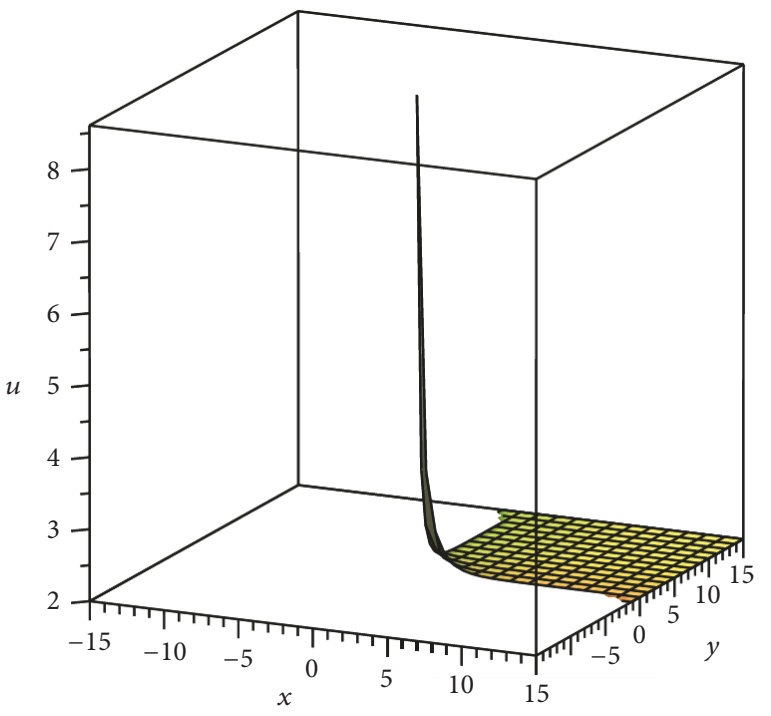

(b)

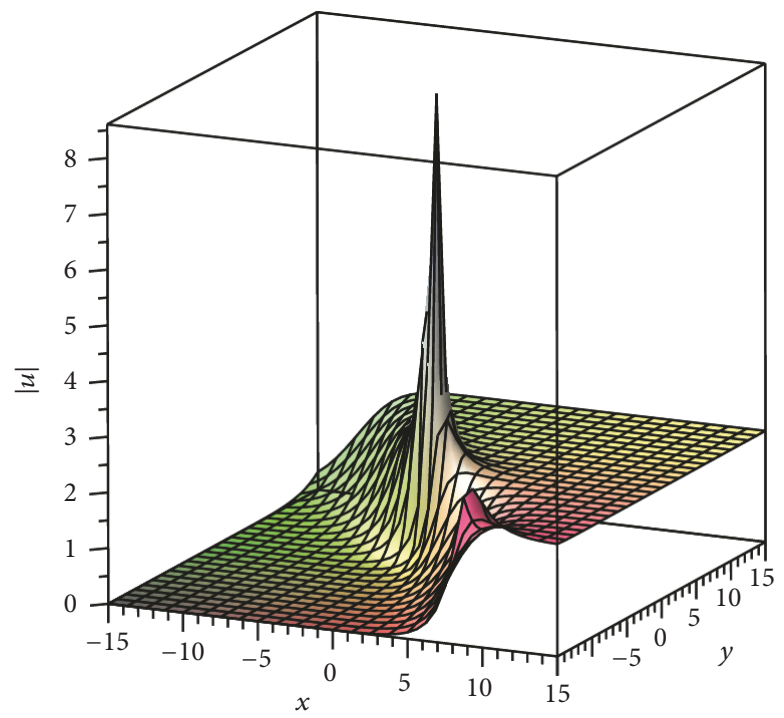

(d)

FIGURE 1: Associated plots of $u_{2}^{1}(x, y, z, t)$ in (36) with $-15 \leq x, y \leq 15$ using the $\left(G^{\prime} / G, 1 / G\right)$-expansion method: (a) solitary wave solution of singular kink type for $u_{2}^{1}(x, y, z, t)$ with $\eta=\beta=1$, (b) 1-soliton solitary wave solution for $u_{2}^{1}(x, y, z, t)$ with $\eta=\beta=0.99$, (c) 1-soliton solitary wave solution for $u_{2}^{1}(x, y, z, t)$ with $\eta=0.9, \beta=0.8$, and $(\mathrm{d})\left|u_{2}^{1}(x, y, z, t)\right|$ with $\eta=0.9, \beta=0.8$.

physical explanations of some chosen exact solutions of (28) for only Case 3.

Using the fixed values $\mu=0.5, \lambda=-1, A_{1}=2$, and $A_{2}=1$ and the various values of $\eta, \beta$, the hyperbolic function solutions of (28) generated employing $u_{2}^{1}(x, y, z, t)$ in (36) are demonstrated graphically as follows. The solution $u_{2}^{1}(x, y, z, t)$ with $\eta=\beta=1$, describing the solitary wave solution of singular kink type, is depicted in Figure 1(a). In addition, the exact 1-soliton solitary wave solutions $u_{2}^{1}(x, y, z, t)$ corresponding to $\eta=\beta=0.99$ and $\eta=0.9, \beta=$ 0.8 are shown in Figures $1(\mathrm{~b})$ and $1(\mathrm{c})$, respectively. Figure $1(\mathrm{~d})$ displays the graph of $\left|u_{2}^{1}(x, y, z, t)\right|$ with $\eta=0.9, \beta=0.8$.
For the fixed values $\mu=0, \lambda=1, A_{1}=2$, and $A_{1}=$ 1 and the specified variation of of $\eta, \beta$, the trigonometric exact solutions $u_{1}^{2}(x, y, z, t)$ in (39) of (28) are as follows. The solution $u_{1}^{2}(x, y, z, t)$ with $\eta=\beta=1$ describing the periodic traveling wave solution is depicted in Figure 2(a). The solution $u_{1}^{2}(x, y, z, t)$ with $\eta=\beta=0.99$ demonstrating singular double-soliton solution is presented in Figure 2(b). The graph, which is shown in Figure 2(c) and represents the singular multiple-soliton solution, corresponds to $u_{1}^{2}(x, y, z, t)$ with $\eta=0.9, \beta=0.8$. We can observe that their graphs are not plotted on the entire domain since these solutions have real values only on a certain part of the 


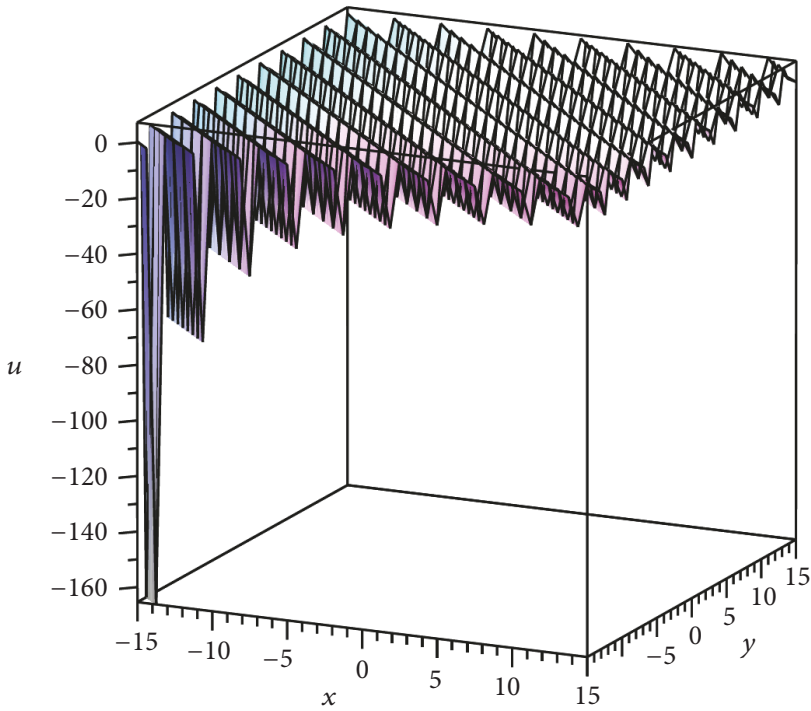

(a)

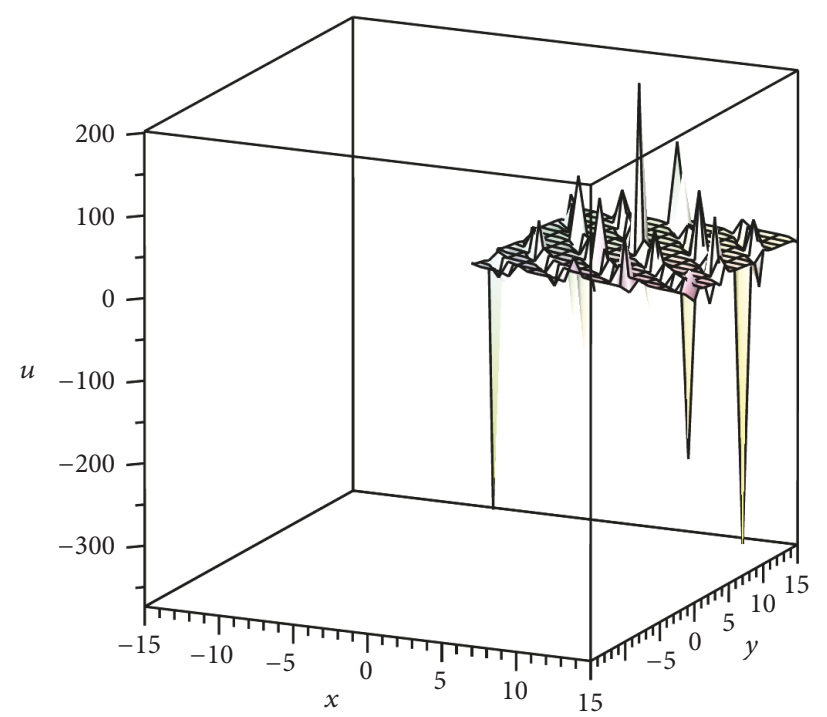

(c)

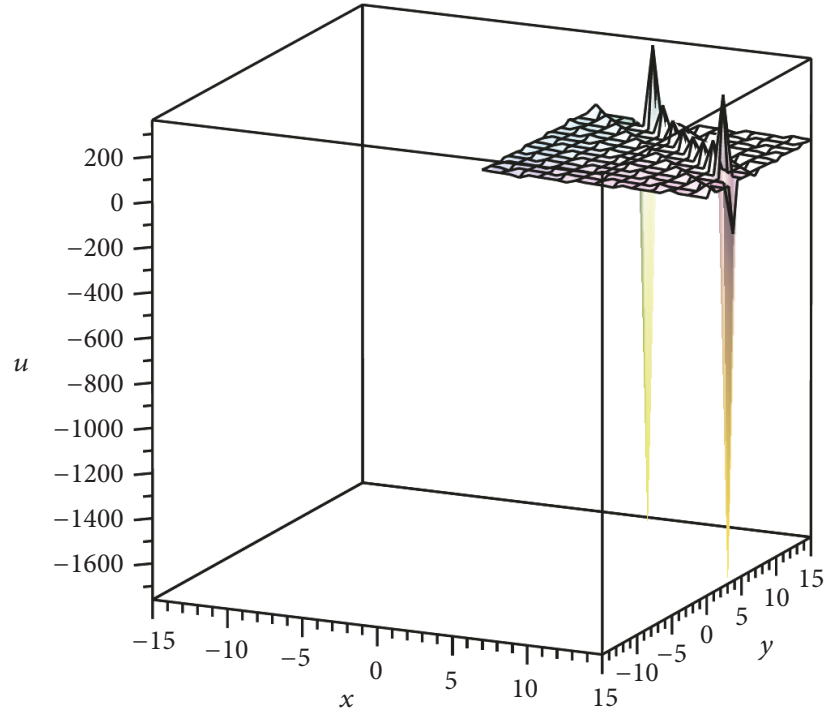

(b)

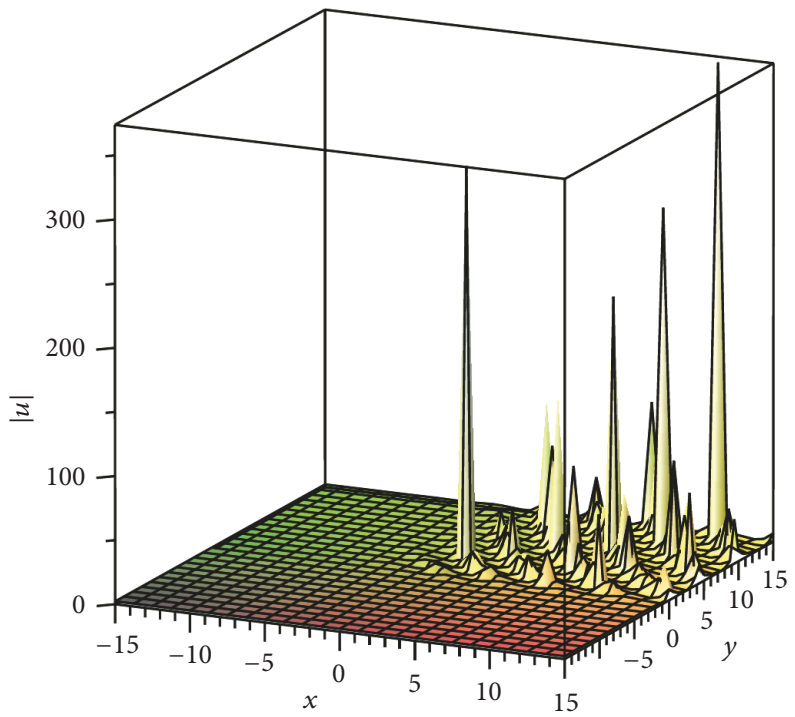

(d)

Figure 2: Associated plots of $u_{1}^{2}(x, y, z, t)$ in (39) with $-15 \leq x, y \leq 15$ using the $\left(G^{\prime} / G, 1 / G\right)$-expansion method: (a) periodic traveling wave solution for $u_{1}^{2}(x, y, z, t)$ with $\eta=\beta=1$, (b) singular double-soliton solution for $u_{1}^{2}(x, y, z, t)$ with $\eta=\beta=0.99$, (c) singular multiple-soliton solution for $u_{1}^{2}(x, y, z, t)$ with $\eta=0.9, \beta=0.8$, and (d) $\left|u_{1}^{2}(x, y, z, t)\right|$ with $\eta=0.9, \beta=0.8$.

domain. The graph of $\left|u_{1}^{2}(x, y, z, t)\right|$ with $\eta=0.9, \beta=0.8$ is shown in Figure 2(d).

Varying the values of $\eta, \beta$ as specified above, the rational function solution $u_{1}^{3}(x, y, z, t)$ in (44) of (28) provides the physical behaviors similar to the behaviors of $u_{2}^{1}(x, y, z, t)$ as shown previously. In other words, for the fixed values $\mu=0.5$, $\lambda=0, A_{1}=2$, and $A_{2}=1$, the solitary wave solution of singular kink type $u_{1}^{3}(x, y, z, t)$ with $\eta=\beta=1$ is plotted on the domain and represented in Figure 3(a). In addition, the solutions $u_{1}^{3}(x, y, z, t)$ with $\eta=\beta=0.99$ and $\eta=0.9$, $\beta=0.8$ characterize the same physical interpretations as demonstrated in Figures 3(b) and 3(c), respectively. They are 1-soliton solitary wave solutions occurring only for positive values of $x, y$ since they become complex for $x<0$ or $y<0$. The graph of $\left|u_{1}^{3}(x, y, z, t)\right|$ with $\eta=0.9, \beta=0.8$ is shown in Figure 3(d).

The following graphical representations are portrayed below using the chosen exact solutions constructed from the novel $\left(G^{\prime} / G\right)$-expansion method. For the fixed values $\mu=0.5$, $\lambda=1, v=0.5$, and $a_{0}=1$, the solutions generated using $u_{3}^{3}(x, y, z, t)$ in (110) of (28) with the specified values of $\eta, \beta$ are as follows. The solution $u_{3}^{3}(x, y, z, t)$ with $\eta=\beta=1$ gives the exact kink type solitary wave solution which occurs from one asymptotic state to another and approaches a constant at infinity. Its plot is shown in Figure 4(a). The discontinuous solitary wave solutions of kink type for $u_{3}^{3}(x, y, z, t)$ with $\eta=$ 


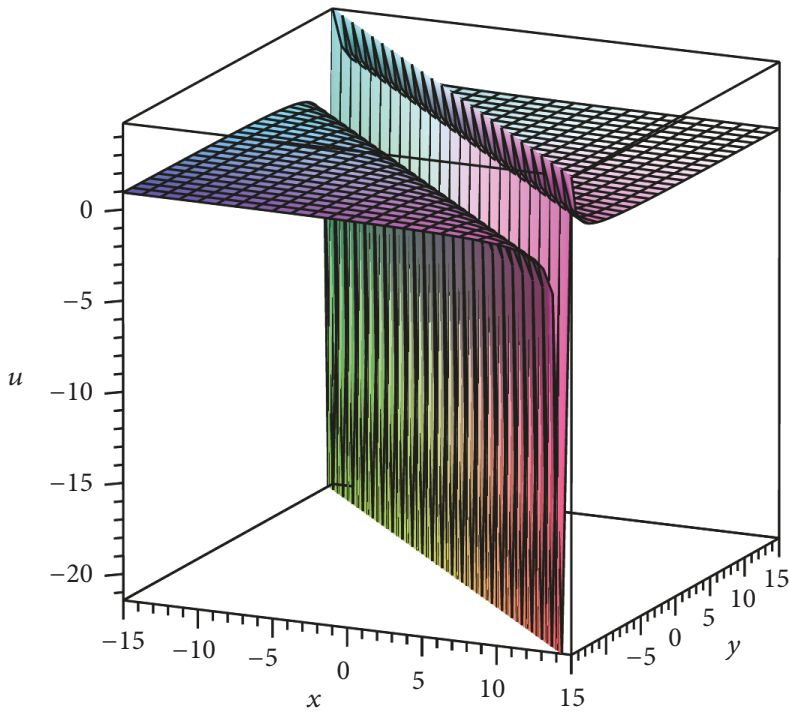

(a)

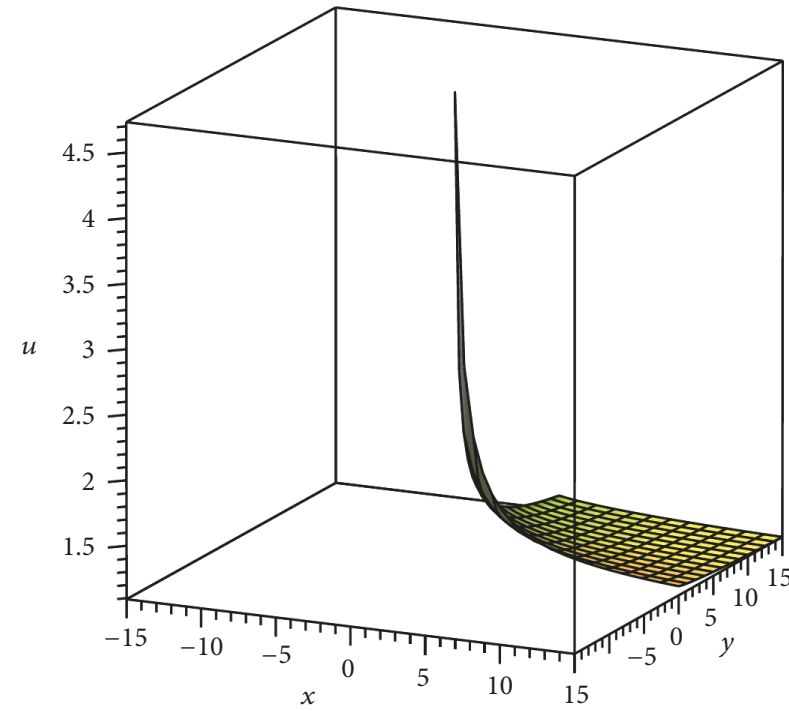

(c)

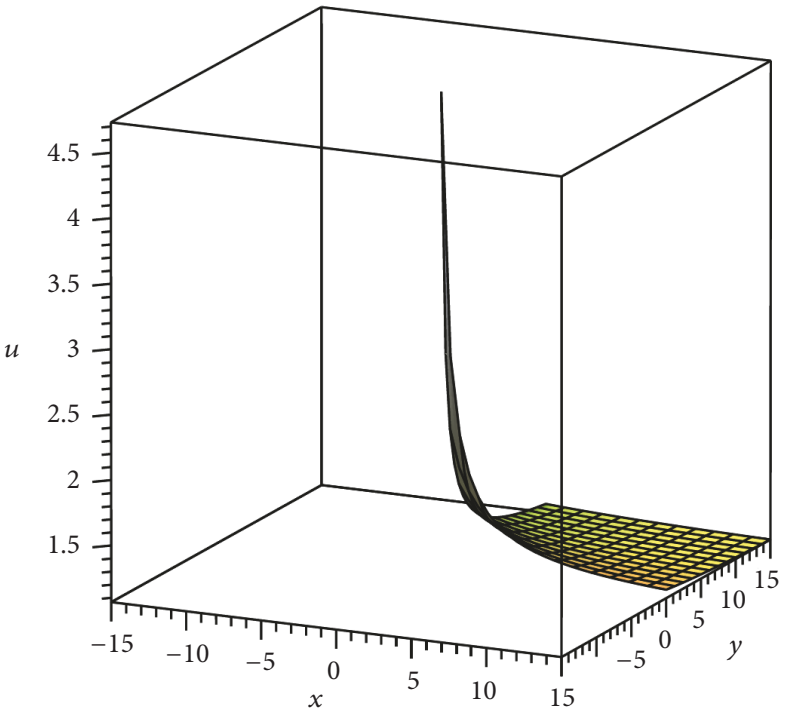

(b)

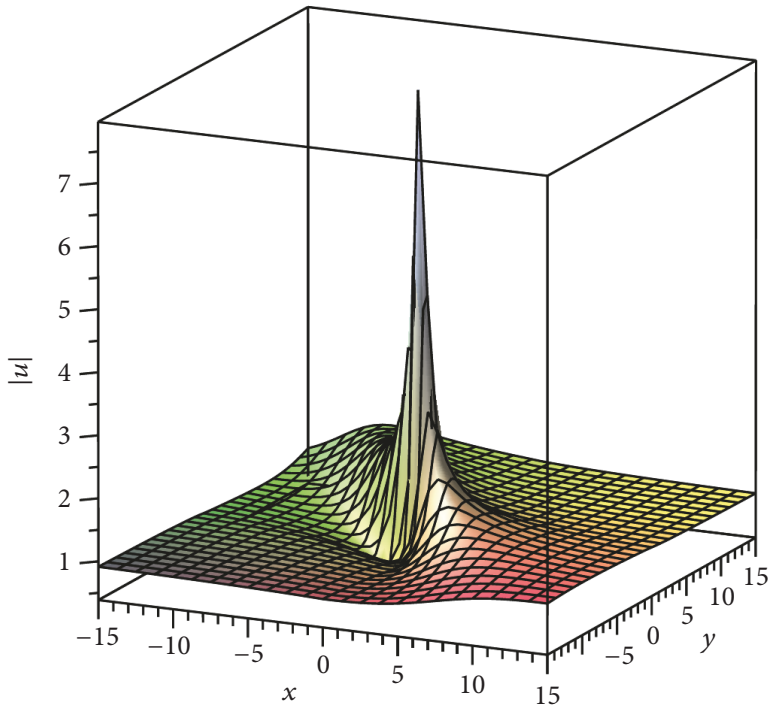

(d)

FIgURE 3: Associated plots of $u_{1}^{3}(x, y, z, t)$ in (44) with $-15 \leq x, y \leq 15$ using the $\left(G^{\prime} / G, 1 / G\right)$-expansion method: (a) solitary wave solution of singular kink type for $u_{1}^{3}(x, y, z, t)$ with $\eta=\beta=1$, (b) 1-soliton solitary wave solution for $u_{1}^{3}(x, y, z, t)$ with $\eta=\beta=0.99$, (c) 1-soliton solitary wave solution for $u_{1}^{3}(x, y, z, t)$ with $\eta=0.9, \beta=0.8$, and $(\mathrm{d})\left|u_{1}^{3}(x, y, z, t)\right|$ with $\eta=0.9, \beta=0.8$.

$\beta=0.99$ and $\eta=0.9, \beta=0.8$ are depicted in Figures $4(\mathrm{~b})$ and $4(c)$, respectively. Their graphs are not plotted on the entire domain since these solutions have complex values only on a certain part of the domain. The graph of $\left|u_{3}^{3}(x, y, z, t)\right|$ with $\eta=0.9, \beta=0.8$ is displayed in Figure 4(d).

The solution $u_{12}^{3}(x, y, z, t)$ of (28), expressed in (115), is characterized as the soliton solitary wave solution which is used to explain many physical phenomena such as soliton propagation in optical fibers. For the fixed values $\mu=0.5$, $\lambda=1, v=0.5$, and $a_{0}=1$ and on the specified domain, the exact soliton solitary wave solution $u_{12}^{3}(x, y, z, t)$, obtained using (115) with $\eta=\beta=1$, is represented in Figure 5(a). Figures 5(b) and 5(c) show the discontinuous shapes of the exact soliton solitary wave solutions $u_{12}^{3}(x, y, z, t)$ with $\eta=$ $\beta=0.99$ and $\eta=0.9, \beta=0.8$, respectively. The graph of $\left|u_{12}^{3}(x, y, z, t)\right|$ with $\eta=0.9, \beta=0.8$ is displayed in Figure 5(d).

The example of a singular periodic traveling wave solution of (28) can be investigated in the exact explicit solution $u_{25}^{3}(x, y, z, t)$ obtained from (124) with $\eta=\beta=1$ and the fixed values $\mu=1, \lambda=0.5, v=1.5$, and $a_{0}=1$. Its graph is represented in Figure 6(a). Using the same values of $\mu, \lambda, v$, and $a_{0}$, the exact singular double-soliton and multiple-soliton solutions for $u_{25}^{3}(x, y, z, t)$ corresponding to $\eta=\beta=0.99$ and $\eta=0.9, \beta=0.8$ are plotted in Figures 6(b) and 6(c), respectively. In these cases, we show the solutions only for 


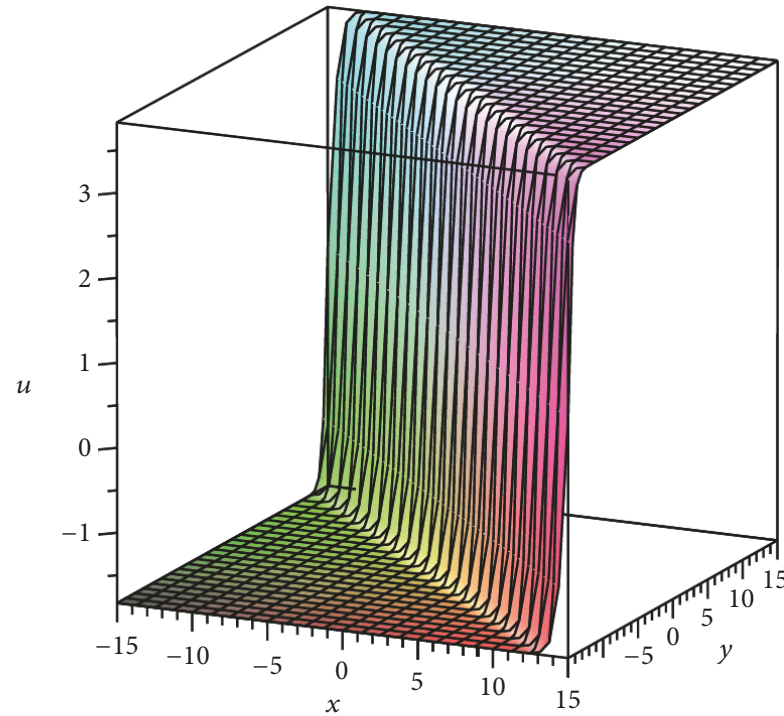

(a)

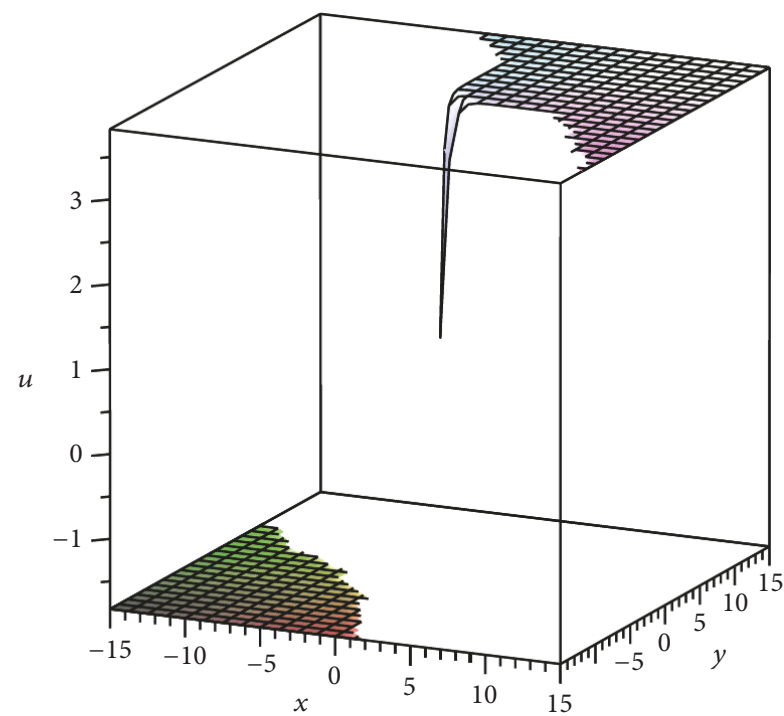

(c)

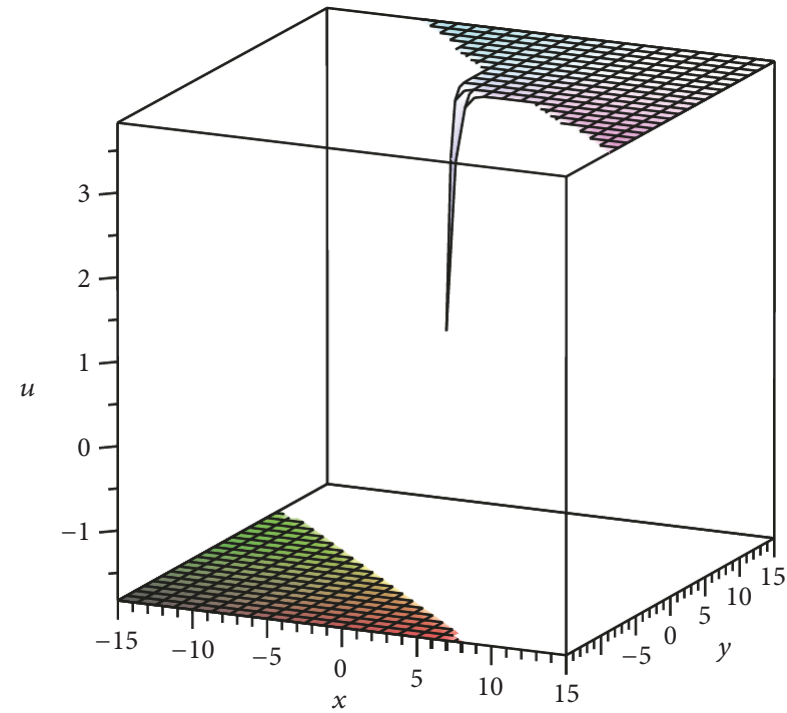

(b)

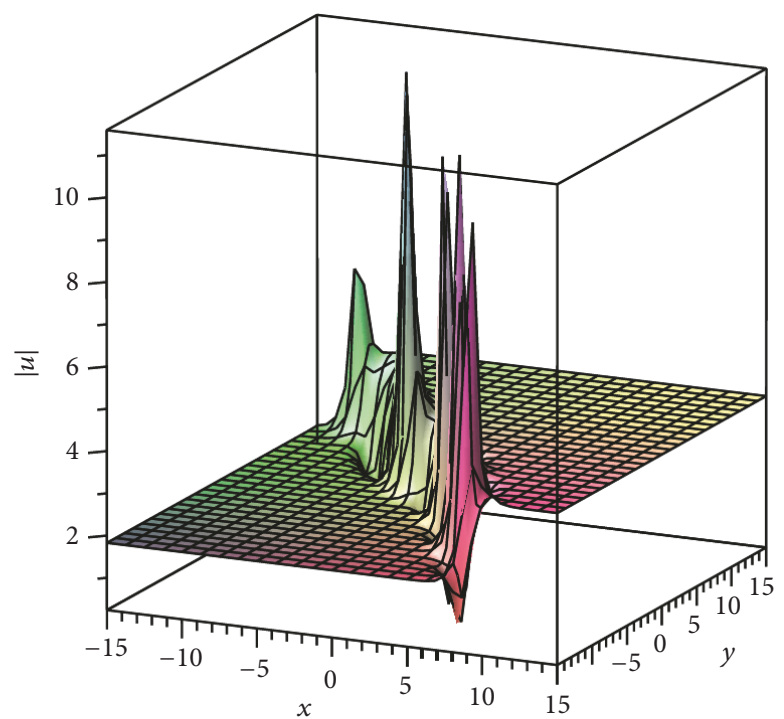

(d)

FIGURE 4: Associated plots of $u_{3}^{3}(x, y, z, t)$ in (110) with $-15 \leq x, y \leq 15$ using the novel $\left(G^{\prime} / G\right)$-expansion method: (a) solitary wave solution of kink type for $u_{3}^{3}(x, y, z, t)$ with $\eta=\beta=1$, (b) discontinuous solitary wave solution of kink type for $u_{3}^{3}(x, y, z, t)$ with $\eta=\beta=0.99$, (c) discontinuous solitary wave solution of kink type for $u_{3}^{3}(x, y, z, t)$ with $\eta=0.9, \beta=0.8$, and (d) $\left|u_{3}^{3}(x, y, z, t)\right|$ with $\eta=0.9, \beta=0.8$.

positive values of $x, y$ since they become complex for $x<0$ or $y<0$. Consequently, the graph of $\left|u_{25}^{3}(x, y, z, t)\right|$ with $\eta=0.9$, $\beta=0.8$ is plotted in Figure 6(d).

In Figure 7(a), we have presented the exact periodic traveling wave solution of $u_{30}^{3}(x, y, z, t)$ on the domain using $\eta=$ $\beta=1$ and the fixed values $\mu=1, \lambda=0.5, v=1.5, a_{0}=1, A=$ 1 , and $B=0.5$. Using the same values of $\mu, \lambda, v, a_{0}, A, B$, the exact singular double-soliton and multiple-soliton solutions for $u_{30}^{3}(x, y, z, t)$ corresponding to $\eta=\beta=0.99$ and $\eta=0.9$, $\beta=0.8$ are plotted in Figures $7(\mathrm{~b})$ and $7(\mathrm{c})$, respectively. In Figures $7(\mathrm{~b})$ and $7(\mathrm{c})$, the solutions $u_{30}^{3}(x, y, z, t)$ become complex values on the region in which the values of $x$ or $y$ are negative. In addition, the plot of $\left|u_{30}^{3}(x, y, z, t)\right|$ with $\eta=0.9$, $\beta=0.8$ is displayed in Figure $7(\mathrm{~d})$.

For the fixed values $\mu=0, \lambda=0.5, v=1.5, a_{0}=1$, and $c_{1}=1$, the exact explicit solution $u_{37}^{3}(x, y, z, t)$ of $(28)$, obtained using (132) and $\eta=\beta=1$, describes the solitary wave solution of singular kink type, which is shaped as in Figure 8(a). Figures 8(b) and 8(c) demonstrate the shapes of the exact 1-soliton solitary wave solutions of singular kink type using $u_{37}^{3}(x, y, z, t)$ with $\eta=\beta=0.99$ and $\eta=0.9$, $\beta=0.8$, respectively. The graph of $\left|u_{37}^{3}(x, y, z, t)\right|$ with $\eta=$ $0.9, \beta=0.8$ is displayed in Figure $8(\mathrm{~d})$.

For the fixed values $\mu=\lambda=0, v=1.5, a_{0}=1$, and $c_{1}=1$ and on the mentioned domain, the exact explicit 


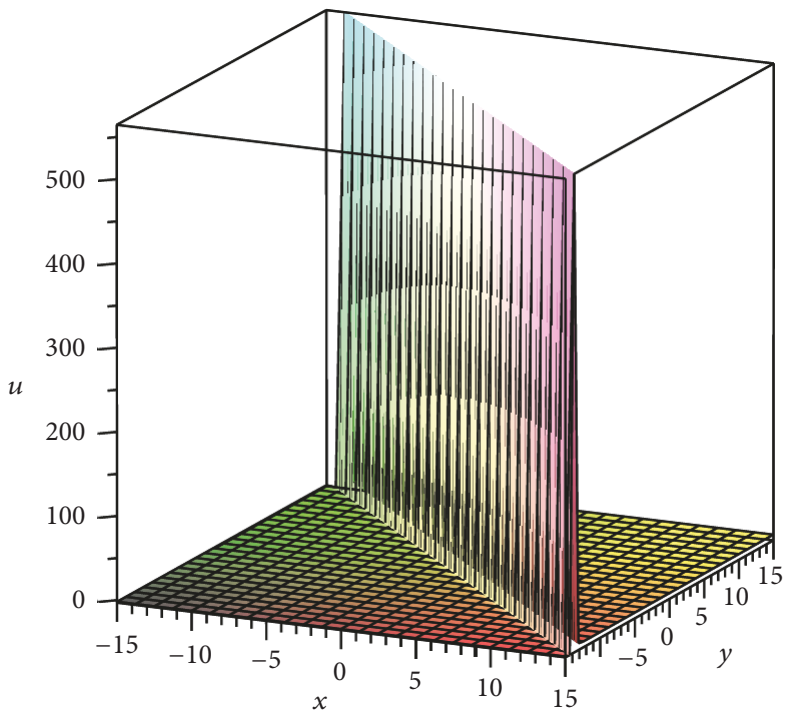

(a)

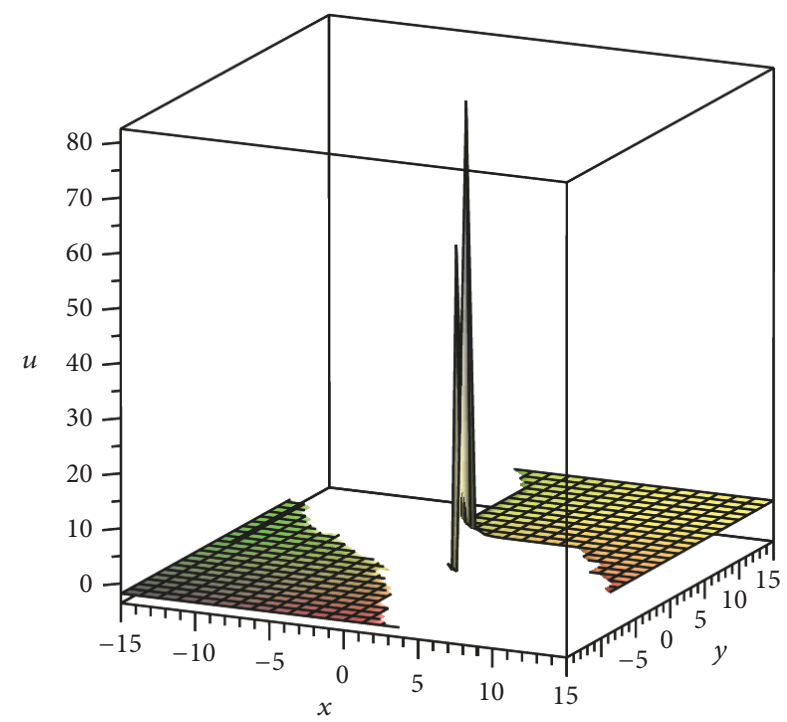

(c)

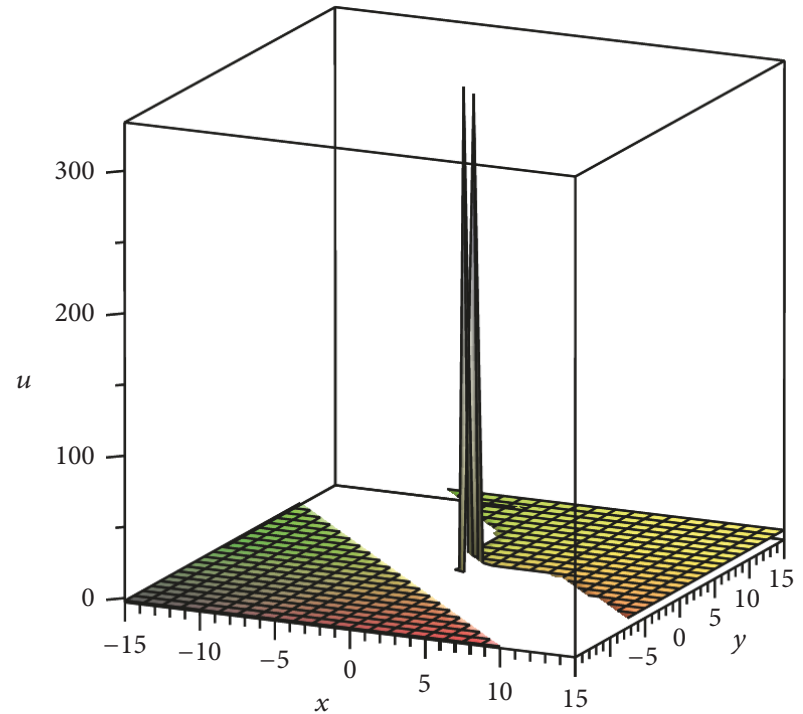

(b)

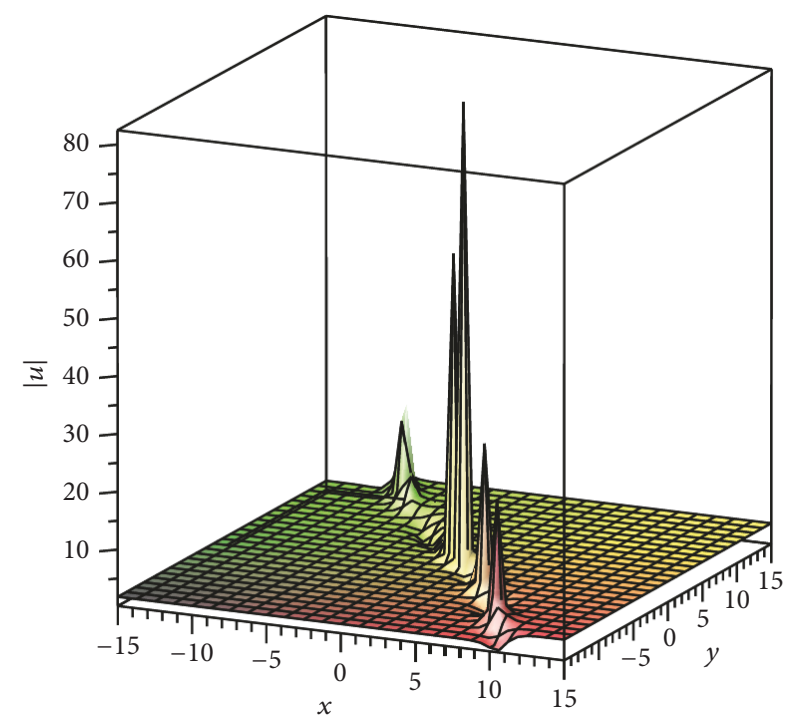

(d)

FIGURE 5: Associated plots of $u_{12}^{3}(x, y, z, t)$ in (115) with $-15 \leq x, y \leq 15$ using the novel $\left(G^{\prime} / G\right)$-expansion method: (a) Soliton solitary wave solution for $u_{12}^{3}(x, y, z, t)$ with $\eta=\beta=1$, (b) discontinuous soliton solitary wave solution for $u_{12}^{3}(x, y, z, t)$ with $\eta=\beta=0.99$, (c) discontinuous soliton solitary wave solution for $u_{12}^{3}(x, y, z, t)$ with $\eta=0.9, \beta=0.8$, and (d) $\left|u_{12}^{3}(x, y, z, t)\right|$ with $\eta=0.9, \beta=0.8$.

solutions $u_{39}^{3}(x, y, z, t)$ of (28) obtained using (134) and the various values of $\eta, \beta$ are shown in Figure 9. The solitary wave solution of singular kink type for $u_{39}^{3}(x, y, z, t)$ with $\eta=\beta=1$ has the shape as shown in Figure 9(a). Figures 9 (b) and 9(c) demonstrate the shapes of the exact 1-soliton solitary wave solutions $u_{39}^{3}(x, y, z, t)$ with $\eta=\beta=0.99$ and $\eta=0.9, \beta=0.8$, respectively. The graph of $\left|u_{39}^{3}(x, y, z, t)\right|$ with $\eta=0.9, \beta=0.8$ is displayed in Figure 9(d).

\section{Conclusions}

In this article, we have applied the $\left(G^{\prime} / G, 1 / G\right)$-expansion method and the novel $\left(G^{\prime} / G\right)$-expansion method to construct exact solitary wave solutions of the $(3+1)$-dimensional nonlinear space-time fractional Jimbo-Miwa equation expressed in (28) with the modified Riemann-Liouville derivative. With the aid of the fractional complex transform and the symbolic computation package Maple 17, abundant exact solutions, including generalized hyperbolic function solutions, generalized trigonometric function solutions, and rational function solutions, are obtained using the two methods. Furthermore, some of these exact solutions obtained by the methods have been graphically characterized into a variety of distinct physical structures such as a solitary wave solution of (singular) kink type, a 1-soliton solitary wave solution, a periodic traveling wave solution, a singular multiple-soliton 


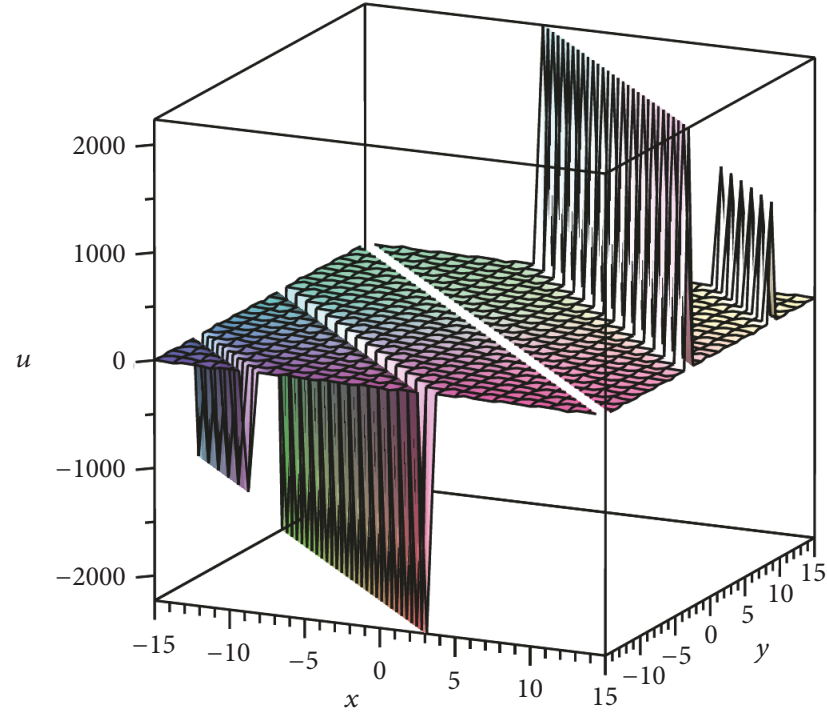

(a)

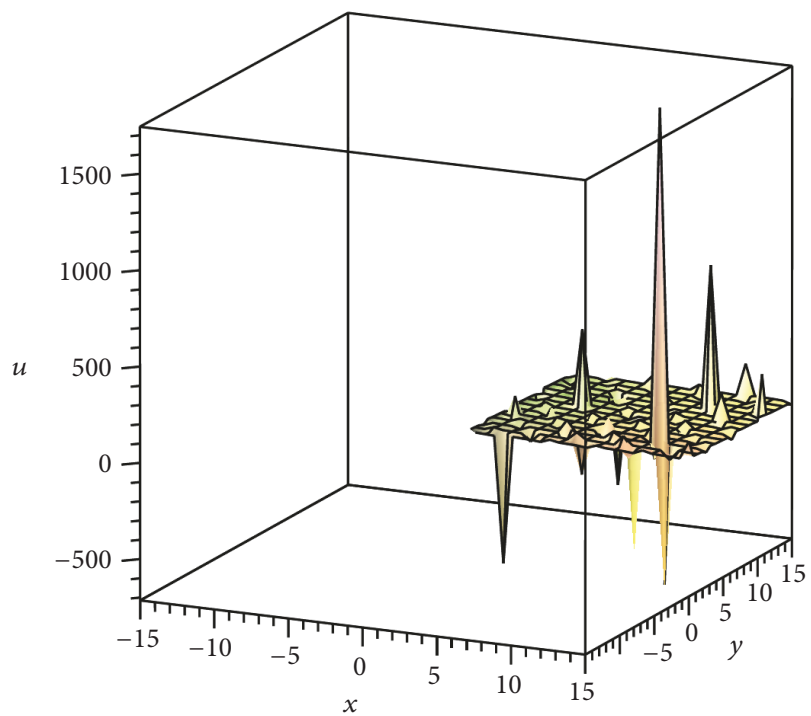

(c)

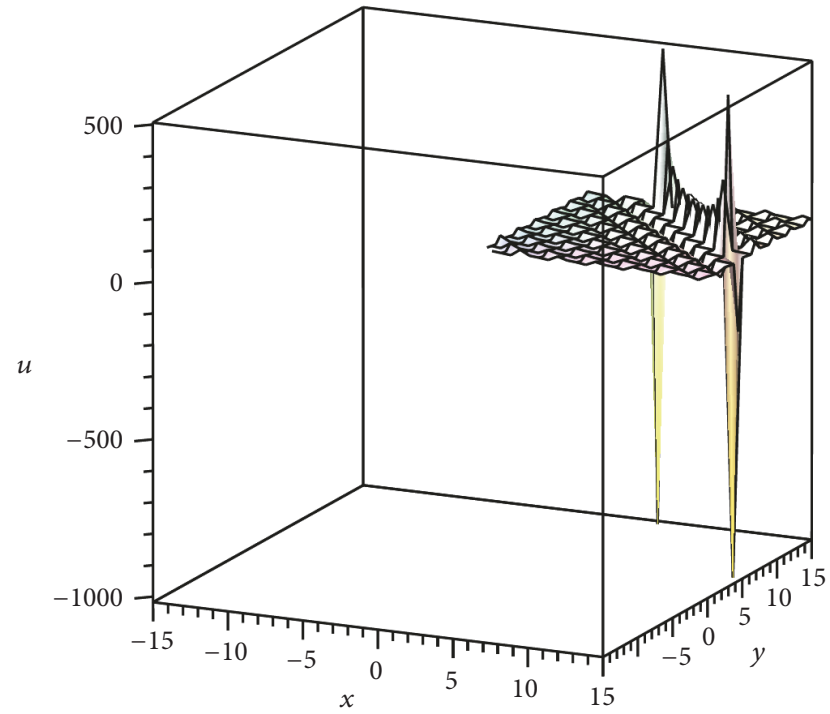

(b)

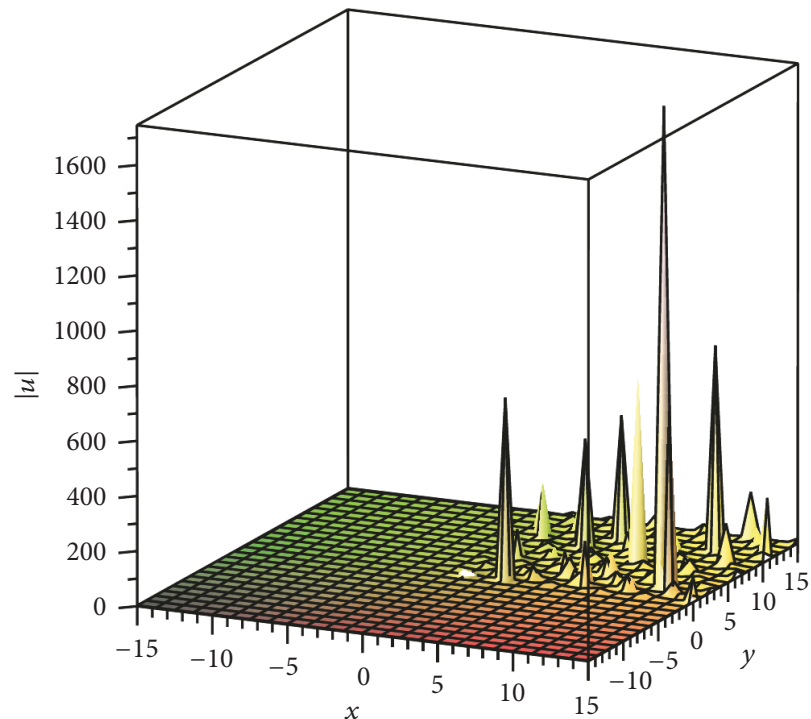

(d)

FIGURE 6: Associated plots of $u_{25}^{3}(x, y, z, t)$ in (124) with $-15 \leq x, y \leq 15$ using the novel $\left(G^{\prime} / G\right)$-expansion method: (a) singular periodic traveling wave solution for $u_{25}^{3}(x, y, z, t)$ with $\eta=\beta=1$, (b) singular double-soliton solution for $u_{25}^{3}(x, y, z, t)$ with $\eta=\beta=0.99$, (c) singular multiple-soliton solution for $u_{25}^{3}(x, y, z, t)$ with $\eta=0.9, \beta=0.8$, and (d) $\left|u_{25}^{3}(x, y, z, t)\right|$ with $\eta=0.9, \beta=0.8$.

solution, and a soliton solitary wave solution. It is observed that applying the novel $\left(G^{\prime} / G\right)$-expansion method to the equation has provided much more exact explicit solutions than the $\left(G^{\prime} / G, 1 / G\right)$-expansion method and other existing methods employed previously such as the $\left(G^{\prime} / G\right)$-expansion method. All of our results obtained by the methods have been constructed and verified by substituting them back into the original equation with the help of the Maple 17 program. In summary, the two methods with the aid of symbolic computation packages, used to solve the fractional JimboMiwa equation, are reliable, efficient, and convenient. Some of the obtained results are new and have not been attained previously. They may be of significant benefit for investigating some special traveling waves. The two methods could also be applied efficiently for a wide range of nonlinear fractional partial differential equations.

\section{Appendix}

\section{Solutions of the Generalized Riccati Equation}

Consider the following generalized Riccati equation:

$$
\phi^{\prime}(\xi)=r+p \phi(\xi)+q \phi^{2}(\xi),
$$

where $r, p$, and $q$ are variable real constants. In 2008, Zhu [60] provided twenty-seven exact solutions of (A.1) which can be 


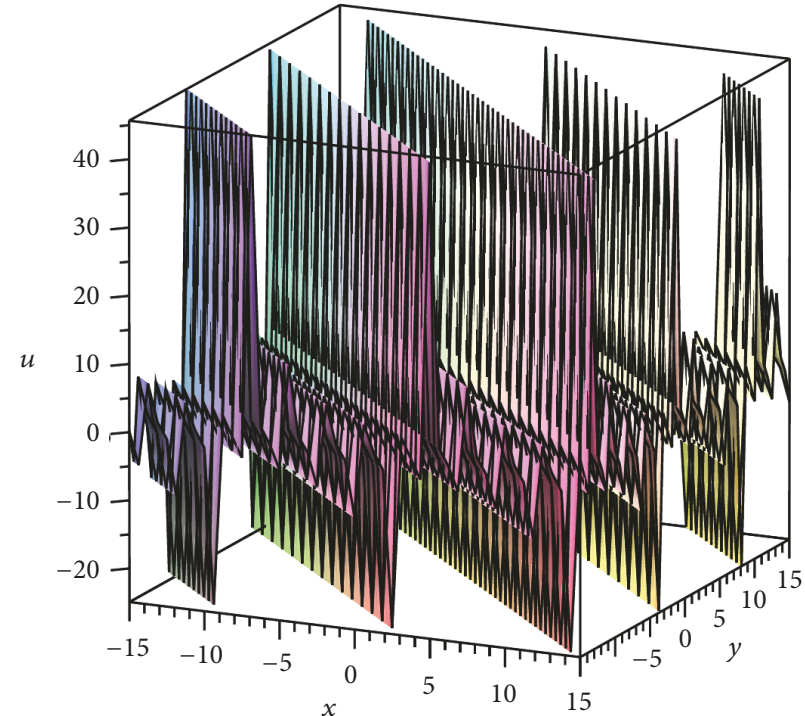

(a)

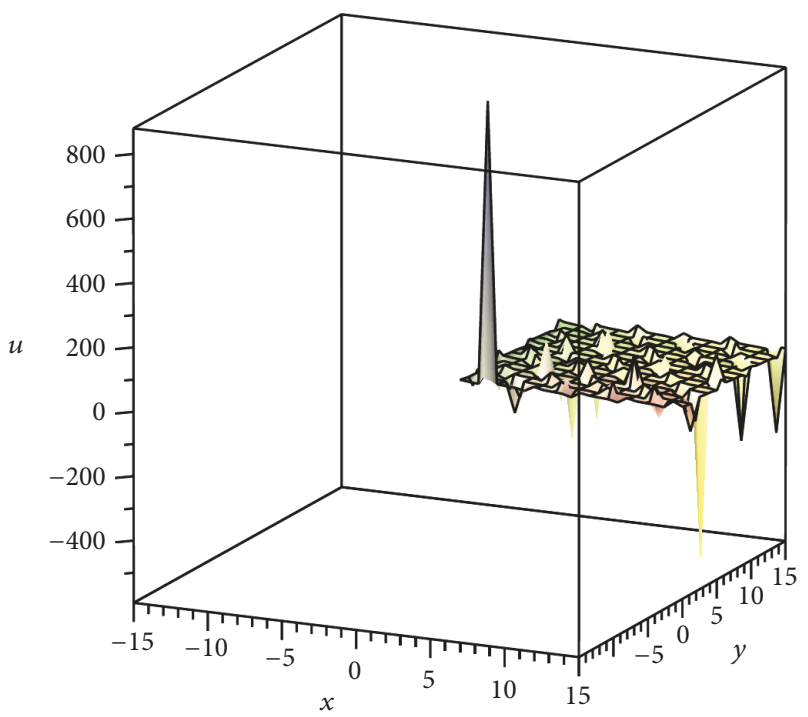

(c)

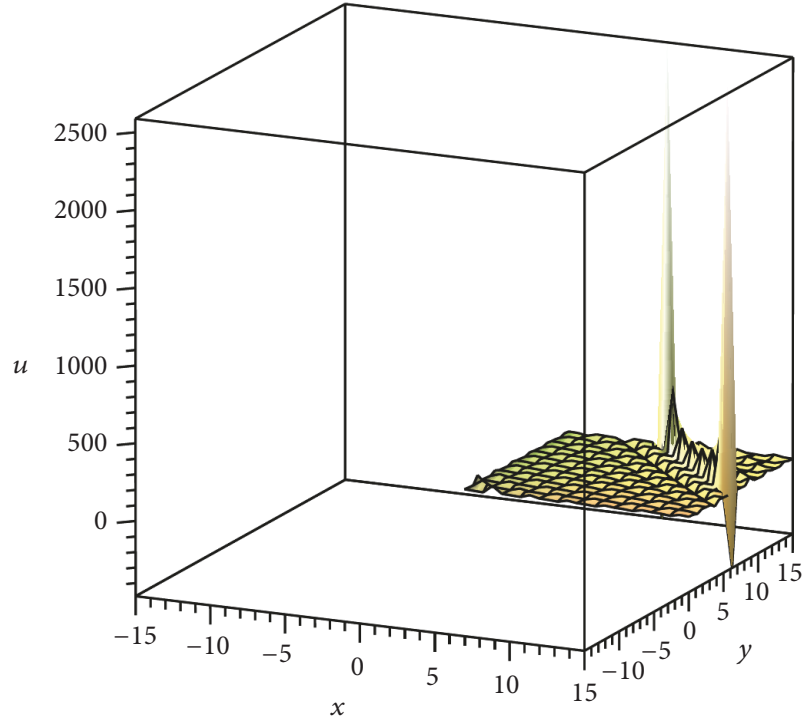

(b)

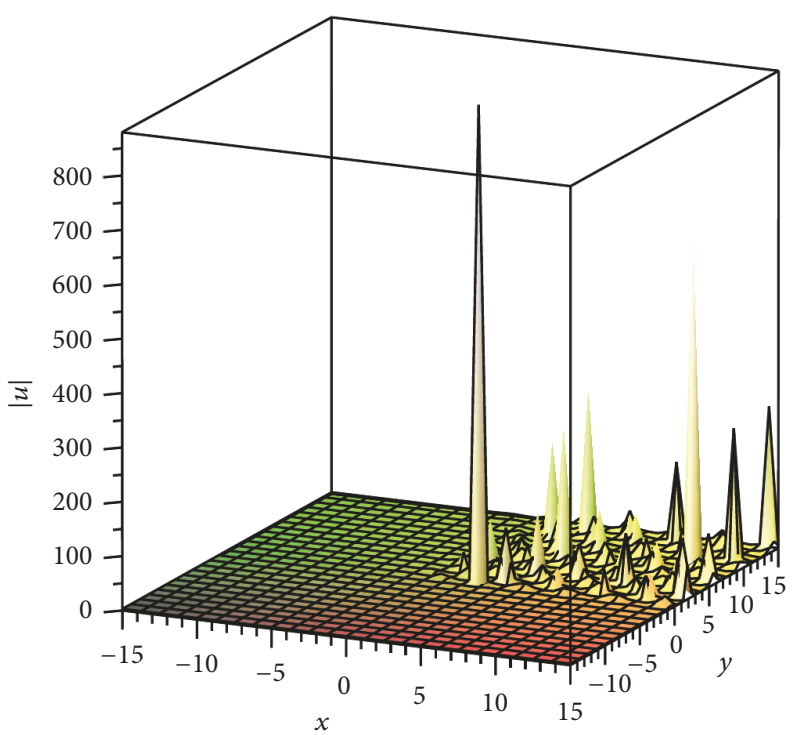

(d)

Figure 7: Associated plots of $u_{30}^{3}(x, y, z, t)$ in (127) with $-15 \leq x, y \leq 15$ using the novel $\left(G^{\prime} / G\right)$-expansion method: (a) periodic traveling wave solution for $u_{30}^{3}(x, y, z, t)$ with $\eta=\beta=1$, (b) singular double-soliton solution for $u_{30}^{3}(x, y, z, t)$ with $\eta=\beta=0.99$, (c) singular multiplesoliton solution for $u_{30}^{3}(x, y, z, t)$ with $\eta=0.9, \beta=0.8$, and (d) $\left|u_{30}^{3}(x, y, z, t)\right|$ with $\eta=0.9, \beta=0.8$.

used for the generalized Riccati equation mapping method. In 2009, Zheng [61] gave some comments on Zhu's paper that some of the provided solutions by Zhu are wrong from typewriting carelessness. However, we have here scrutinized which exact solutions provided by Zhu [60] are not a solution of (A.1). We have corrected the wrong ones, verifying the corrections by substitution back into (A.1) with the aid of the Maple 17 program. For the sake of easy identification, we have relabelled all of the solutions obtained by [60] and our corrections with our numbering system which are displayed as thirty-nine solutions. The corrected exact solutions of (A.1) are demonstrated below.

Family A.1. When $p^{2}-4 q r>0$ and $p q \neq 0$ (or $q r \neq 0$ ), the hyperbolic function solutions of (A.1) are as follows:

$$
\phi_{1}=-\frac{1}{2 q}\left[p+\sqrt{p^{2}-4 q r} \tanh \left(\frac{\sqrt{p^{2}-4 q r}}{2} \xi\right)\right] \text {, }
$$




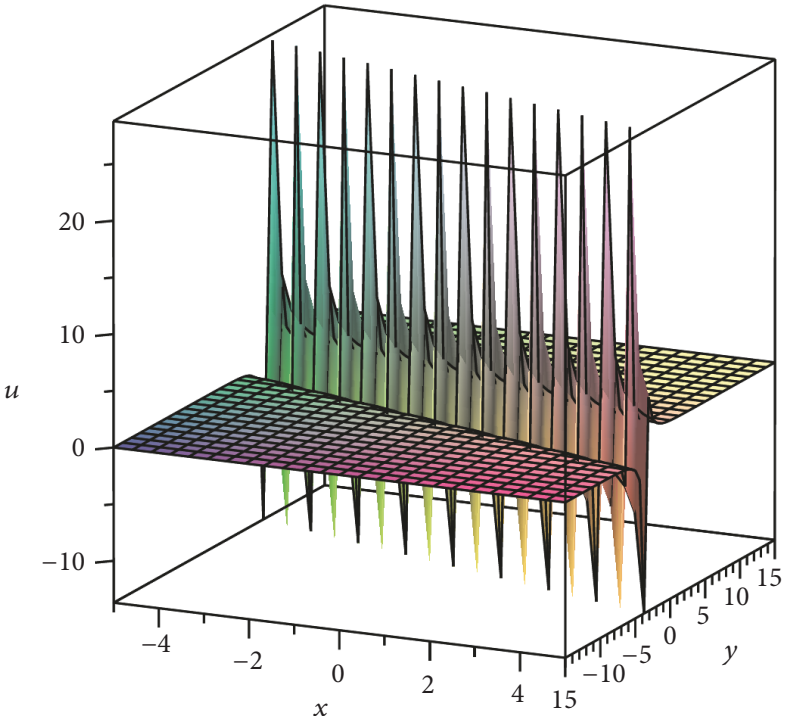

(a)

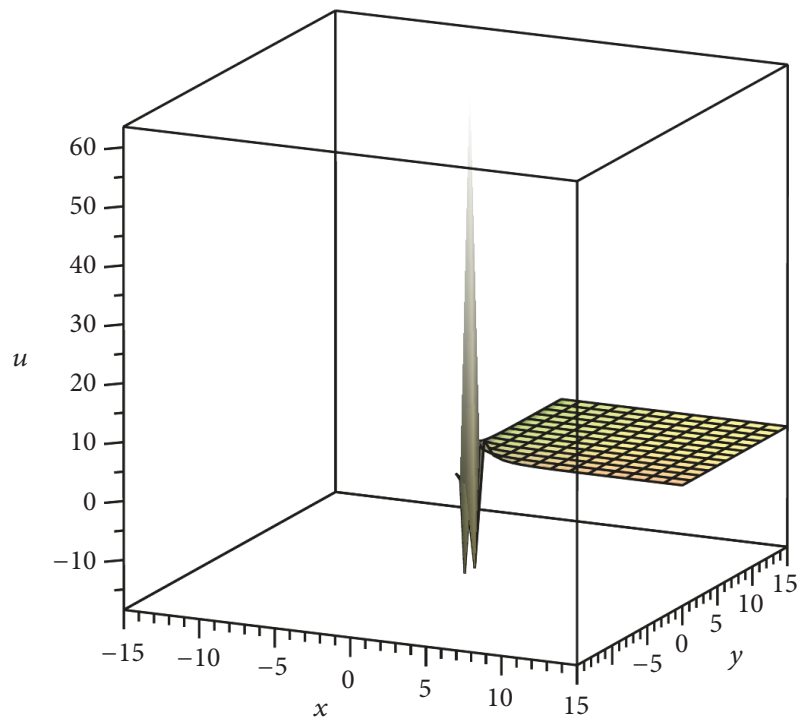

(c)

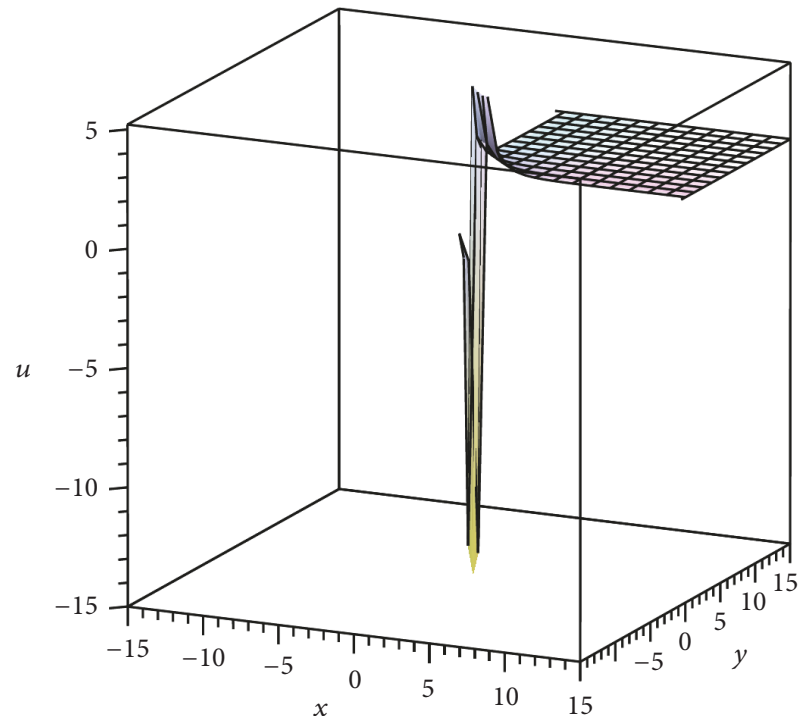

(b)

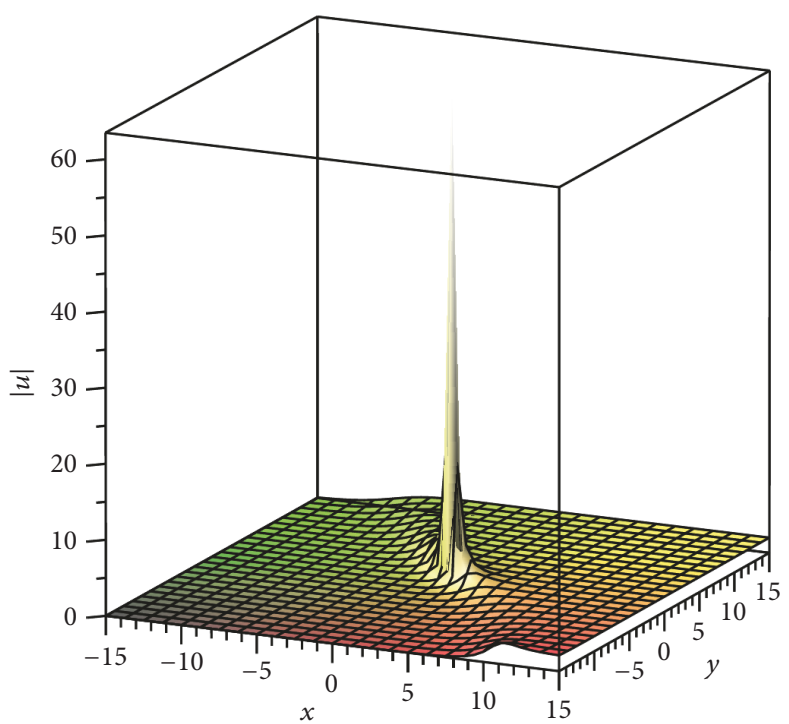

(d)

FIGURE 8: Associated plots of $u_{37}^{3}(x, y, z, t)$ in (132) with $-15 \leq x, y \leq 15$ using the novel $\left(G^{\prime} / G\right)$-expansion method: (a) solitary wave solution of singular kink type for $u_{37}^{3}(x, y, z, t)$ with $\eta=\beta=1$, (b) 1-soliton solitary wave solution of singular kink type for $u_{37}^{3}(x, y, z, t)$ with $\eta=\beta=0.99$, (c) 1-soliton solitary wave solution of singular kink type for $u_{37}^{3}(x, y, z, t)$ with $\eta=0.9, \beta=0.8$, and (d) $\left|u_{37}^{3}(x, y, z, t)\right|$ with $\eta=0.9, \beta=0.8$.

$$
\begin{aligned}
\phi_{2} & =-\frac{1}{2 q}\left[p+\sqrt{p^{2}-4 q r} \operatorname{coth}\left(\frac{\sqrt{p^{2}-4 q r}}{2} \xi\right)\right], \\
\phi_{3,4} & =-\frac{1}{2 q}\left[p+\sqrt{p^{2}-4 q r}\left(\tanh \left(\sqrt{p^{2}-4 q r} \xi\right) \pm i \operatorname{sech}\left(\sqrt{p^{2}-4 q r} \xi\right)\right)\right], \\
\phi_{5,6} & =-\frac{1}{2 q}\left[p+\sqrt{p^{2}-4 q r}\left(\operatorname{coth}\left(\sqrt{p^{2}-4 q r} \xi\right) \pm \operatorname{csch}\left(\sqrt{p^{2}-4 q r} \xi\right)\right)\right] \\
\phi_{7} & =-\frac{1}{4 q}\left[2 p+\sqrt{p^{2}-4 q r}\left(\tanh \left(\frac{\sqrt{p^{2}-4 q r}}{4} \xi\right)+\operatorname{coth}\left(\frac{\sqrt{p^{2}-4 q r}}{4} \xi\right)\right),\right.
\end{aligned}
$$




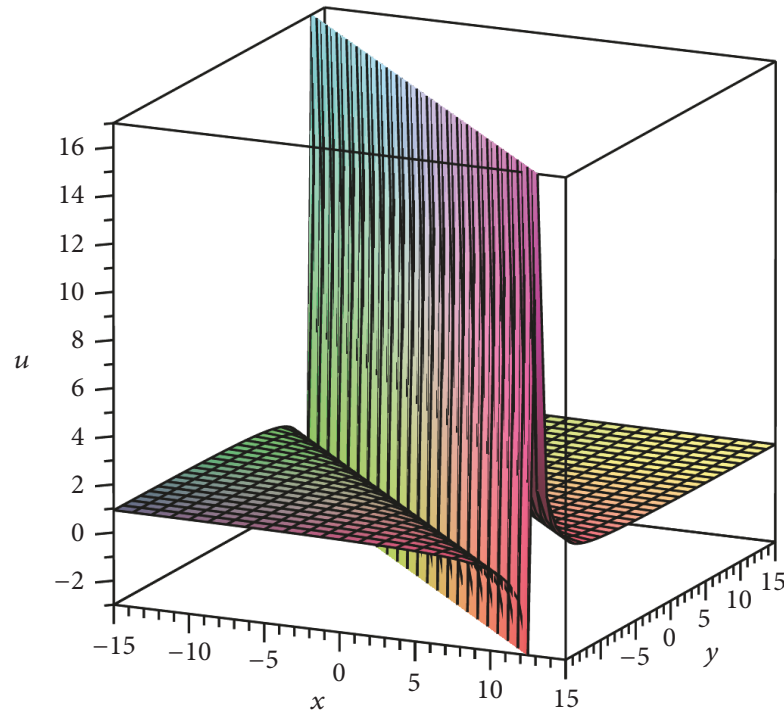

(a)

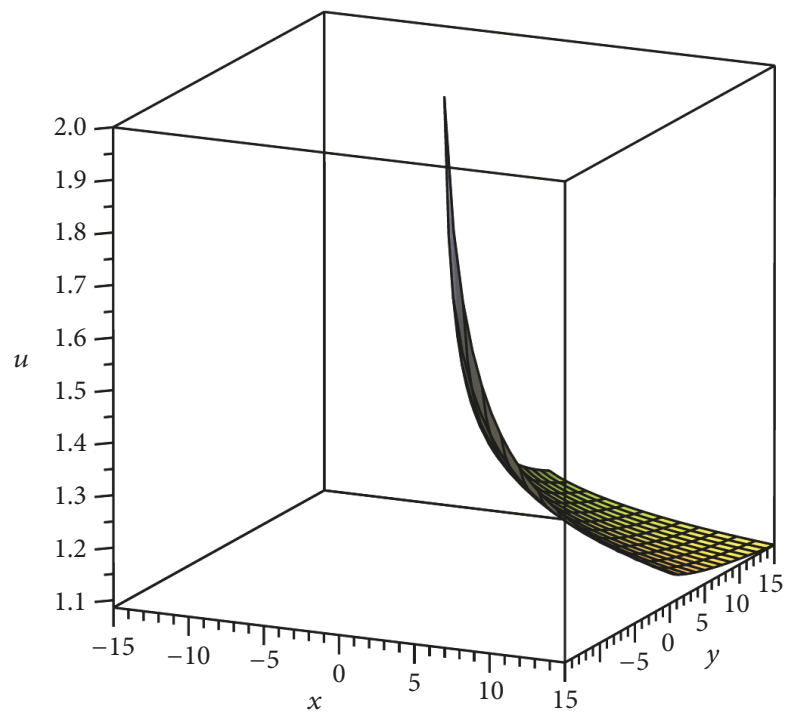

(c)

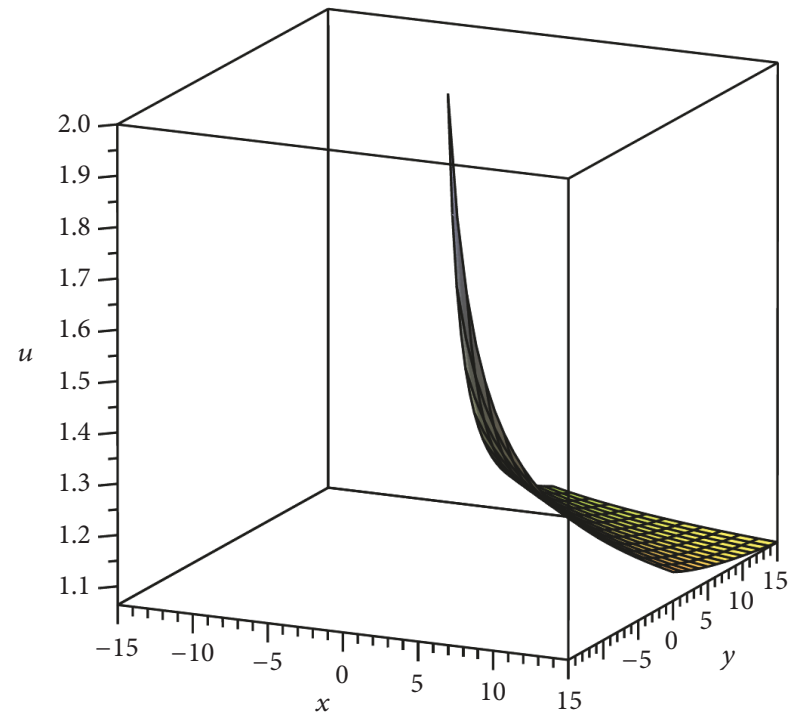

(b)

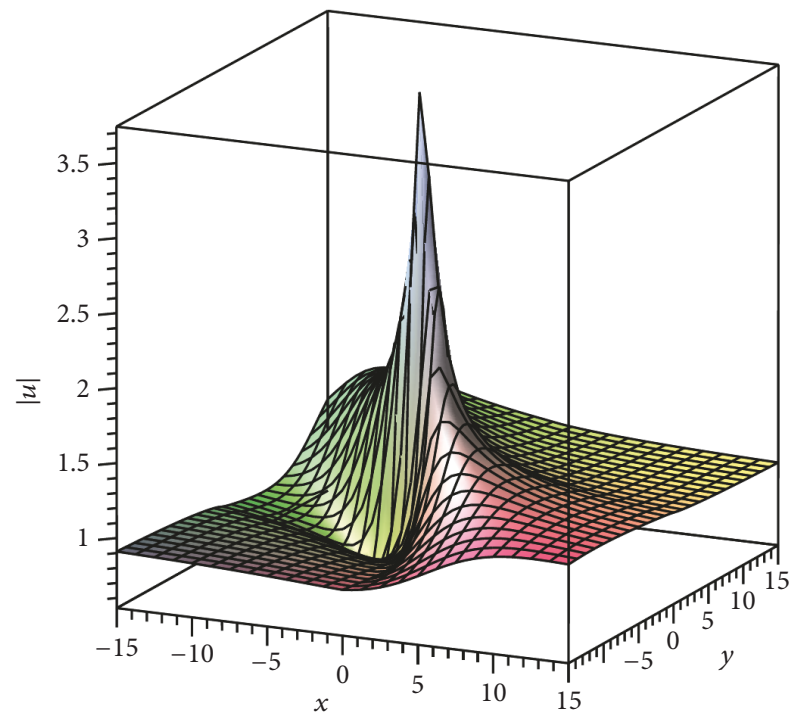

(d)

FIGURE 9: Associated plots of $u_{39}^{3}(x, y, z, t)$ in (134) with $-15 \leq x, y \leq 15$ using the novel $\left(G^{\prime} / G\right)$-expansion method: (a) solitary wave solution of singular kink type for $u_{39}^{3}(x, y, z, t)$ with $\eta=\beta=1$, (b) 1-soliton solitary wave solution for $u_{39}^{3}(x, y, z, t)$ with $\eta=\beta=0.99$, (c) 1 -soliton solitary wave solution for $u_{39}^{3}(x, y, z, t)$ with $\eta=0.9, \beta=0.8$, and $(\mathrm{d})\left|u_{39}^{3}(x, y, z, t)\right|$ with $\eta=0.9, \beta=0.8$.

$$
\begin{gathered}
\phi_{8,9}=\frac{1}{2 q}\left[-p+\frac{ \pm \sqrt{\left(A^{2}+B^{2}\right)\left(p^{2}-4 q r\right)}-A \sqrt{p^{2}-4 q r} \cosh \left(\sqrt{p^{2}-4 q r} \xi\right)}{A \sinh \left(\sqrt{p^{2}-4 q r} \xi\right)+B}\right], \\
\phi_{10,11}=\frac{1}{2 q}\left[-p-\frac{ \pm \sqrt{\left(B^{2}-A^{2}\right)\left(p^{2}-4 q r\right)}+A \sqrt{p^{2}-4 q r} \sinh \left(\sqrt{p^{2}-4 q r} \xi\right)}{A \cosh \left(\sqrt{p^{2}-4 q r} \xi\right)+B}\right],
\end{gathered}
$$

where $A$ and $B$ are two nonzero real constants that satisfy the condition $B^{2}-A^{2}>0$, 


$$
\begin{aligned}
& \phi_{12}=\frac{2 r \cosh \left(\left(\sqrt{p^{2}-4 q r} / 2\right) \xi\right)}{\sqrt{p^{2}-4 q r} \sinh \left(\left(\sqrt{p^{2}-4 q r} / 2\right) \xi\right)-p \cosh \left(\left(\sqrt{p^{2}-4 q r} / 2\right) \xi\right)}, \\
& \phi_{13}=\frac{-2 r \sinh \left(\left(\sqrt{p^{2}-4 q r} / 2\right) \xi\right)}{p \sinh \left(\left(\sqrt{p^{2}-4 q r} / 2\right) \xi\right)-\sqrt{p^{2}-4 q r} \cosh \left(\left(\sqrt{p^{2}-4 q r} / 2\right) \xi\right)}, \\
& \phi_{14,15}=\frac{2 r \cosh \left(\sqrt{p^{2}-4 q r} \xi\right)}{\sqrt{p^{2}-4 q r} \sinh \left(\sqrt{p^{2}-4 q r} \xi\right)-p \cosh \left(\sqrt{p^{2}-4 q r} \xi\right) \pm i \sqrt{p^{2}-4 q r}}, \\
& \phi_{16,17}=\frac{2 r \sinh \left(\sqrt{p^{2}-4 q r} \xi\right)}{-p \sinh \left(\sqrt{p^{2}-4 q r} \xi\right)+\sqrt{p^{2}-4 q r} \cosh \left(\sqrt{p^{2}-4 q r} \xi\right) \pm \sqrt{p^{2}-4 q r}},
\end{aligned}
$$

$\phi_{18}$

$$
=\frac{4 r \sinh \left(\left(\sqrt{p^{2}-4 q r} / 4\right) \xi\right) \cosh \left(\left(\sqrt{p^{2}-4 q r} / 4\right) \xi\right)}{-2 p \sinh \left(\left(\sqrt{p^{2}-4 q r} / 4\right) \xi\right) \cosh \left(\left(\sqrt{p^{2}-4 q r} / 4\right) \xi\right)+2 \sqrt{p^{2}-4 q r} \cosh ^{2}\left(\left(\sqrt{p^{2}-4 q r} / 4\right) \xi\right)-\sqrt{p^{2}-4 q r}} .
$$

Family A.2. When $p^{2}-4 q r<0$ and $p q \neq 0$ (or $q r \neq 0$ ), the trigonometric function solutions of (A.1) are as follows:

$$
\begin{aligned}
\phi_{19} & =\frac{1}{2 q}\left[-p+\sqrt{4 q r-p^{2}} \tan \left(\frac{\sqrt{4 q r-p^{2}}}{2} \xi\right)\right], \\
\phi_{20} & =-\frac{1}{2 q}\left[p+\sqrt{4 q r-p^{2}} \cot \left(\frac{\sqrt{4 q r-p^{2}}}{2} \xi\right)\right], \\
\phi_{21,22} & =\frac{1}{2 q}\left[-p+\sqrt{4 q r-p^{2}}\left(\tan \left(\sqrt{4 q r-p^{2}} \xi\right) \pm \sec \left(\sqrt{4 q r-p^{2}} \xi\right)\right)\right], \\
\phi_{23,24} & =-\frac{1}{2 q}\left[p+\sqrt{4 q r-p^{2}}\left(\cot \left(\sqrt{4 q r-p^{2}} \xi\right) \pm \csc \left(\sqrt{4 q r-p^{2}} \xi\right)\right)\right], \\
\phi_{25} & =\frac{1}{4 q}\left[-2 p+\sqrt{4 q r-p^{2}}\left(\tan \left(\frac{\sqrt{4 q r}-p^{2}}{4} \xi\right)-\cot \left(\frac{\sqrt{4 q r}-p^{2}}{4} \xi\right)\right]\right. \\
\phi_{26,27} & \left.=\frac{1}{2 q}\left[\begin{array}{c}
A \sin \left(\sqrt{4 q r-p^{2}} \xi\right)+B \\
\left.-A^{2}\right)\left(4 q r-p^{2}\right)
\end{array}\right)\right],
\end{aligned}
$$




$$
\phi_{28,29}=\frac{1}{2 q}\left[-p-\frac{ \pm \sqrt{\left(A^{2}-B^{2}\right)\left(4 q r-p^{2}\right)}+A \sqrt{4 q r-p^{2}} \cos \left(\sqrt{4 q r-p^{2}} \xi\right)}{A \sin \left(\sqrt{4 q r-p^{2}} \xi\right)+B}\right],
$$

where $A$ and $B$ are two nonzero real constants that satisfy the condition $A^{2}-B^{2}>0$,

$$
\begin{gathered}
\phi_{30}=-\frac{2 r \cos \left(\left(\sqrt{4 q r-p^{2}} / 2\right) \xi\right)}{\sqrt{4 q r-p^{2}} \sin \left(\left(\sqrt{4 q r-p^{2}} / 2\right) \xi\right)+p \cos \left(\left(\sqrt{4 q r-p^{2}} / 2\right) \xi\right)}, \\
\phi_{31}=\frac{2 r \sin \left(\left(\sqrt{4 q r-p^{2}} / 2\right) \xi\right)}{-p \sin \left(\left(\sqrt{4 q r-p^{2}} / 2\right) \xi\right)+\sqrt{4 q r-p^{2}} \cos \left(\left(\sqrt{4 q r-p^{2}} / 2\right) \xi\right)}, \\
\phi_{32,33}=-\frac{2 r \cos \left(\sqrt{4 q r-p^{2}} \xi\right)}{\sqrt{4 q r-p^{2}} \sin \left(\sqrt{4 q r-p^{2}} \xi\right)+p \cos \left(\sqrt{4 q r-p^{2}} \xi\right) \pm \sqrt{4 q r-p^{2}}}, \\
\phi_{34,35}=\frac{2 r \sin \left(\sqrt{4 q r-p^{2}} \xi\right)}{-p \sin \left(\sqrt{4 q r-p^{2}} \xi\right)+\sqrt{4 q r-p^{2}} \cos \left(\sqrt{4 q r-p^{2}} \xi\right) \pm \sqrt{4 q r-p^{2}}}, \\
\phi_{36}=\frac{4 r \sin \left(\left(\sqrt{4 q r-p^{2}} / 4\right) \xi\right) \cos \left(\left(\sqrt{4 q r-p^{2}} / 4\right) \xi\right)}{-2 p \sin \left(\left(\sqrt{4 q r-p^{2}} / 4\right) \xi\right) \cos \left(\left(\sqrt{4 q r-p^{2}} / 4\right) \xi\right)+2 \sqrt{4 q r-p^{2}} \cos ^{2}\left(\left(\sqrt{4 q r-p^{2}} / 4\right) \xi\right)-\sqrt{4 q r-p^{2}}} .
\end{gathered}
$$

Family A.3. When $r=0$ and $p q \neq 0$, the hyperbolic function solutions of (A.1) are as follows:

$$
\begin{aligned}
\phi_{37} & =\frac{-p c_{1}}{q c_{1}+\cosh (p \xi)-\sinh (p \xi)}, \\
\phi_{38} & =-\frac{p(\cosh (p \xi)+\sinh (p \xi))}{q\left(c_{1}+\cosh (p \xi)+\sinh (p \xi)\right)},
\end{aligned}
$$

where $c_{1}$ is an arbitrary constant.

Family A.4. When $q \neq 0$ and $r=p=0$, the rational function solution of (A.1) is as follows:

$$
\phi_{39}=-\frac{1}{q \xi+c_{2}}
$$

where $c_{2}$ is an arbitrary constant.

\section{Conflicts of Interest}

The authors declare that there are no conflicts of interest regarding the publication of this paper.

\section{References}

[1] D. Lu, A. Seadawy, and M. Arshad, "Applications of extended simple equation method on unstable nonlinear Schrödinger equations," Optik-International Journal for Light and Electron Optics, vol. 140, pp. 136-144, 2017.

[2] M. H. Bazyar and C. Song, "Analysis of transient wave scattering and its applications to site response analysis using the scaled boundary finite-element method," Soil Dynamics and Earthquake Engineering, vol. 98, pp. 191-205, 2017.

[3] S. Kumar, "A new fractional modeling arising in engineering sciences and its analytical approximate solution," Alexandria Engineering Journal, vol. 52, no. 4, pp. 813-819, 2013.

[4] V. F. Nikitin, N. N. Smirnov, M. N. Smirnova, and V. V. Tyurenkova, "On board electronic devices safety subject to high frequency electromagnetic radiation effects," Acta Astronautica, vol. 135, pp. 181-186, 2017.

[5] Siraj-ul-Islam and I. Ahmad, "Local meshless method for PDEs arising from models of wound healing," Applied Mathematical Modelling, vol. 48, pp. 688-710, 2017.

[6] Z. Qin, G. Mu, and H. Ma, “The $\left(G^{\prime} / G\right)$-expansion method for the fifth-order forms of KdV-Sawada-Kotera equation," Applied Mathematics and Computation, vol. 222, pp. 29-33, 2013. 
[7] D. Baldwin, Ü. Göktas, and W. Hereman, "Symbolic computation of hyperbolic tangent solutions for nonlinear differentialdifference equations," Computer Physics Communications, vol. 162, no. 3, pp. 203-217, 2004.

[8] E. H. Zahran and M. M. Khater, "Modified extended tanhfunction method and its applications to the Bogoyavlenskii equation," Applied Mathematical Modelling, vol. 40, no. 3, pp. 1769-1775, 2016.

[9] I. Aslan, "On the application of the exp-function method to the KP equation for $n$-soliton solutions," Applied Mathematics and Computation, vol. 219, no. 6, pp. 2825-2828, 2012.

[10] L. Ravi, S. Saha Ray, and S. Sahoo, "New exact solutions of coupled Boussinesq-Burgers equations by exp-function method," Journal of Ocean Engineering and Science, vol. 2, no. 1, pp. 3446, 2017.

[11] Y. Shi, Z. Dai, and D. Li, “The correct traveling wave solutions for the high-order dispersive nonlinear Schrödinger equation," Applied Mathematics and Computation, vol. 216, no. 5, pp. 15831591, 2010.

[12] A.-M. Wazwaz, “The Hirota's direct method and the tanh-coth method for multiple-soliton solutions of the Sawada-Kotera-Itö seventh-order equation," Applied Mathematics and Computation, vol. 199, no. 1, pp. 133-138, 2008.

[13] A.-M. Wazwaz, "The Hirota's direct method for multiple-soliton solutions for three model equations of shallow water waves," Applied Mathematics and Computation, vol. 201, no. 1-2, pp. 489-503, 2008.

[14] M. B. Hubert, G. Betchewe, S. Y. Doka, and K. Timoleon Crepin, "Soliton wave solutions for the nonlinear transmission line using the Kudryashov method and the $\left(G^{\prime} / G\right)$-expansion method," Applied Mathematics and Computation, vol. 239, pp. 299-309, 2014.

[15] P. N. Ryabov, D. I. Sinelshchikov, and M. B. Kochanov, "Application of the Kudryashov method for finding exact solutions of the high order nonlinear evolution equations," Applied Mathematics and Computation, vol. 218, no. 7, pp. 3965-3972, 2011.

[16] R. A. Van Gorder, "The variational iteration method is a special case of the homotopy analysis method," Applied Mathematics Letters, vol. 45, pp. 81-85, 2015.

[17] A.-M. Wazwaz, "The variational iteration method: A reliable analytic tool for solving linear and nonlinear wave equations," Computers and Mathematics with Applications, vol. 54, no. 78, pp. 926-932, 2007.

[18] I. Hashim, "Adomian decomposition method for solving BVPs for fourth-order integro-differential equations," Journal of Computational and Applied Mathematics, vol. 193, no. 2, pp. 658-664, 2006.

[19] K. C. Basak, P. C. Ray, and R. K. Bera, "Solution of non-linear Klein-Gordon equation with a quadratic non-linear term by Adomian decomposition method," Communications in Nonlinear Science and Numerical Simulation, vol. 14, no. 3, pp. 718-723, 2009.

[20] P. K. Bera and T. Sil, "Homotopy perturbation method in quantum mechanical problems," Applied Mathematics and Computation, vol. 219, no. 6, pp. 3272-3278, 2012.

[21] H. Saberi Nik, S. Effati, and M. Shirazian, "An approximateanalytical solution for the Hamilton-Jacobi-Bellman equation via homotopy perturbation method," Applied Mathematical Modelling, vol. 36, no. 11, pp. 5614-5623, 2012.
[22] L. Gavete, F. Ureña, J. J. Benito, A. García, M. Ureña, and E. Salete, "Solving second order non-linear elliptic partial differential equations using generalized finite difference method," Journal of Computational and Applied Mathematics, vol. 318, pp. 378-387, 2017.

[23] J. Jose, S.-J. Choi, K. E. T. Giljarhus, and O. T. Gudmestad, "A comparison of numerical simulations of breaking wave forces on a monopile structure using two different numerical models based on finite difference and finite volume methods," Ocean Engineering, vol. 137, pp. 78-88, 2017.

[24] M. Agrawal and C. S. Jog, "A quadratic time finite element method for nonlinear elastodynamics within the context of hybrid finite elements," Applied Mathematics and Computation, vol. 305, pp. 203-220, 2017.

[25] I. Podlubny, Fractional Differential Equations, vol. 198 of Mathematics in Science and Engineering, Academic Press, San Diego, Calif, USA, 1999.

[26] Q. Feng, "A new analytical method for seeking traveling wave solutions of space-time fractional partial differential equations arising in mathematical physics," Optik-International Journal for Light and Electron Optics, vol. 130, pp. 310-323, 2017.

[27] C. Huang, J. Cao, M. Xiao, A. Alsaedi, and F. E. Alsaadi, "Controlling bifurcation in a delayed fractional predator-prey system with incommensurate orders," Applied Mathematics and Computation, vol. 293, pp. 293-310, 2017.

[28] O. Marom and E. Momoniat, "A comparison of numerical solutions of fractional diffusion models in finance," Nonlinear Analysis. Real World Applications. An International Multidisciplinary Journal, vol. 10, no. 6, pp. 3435-3442, 2009.

[29] C. M. Pinto and A. R. Carvalho, "A latency fractional order model for HIV dynamics," Journal of Computational and Applied Mathematics, vol. 312, pp. 240-256, 2017.

[30] P. V. Ramana and B. K. R. Prasad, "Modified Adomian Decomposition Method for Van der Pol equations," International Journal of Non-Linear Mechanics, vol. 65, pp. 121-132, 2014.

[31] J.-S. Duan, T. Chaolu, and R. Rach, "Solutions of the initial value problem for nonlinear fractional ordinary differential equations by the Rach-Adomian-Meyers modified decomposition method," Applied Mathematics and Computation, vol. 218, no. 17, pp. 8370-8392, 2012.

[32] G.-C. Wu and D. Baleanu, "Variational iteration method for the Burgers' flow with fractional derivatives-new Lagrange multipliers," Applied Mathematical Modelling, vol. 37, no. 9, pp. 6183-6190, 2013.

[33] N. J. Ford, J. Xiao, and Y. Yan, "A finite element method for time fractional partial differential equations," Fractional Calculus and Applied Analysis. An International Journal for Theory and Applications, vol. 14, no. 3, pp. 454-474, 2011.

[34] C. A. Gómez S., "A nonlinear fractional Sharma-Tasso-Olver equation: new exact solutions," Applied Mathematics and Computation, vol. 266, pp. 385-389, 2015.

[35] E. A.-B. Abdel-Salam and G. F. Hassan, "Multi-wave solutions of the space-time fractional Burgers and Sharma-Tasso-Olver equations," Ain Shams Engineering Journal, vol. 7, no. 1, pp. 463$472,2016$.

[36] E. A.-B. Abdel-Salam and E. A. E. Gumma, "Analytical solution of nonlinear space-time fractional differential equations using the improved fractional Riccati expansion method," Ain Shams Engineering Journal, vol. 6, no. 2, pp. 613-620, 2015. 
[37] O. Guner, A. Bekir, and Ö. Ünsal, "Two reliable methods for solving the time fractional Clannish Random Walker's Parabolic equation," Optik-International Journal for Light and Electron Optics, vol. 127, no. 20, pp. 9571-9577, 2016.

[38] S. Demiray, Ö. Ünsal, and A. Bekir, "Exact solutions of nonlinear wave equations using $\left(G^{\prime} / G, 1 / G\right)$-expansion method," Journal of the Egyptian Mathematical Society, vol. 23, no. 1, pp. 78-84, 2015.

[39] E. M. E. Zayed and K. A. E. Alurrfi, "The $\left(G^{\prime} / G, 1 / G\right)$-expansion method and its applications to two nonlinear schrödinger equations describing the propagation of femtosecond pulses in nonlinear optical fibers," Optik-International Journal for Light and Electron Optics, vol. 127, no. 4, pp. 1581-1589, 2016.

[40] M. Shakeel and S. T. Mohyud-Din, "Soliton solutions for the positive Gardner-KP equation by $\left(G^{\prime} / G, 1 / G\right)$-expansion method," Ain Shams Engineering Journal, vol. 5, no. 3, pp. 951-958, 2014.

[41] L.-x. Li, E.-q. Li, and M.-1. Wang, "The $\left(G^{\prime} / G, 1 / G\right)$-expansion method and its application to travelling wave solutions of the Zakharov equations," Applied Mathematics. A Journal of Chinese Universities, vol. 25, no. 4, pp. 454-462, 2010.

[42] M. G. Hafez, M. N. Alam, and M. A. Akbar, "Exact traveling wave solutions to the Klein-Gordon equation using the novel $\left(G^{\prime} / G\right)$-expansion method," Results in Physics, vol. 4, pp. 177184, 2014.

[43] M. G. Hafez, "New travelling wave solutions of the $(1+1)$ dimensional cubic nonlinear Schrodinger equation using novel $\left(G^{\prime} / G\right)$-expansion method," Beni-Suef University Journal of Basic and Applied Sciences, vol. 5, no. 2, pp. 109-118, 2016.

[44] M. N. Alam, M. A. Akbar, and S. T. Mohyud-Din, "A novel $\left(G^{\prime} / G\right)$-expansion method and its application to the Boussinesq equation," Chinese Physics B, vol. 23, no. 2, pp. 20-203, 2014.

[45] M. N. Alam and M. A. Akbar, "Traveling wave solutions of the nonlinear $(1+1)$-dimensional modified Benjamin-BonaMahony equation by using novel $\left(G^{\prime} / G\right)$-expansion method," Physical Review \& Research International, vol. 4, pp. 147-165, 2014.

[46] M. Jimbo and T. Miwa, "Solitons and infinite-dimensional Lie algebras," Publications of the Research Institute for Mathematical Sciences, vol. 19, no. 3, pp. 943-1001, 1983.

[47] B. Cao, "Solutions of Jimbo-Miwa equation and Konopelchenko-Dubrovsky equations," Acta Applicandae Mathematicae, vol. 112, no. 2, pp. 181-203, 2010.

[48] A.-M. Wazwaz, "Multiple-soliton solutions for the CalogeroBogoyavlenskii-Schiff, Jimbo-Miwa and YTSF equations," Applied Mathematics and Computation, vol. 203, no. 2, pp. 592-597, 2008.

[49] Y. Shi and S. Han, "Solving Jimbo-Miwa equation by using the new test function method," in Proceedings of the 2011 7th International Conference on Natural Computation, ICNC 2011, pp. 2260-2263, July 2011.

[50] M. Mehdipoor and A. Neirameh, "New soliton solutions to the $(3+1)$-dimensional Jimbo-Miwa equation," Optik-International Journal for Light and Electron Optics, vol. 126, no. 23, pp. 4718-4722, 2015.

[51] G. Q. Xu, "The soliton solutions, dromions of the KadomtsevPetviashvili and Jimbo-Miwa equations in $(3+1)$-dimensions," Chaos, Solitons and Fractals, vol. 30, no. 1, pp. 71-76, 2006.
[52] W. Ma and J. Lee, "A transformed rational function method and exact solutions to the $(3+1)$ dimensional Jimbo-Miwa equation," Chaos, Solitons \& Fractals, vol. 42, no. 3, pp. 1356-1363, 2009.

[53] G. Jumarie, “Table of some basic fractional calculus formulae derived from a modified Riemann-Liouville derivative for non-differentiable functions," Applied Mathematics Letters. An International Journal of Rapid Publication, vol. 22, no. 3, pp. 378385, 2009.

[54] G. Jumarie, "On the derivative chain-rules in fractional calculus via fractional difference and their application to systems modelling," Central European Journal of Physics, vol. 11, no. 6, pp. 617-633, 2013.

[55] G. Jumarie, "Modified Riemann-Liouville derivative and fractional Taylor series of nondifferentiable functions further results," Computers \& Mathematics with Applications, vol. 51, no. 9, pp. 1367-1376, 2006.

[56] J.-H. He, S. K. Elagan, and Z. B. Li, "Geometrical explanation of the fractional complex transform and derivative chain rule for fractional calculus," Physics Letters. A, vol. 376, no. 4, pp. 257259, 2012.

[57] W.-H. Su, X.-J. Yang, H. Jafari, and D. Baleanu, "Fractional complex transform method for wave equations on Cantor sets within local fractional differential operator," Advances in Difference Equations, vol. 2013, no. 97, p. 8, 2013.

[58] O. Güner, A. Bekir, and A. C. Cevikel, "A variety of exact solutions for the time fractional Cahn-Allen equation," European Physical Journal Plus, vol. 130, no. 7, p. 146, 2015.

[59] B. Li, Y. Chen, and H. Zhang, "Explicit exact solutions for new general two-dimensional KDV-type and two-dimensional KDV-Burgers-type equations with nonlinear terms of any order," Journal of Physics A: Mathematical and General, vol. 35, no. 39, p. 8253, 2002.

[60] S. Zhu, "The generalizing Riccati equation mapping method in non-linear evolution equation: application to $(2+1)$-dimensional Boiti-Leon-Pempinelle equation," Chaos, Solitons and Fractals, vol. 37, no. 5, pp. 1335-1342, 2008.

[61] C.-L. Zheng, "Comments on 'The generalizing Riccati equation mapping method in nonlinear evolution equation: Application to $(2+1)$-dimensional Boiti-Leon-Pempinelle equation"', Chaos, Solitons and Fractals, vol. 39, no. 3, pp. 1493-1495, 2009.

[62] M. Wang, X. Li, and J. Zhang, “The $\left(G^{\prime} / G\right)$-expansion method and travelling wave solutions of nonlinear evolution equations in mathematical physics," Physics Letters A, vol. 372, no. 4, pp. 417-423, 2008.

[63] J. Zhang, F. Jiang, and X. Zhao, "An improved $\left(G^{\prime} / G\right)$-expansion method for solving nonlinear evolution equations," International Journal of Computer Mathematics, vol. 87, no. 8, pp. 17161725,2010

[64] M. A. Akbar, N. H. M. Ali, and E. M. E. Zayed, "A generalized and improved $\left(G^{\prime} / G\right)$-expansion method for nonlinear evolution equations," Mathematical Problems in Engineering, vol. 2012, Article ID 459879, p. 22, 2012.

[65] J. Zhang, X. Wei, and Y. Lu, "A generalized $\left(G^{\prime} / G\right)$-expansion method and its applications," Physics Letters A, vol. 372, no. 20, pp. 3653-3658, 2008.

[66] M. Kaplan and A. Bekir, "Construction of exact solutions to the space-time fractional differential equations via new approach," Optik-International Journal for Light and Electron Optics, vol. 132, pp. 1-8, 2017. 
[67] E. Aksoy, O. Guner, A. Bekir, and A. C. Cevikel, "Exact solutions of the $(3+1)$-dimensional space-time fractional Jimbo-Miwa equation," International Conference of Numerical Analysis and Applied Mathematics, vol. 1738, pp. 2900141-2900145, 2016.

[68] Z. Li and Z. Dai, "Abundant new exact solutions for the $(3+$ 1)-dimensional Jimbo-Miwa equation," Journal of Mathematical Analysis and Applications, vol. 361, no. 2, pp. 587-590, 2010. 


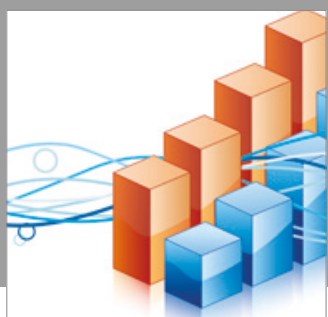

Advances in

Operations Research

vatersals

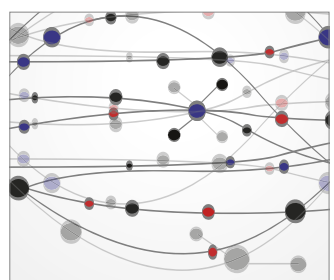

\section{The Scientific} World Journal
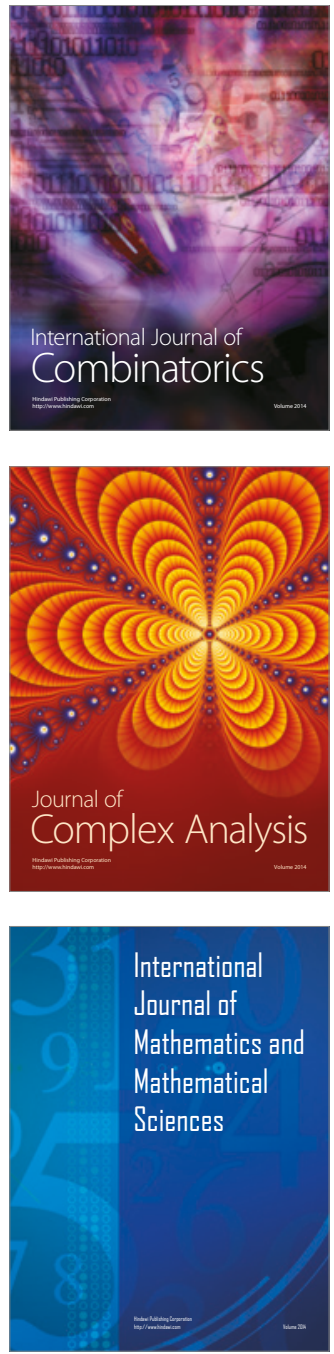
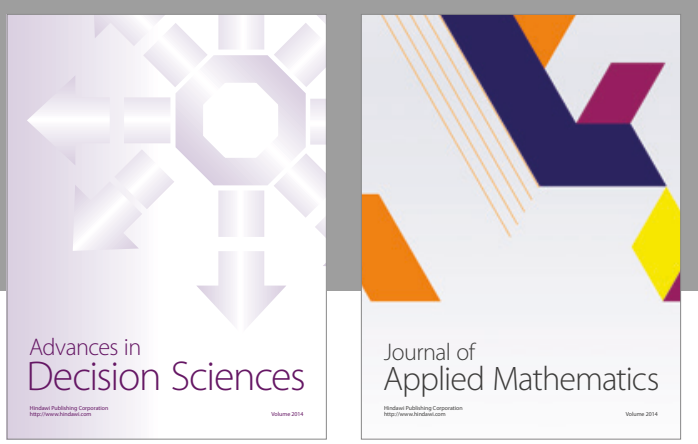

Algebra

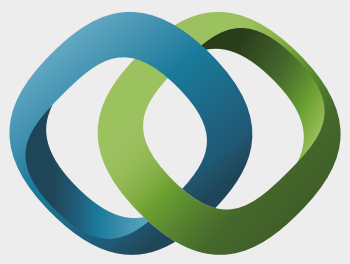

\section{Hindawi}

Submit your manuscripts at

https://www.hindawi.com
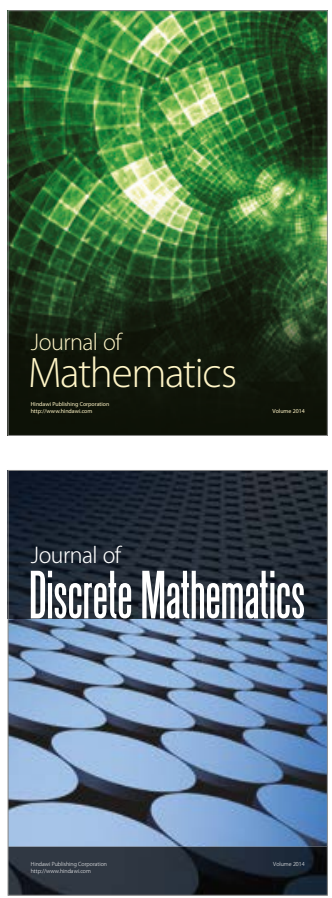

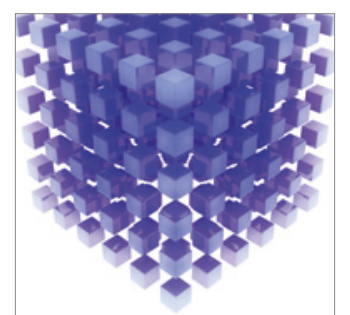

Mathematical Problems in Engineering
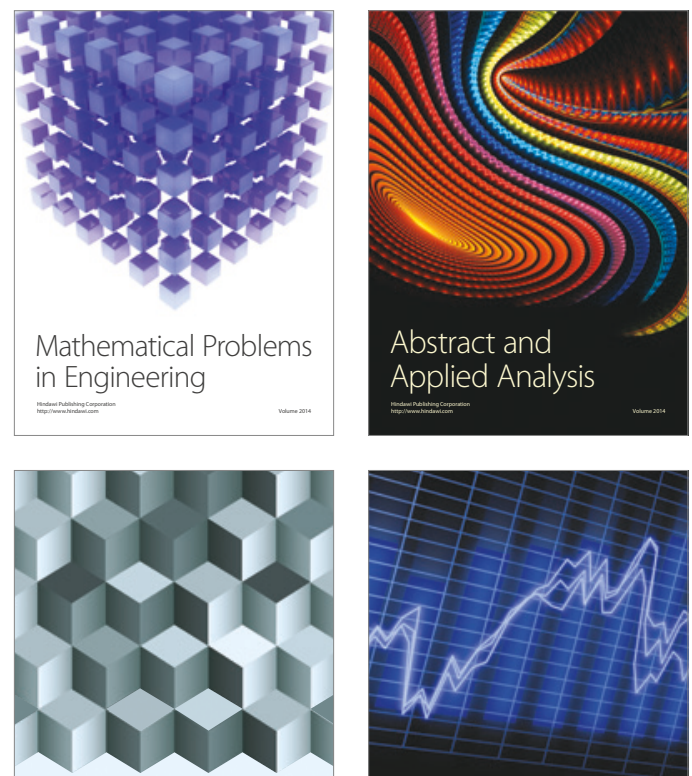

Journal of

Function Spaces

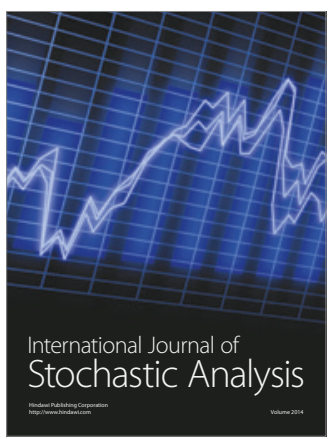

Probability and Statistics
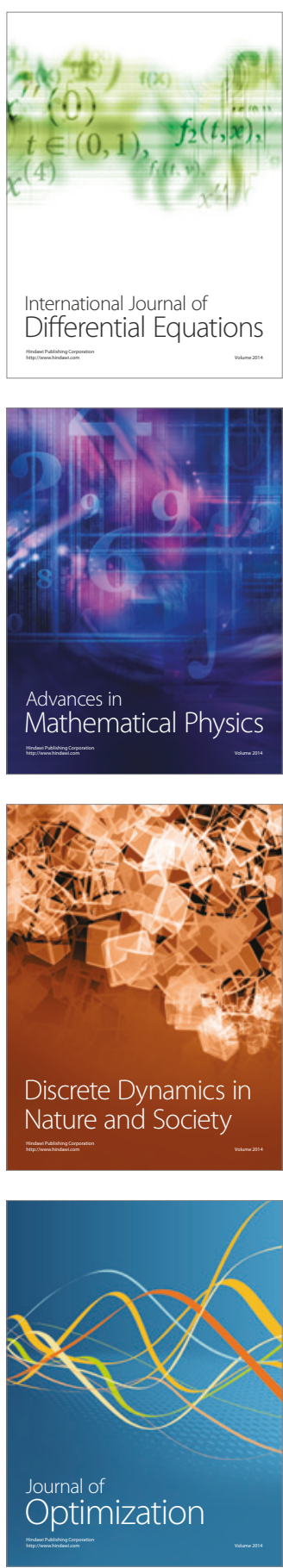